Der Medizinischen Fakultät der Georg-August-Universität Göttingen eingereicht von Prof. Dr. med. G. Zöller

\title{
Intraoperative Strahlenbelastung der Patienten bei der ureterorenoskopischen Steinentfernung
}

\author{
Inaugural-Dissertation \\ zur Erlangung des Doktorgrades \\ der Medizinischen Fakultät \\ der Georg-August-Universität zu Göttingen \\ vorgelegt von \\ Christiane Vowinkel
}

aus

Witzenhausen

Göttingen 2011 
Diese Dissertation wurde 2008 bis 2011 im Klinikum Bad Hersfeld unter Anleitung von Prof. Dr. med. G. Zöller geschrieben.

Dekan:

Prof. Dr. med. C. Frömmel

I. Berichterstatter:

Prof. Dr. med. G. Zöller

II. Berichterstatter/in:

Prof. Dr. rer. nat. P. Virsik-Köpp

III. Berichterstatter/in:

Tag der mündlichen Prüfung:

18.01 .2012 


\section{Inhaltsverzeichnis}

1. Einleitung 1

1.1 Therapie der Urolithiasis 3

1.1.1 Diagnostik bei Urolithiasis 3

1.1.2 Therapie der akuten Nierenkolik 6

1.1.3 Definitive Therapie bei Urolithiasis 6

$\begin{array}{lll}1.1 .4 & \text { Therapieleitlinien bei Urolithiasis } & 10\end{array}$

1.2 Strahlenbiologie und Strahlenschutz in der Medizin 12

$\begin{array}{ll}\text { 1.2.1 Strahlenexposition } & 12\end{array}$

1.2.2 Wirkung ionisierender Strahlung 12

1.2.3 Strahlenschutz in der Medizin 14

1.2.4 Strahlenexposition bei urologischen Untersuchungen 15

$\begin{array}{lll}1.3 & \text { Fragestellung } & 16\end{array}$

2. Material und Methode 19

$\begin{array}{lll}2.1 & \text { Patientenkollektiv } & 19\end{array}$

2.2 Röntgenarbeitsplatz und Dosisdokumentation 22

2.3 Ureterorenoskopische Steinentfernung 25

2.3.1 Prinzip der Ureterorenoskopie 25

2.3.2 Prinzip der ureterorenoskopischen Steinentfernung 31

2.4 Datenerhebung und statistische Methoden 37

3. Ergebnisse 38

3.1 Strahlenbelastung und Steinlokalisation 38

1.2 Strahlenbelastung und Form der Ureterorenoskopie 40

3.3 Strahlenbelastung und Steingröße 41

3.4 Strahlenbelastung und LASER-Lithotripsie 42

3.5 Strahlenbelastung und Durchleuchtungszeit 43

3.6 Strahlenbelastung und Anzahl der Röntgenaufnahmen 44

3.7 Strahlenbelastung und Einlage einer postoperativen Harnleiterschiene 47

$\begin{array}{lll}3.8 & \text { Strahlenbelastung und Operateure } & 48\end{array}$ 
4. Diskussion 53

5. Zusammenfassung 66

6. Literaturverzeichnis 68 


\section{Abkürzungsverzeichnis}

Abb.

ANOVA

AUA

BMI

Ca.

Charr.

cm

$\mathrm{cm}^{2}$

CT

DFP

DGU

DJ

EAU

ESWL

Fa.

FS

Gy

J

kg

kV
Abbildung

Varianzanalyse (englisch: analysis of variance)

American Urological Association

Body Mass Index

Zirka

Charrière

Zentimeter

Quadratzentimeter

Computertomographie

Dosisflächenprodukt

Deutsche Gesellschaft für Urologie

Doppel-J-Schiene

European Association of Urology

Extrakorporale Stoßwellenlithotripsie

Firma

Kraft in Wegrichtung

Gray, Einheit der Strahlendosis

Joule

Kilogramm

Kilovolt 
LASER

Light Amplification by Stimulated Emission of Radiation (Lichtverstärkung durch stimulierte Strahlenfreisetzung)

mSv

Millisievert

$\mathbf{m}^{2}$

Quadratmeter

$\mu G y^{*} m^{2}$

Einheit für das Dosis-Flächen-Produkt

$\mu \mathrm{m}$

Mikrometer

$\mathbf{m m}$

Millimeter

$\min$

Minute

MRT

Magnetresonanztomographie

p

Signifikanzwert (englisch: probability)

PCNL

PE

RöV

S

Sv

Tab.

TFT

URS

W

z.B.

Zum Beispiel 


\section{Einleitung}

Das Harnsteinleiden begleitet die Menschheit seit ihrem Beginn. Die ersten beschriebenen Harnsteine waren Steine der Harnblase, deren Behandlung bereits 500 Jahre vor Christi Geburt dokumentiert ist. Nach heutigen Erkenntnissen entstehen Harnsteine durch eine Störung des physikalisch-chemischen Gleichgewichtes des Harnes, wenn der Harn mit lithogenen Substanzen, die normalerweise mit dem Urin ausgeschieden werden, absolut oder relativ übersättigt ist. Die Löslichkeit lithogener Substanzen im Urin wird durch Komplexbildner erhöht, die das Ausfällen von Kristallen im Urin verhindern. Die wichtigsten Komplexbildner sind Zitrat und Magnesium, deren Mangel im Urin (Hypozitraturie bzw. Hypo-magnesurie) die Steinbildung fördert (Esen und Alken 2000, Fahlenkamp et al. 2008). Zusätzliche Faktoren wie Störungen im hydrodynamischen System der ableitenden Harnorgane, verminderte Flüssigkeitsaufnahme, Bewegungsarmut, hyperkalorische, ballaststoffarme Ernährung, Geschlecht (Männer erkranken deutlich häufiger an Harnsteinen), Alter und genetische Faktoren begünstigen die Steinbildung und können zur Bildung von unterschiedlich zusammengesetzten Harnsteinen führen. Die am häufigsten vorkommenden Steinarten sind Kalziumoxalatsteine (ca. 70 \%), Harnsäuresteine (ca. $15 \%$ ) und Struvitsteine (= Infektsteine, ca. $5 \%$ ). Seltene Steine wie Zystinund Xanthinsteine entstehen infolge von Stoffwechselstörungen. Häufig finden sich Mischsteine, die aus unterschiedlichen Komponenten bestehen (Schneider 1985). Der Anteil der Neuerkrankungen in der Gesamtbevölkerung (Inzidenz) beträgt ca. 1,47\%. Etwa 4,7\% der Menschen in Deutschland sind Steinträger (Prävalenz) (Hesse 2002). Die Wahrscheinlichkeit, einen zweiten oder weiteren Stein zu entwickeln, beträgt für unbehandelte Patienten ca. 60\% (Schneider 1982).

Die Harnsteinerkrankung stellte als eine der häufigsten urologischen Erkrankungen schon immer eine hohe Herausforderung dar. Dabei war es ein langer Weg von den fahrenden Steinschneidern des Mittelalters bis zu den heutigen minimal - invasiven endourologischen Operationen. 
In den letzten 20 Jahren haben technische Entwicklungen die Behandlung vor allen Dingen der Nieren- und Harnleitersteine revolutionär gewandelt. Seit Langem etablierte Operationsverfahren wurden von neuen Techniken, die unter dem Begriff der minimal-invasiven Therapien zusammenzufassen sind, abgelöst. Die Einführung der extrakorporalen Stoßwellenlithotripsie (ESWL), der minimalinvasiven, transurethralen und perkutanen Auxiliartechniken, der Lasertechnik und der modernen bildgebenden Verfahren waren Meilensteine auf dem Weg zu den heutigen Standardverfahren in der Steinbehandlung. Mit diesen Methoden lässt sich in einem hohen Maße eine Steinfreiheit erreichen. Ziel der heutigen klinischen Forschung ist deshalb, bei hoher Therapieeffektivität die Therapiemorbidität zu senken. Hierzu gehört auch die Frage der Strahlenbelastung bei den modernen Verfahren der Steintherapie. 


\subsection{Therapie der Urolithiasis}

\subsubsection{Diagnostik bei Urolithiasis}

Die Verdachtsdiagnose auf Vorliegen eines Harnsteinleidens ergibt sich aufgrund der typischen kolikartigen unilateralen Schmerzen. Die Diagnosestellung eines Harnsteinleidens und die Steinlokalisation erfolgen dann durch bildgebende Verfahren.

\section{Sonographie}

Die Ultraschalluntersuchung stellt die Basisdiagnostik in der Bildgebung dar. Steine werden aufgrund ihres charakteristischen Signalverhaltens - echoreicher Steinreflex mit dorsaler Schallauslöschung oder konsekutive Aufweitung des Nierenbeckenkelchssystems - erkannt. Hierbei beträgt die Sensitivität für einen Harnleiterstein 61 - 93 \%, die Spezifität 95 - 100 \%. (www.urologenportal.de 2009; Patlas et al. 2001).

\section{Konventionelle Abdomenleeraufnahme}

Die konventionelle Abdomenleeraufnahme kann einen Nieren- oder Harnleiterstein nachweisen, röntgennegative Steine entgehen jedoch dieser Diagnostik. Die Sensitivität wird mit bis zu 69 \%, die Spezifität mit bis zu 82 \% angegeben. (www.urologenportal.de 2009).

\section{$\underline{\text { Infusionsurogramm }}$}

Das Infusionsurogramm stellte bis vor Kurzem die Standarduntersuchung bei der Urolithiasis dar. Steine können aufgrund der kalkdichten Verschattung direkt oder indirekt als Kontrastmittelaussparung nach Kontrastmittelgabe (bei sogenannten röntgennegativen Steinen wie Harnsäuresteinen) lokalisiert werden. Die Kontrastmitteldarstellung des Hohlsystems erlaubt eine Übersicht über die vorliegenden topographischen Verhältnisse und erleichtert die Therapieplanung. Gegenüber der konventionellen Abdomenleeraufnahme erhöhen sich die Sensitivität auf 92 - $98 \%$ und die Spezifität auf $59-100 \%$. 


\section{$\underline{\text { Retrograde Pyelographie }}$}

Bei der retrograden Pyelographie erfolgt die Kontrastmittelgabe über einen Ureterenkatheter, der im Rahmen einer Zystoskopie retrograd in den Harnleiter vorgeschoben wird. Durch die direkte Applikation des Kontrastmittels in das Hohlsystem werden systemische Nebenwirkungen vermieden. Die retrograde Pyelographie ermöglicht damit die Kontrastmitteluntersuchung des oberen Harntrakts auch dann, wenn die intravenöse Kontrastmittelgabe wegen einer Nierenfunktionsstörung oder einer Kontrastmittelallergie kontraindiziert ist. In der Diagnostik des Harnsteinleidens hat diese Methode aber nach der Etablierung der Computertomographie in der Primärdiagnostik des Harnsteinleidens (siehe unten) an Bedeutung verloren. Unverzichtbar ist die retrograde Pyelographie allerdings bei der Harnleiterschienung, die bei therapieresistenten Schmerzen, beginnendem postrenalem Nierenversagen oder bei fieberhaftem Harnwegsinfekt notwendig wird. Zudem kann eine Harnleiterschienung elektiv in der Vorbereitung zu einer ureterorenoskopischen Steinentfernung eingelegt werden (siehe 2.3.1) (Strohmaier und Bartunek 2008).

\section{Computertomographie}

Die native Computertomographie gilt heute aufgrund ihrer hohen Sensitivität von $96-100 \%$ und hohen Spezifität von $92-100 \%$ als Standardverfahren zur Diagnosestellung bei Verdacht auf Urolithiasis (Abb.1) (Tamm et al. 2003).

\section{$\underline{\text { Kernspintomographie }}$}

Ein direkter Nachweis von Harnsteinen in der Kernspintomographie ist aufgrund des Prinzips der Kernspintomographie (Auslenkung von Wasserstoffionen im Magnetfeld) nicht möglich, da Harnsteine einen kristallinen Charakter ohne Wasserbeimengung aufweisen. In der Kernspintomographie können Steine im Harntrakt deshalb nur als Kontrastmittelaussparung nach Kontrastmittelgabe (Gadolinium) dargestellt werden.

Durch die Bildgebung werden sowohl Steinlokalisation, Steingröße und in gewissem Umfang auch die Steinzusammensetzung erkannt. Die Bildgebung erlaubt damit die Therapieplanung bei Urolithiasis. 


\section{Abbildung 1}

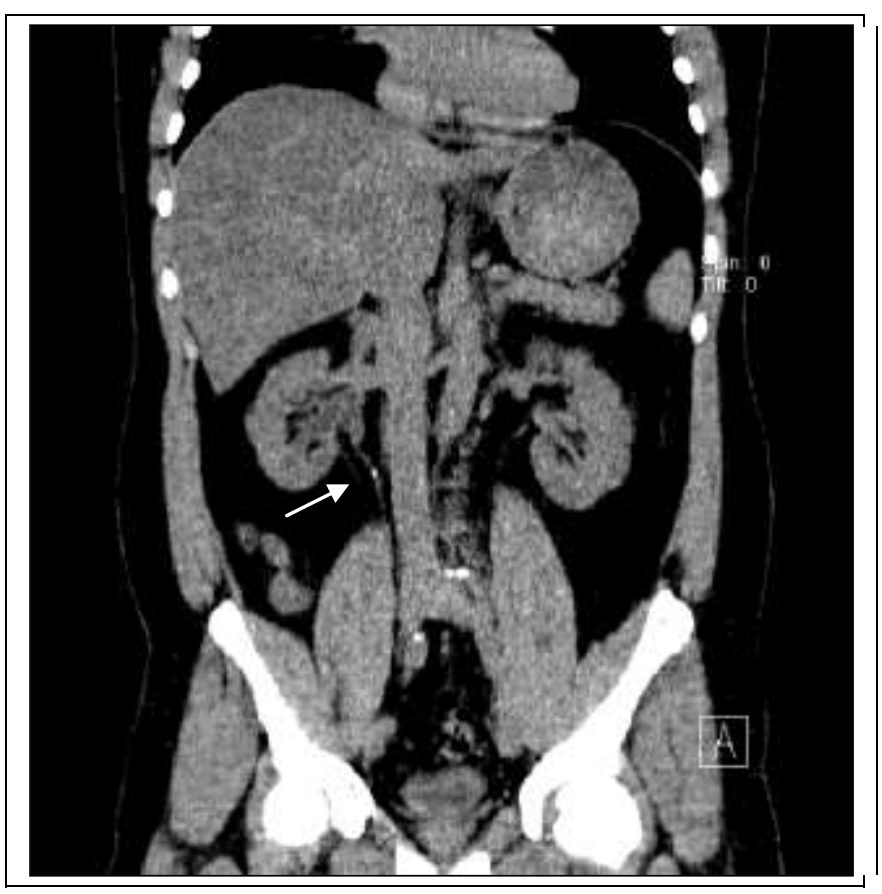

Native Computertomographie des Abdomens und des Beckens (ohne Kontrastmittelgabe) mit Nachweis eines proximalen Harnleitersteines (Pfeil) in der koronaren Bildrekonstruktion. 


\subsubsection{Therapie der akuten Nierenkolik}

Das charakteristische Symptom des Harnsteinleidens ist die "Steinkolik", ausgelöst durch einen Dehnungsschmerz des gestauten Hohlsystems bei obstruierend wirkendem Harnstein in einem Nierenkelch, im Nierenbeckenabgang oder im Harnleiter. In der akuten Phase einer "Steinkolik“ umfasst die Therapie zunächst die adäquate Schmerztherapie im Sinne eines Stufenkonzeptes mit nichtsteroidalen Antiphlogistika, Opiaten und Metamizol. Bei nicht ausreichender medikamentöser Schmerzbehandlung, bei Nierenstauung mit beginnenden Entzündungszeichen und/oder bei Anstieg der Nierenretentionswerte (Kreatinin) sowie bei Einzelnieren (Gefahr des postrenalen Nierenversagens) muss zwingend eine Entlastung der Niere durchgeführt werden. Dies geschieht entweder durch Einlage einer retrograd im Rahmen einer transurethralen Zystoskopie eingeführten Harnleiterschiene (DJ-Katheter) (Abb.2), oder, falls dies nicht möglich ist, alternativ durch einen perkutan eingelegten Nierenfistelkatheter (Nephrostomie) (Abb.3).

\subsubsection{Definitive Therapie bei Urolithiasis}

Nach der initialen Schmerztherapie, gegebenenfalls mit Sicherstellung des Harnabflusses durch Harnleiterschiene oder Nephrostomie, zielt die Therapie der postakuten Phase auf die definitive Steintherapie mit Entfernung des Nieren- bzw. Harnleitersteines.

Das Spektrum der Steintherapie umfasst dabei die konservative Therapie mit kontrolliertem Zuwarten und Abwarten eines spontanen Steinabganges sowie interventionelle Therapieformen wie die extrakorporale Stoßwellentherapie, die ureterorenoskopische Steinentfernung, die perkutane Steinentfernung und die offen-chirurgischen Steinoperationen.

Die Wahl des Therapieverfahrens wird durch die Größe, Lage und die vermutete Zusammensetzung des Steines sowie durch patientenspezifische Faktoren wie Konfiguration des Hohlsystems und Allgemeinzustand des Patienten bestimmt. 


\section{Abbildung 2}

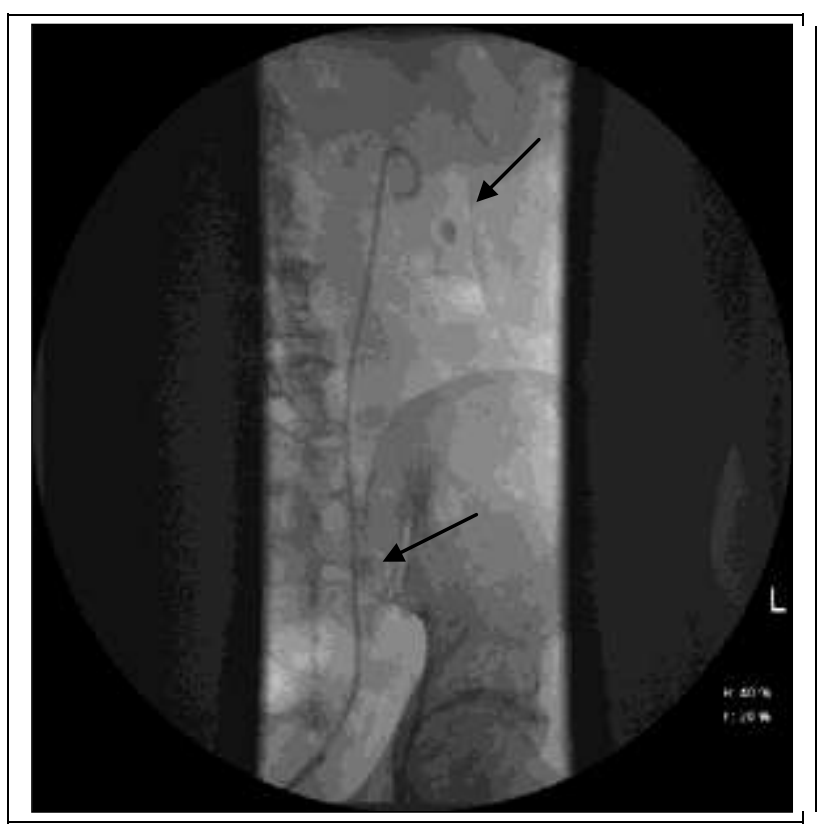

Ureterschiene (DJ-Katheter) bei distalem Harnleiterstein und unterem Nierenkelchstein (Pfeilmarkierungen).

\section{Abbildung 3}

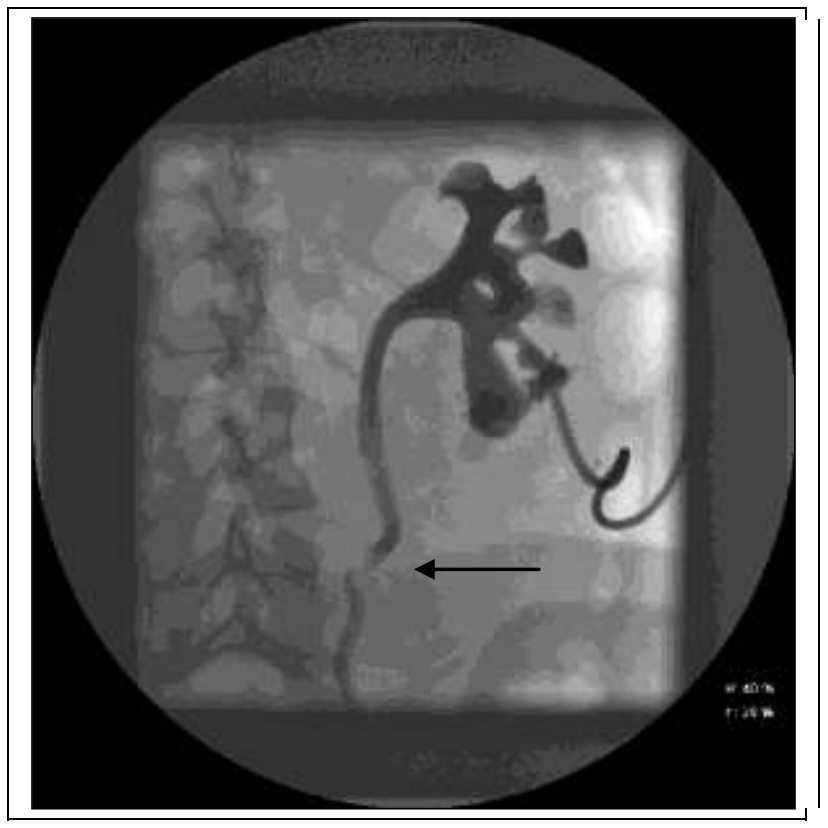

Perkutane Nephrostomie bei schwach schattengebendem obstruktivem Harnleiterstein (Pfeil). 


\section{$\underline{\text { Konservative Therapie }}$}

Die Wahrscheinlichkeit eines Spontanabganges liegt bei Harnleitersteinen unter einem Durchmesser von $5 \mathrm{~mm}$ bei über $50 \%$. Je distaler der Harnleiterstein liegt, umso größer ist diese Möglichkeit. Harnleitersteine mit einem Durchmesser von 6 - 10 mm können noch zu einem Prozentsatz von 25 - 53 \% spontan abgehen (Segura et al. 1997). Spontan abgangsfähige Steine in der Niere müssen bei Beschwerdefreiheit nicht therapiert werden. Auch bei etwas größeren Nierenkelchsteinen kann zunächst noch zugewartet werden. Eine Therapieindikation ergibt sich erst bei Auftreten von klinischen Beschwerden oder bei einer deutlichen Größenprogredienz. Die konservative Therapie besteht in der Gabe von reichlich Flüssigkeit in Kombination mit Bewegung, Gabe eines Antiphlogistikums und Gabe eines $\alpha$-1-Blockers bei distalen Harnleitersteinen. Auch Kalziumantagonisten und Nitrate scheinen nach aktueller Erfahrung den spontanen Steinabgang zu fördern (Türk et al. 2008). Bei symptomatischen Harnleitersteinen ist häufig zusätzlich eine Sicherung der Harnableitung mittels Harnleiterschienen erforderlich.

\section{Interventionelle Therapie}

Als interventionelle Therapie wird die aktive Entfernung der Steine aus dem Harntrakt oder die Zertrümmerung der Steine in abgangsfähige Fragmente bezeichnet.

- Extrakorporale Stoßwellenlithotripsie (ESWL)

ESWL-Geräte generieren auf piezoelektrischem, elektrohydraulischem oder elektromagnetischem Wege extrakorporal akustische Schockwellen. Die Schockwellen werden in einer akustischen Linse fokussiert und über einen Koppelbalg in den Körper des Patienten eingebracht. Dort entstehen an den akustischen Grenzflächen (Stein/Gewebe oder Stein/Urin) durch Impedanz starke Druck- und Zugspannungen, die zur Desintegration des Steins führen. Die Desintegrate müssen dann mit dem Urin ausgeschieden werden. 
Um die während der Zertrümmerung meist im Bereich der Ankopplungsstelle entstehenden Schmerzen zu kupieren bzw. zu therapieren, können lokale, orale oder intravenöse Analgetika eingesetzt werden (nicht steroidale Antiphlogistika wie Diclofenac oder Paracetamol, aber auch Opiate). Eine ESWL-Behandlung ist nahezu bei allen Steinen des oberen Harntraktes möglich, wenn der Stein sonografisch oder radiologisch geortet werden kann, wenn die Abgangsfähigkeit der Desintegrate gewährleistet ist und wenn keine sonstigen Kontraindikationen bestehen (www.urologenportal.de). Problematisch sind dabei Restkonkremente nach ESWL, die nicht ausgeschieden werden. Diese Restkonkremente stellen Kristallisationspunkte dar, an denen es zu einem erneuten Steinwachstum kommen kann. Kritisch sind insbesondere Steinlagen in der unteren Kelchgruppe. Hier ist in Abhängigkeit von der Steingröße mit einer Steinfreiheitsrate von 63 - 74 $\%$ bei einer Steingröße von über $10 \mathrm{~mm}$ und mit einer Steinfreiheitsrate von lediglich noch 14 - $33 \%$ bei einer Steingröße über $20 \mathrm{~mm}$ zu rechnen (Lingeman et al. 1995, Obek et al. 2001, Albala et al. 2001).

\section{- Ureterorenoskopische Steinentfernung}

Bei der ureterorenoskopischen Steinentfernung werden miniaturisierte Operationsendoskope retrograd über die Harnröhre und die Harnblase in den Harnleiter bzw. in das Nierenbeckenkelchsystem eingeführt. Nach endoskopischer Ortung des Steines können kleine Steine mittels Fasszangen oder Steinkörbchen (Dormiakörbchen) direkt entfernt werden, größere Steine müssen dagegen zunächst fragmentiert werden, bevor die Einzelfragmente geborgen werden können. Zur Steinfragmentierung (intrakorporale Lithotripsie) stehen unterschiedliche Verfahren wie die ballistische Stoßwellenlithotripsie, die piezoelektrische Stoßwellenlithotripsie oder als modernstes Verfahren die LASER-Lithotripsie zur Verfügung. Die ureterorenoskopische Steinentfernung wird ausführlich unter 2.3 Material und Methoden beschrieben.

- Perkutane Nephrolitholapaxie (PCNL)

Größere Steinmassen im Nierenbeckenkelchbereich können durch die retrograden endoskopischen Therapieverfahren oder durch die ESWL nicht ausreichend effektiv behandelt werden, da eine Steinfreiheit nicht erreicht werden kann. 
Effektivstes Therapieverfahren ist hier (neben einer offen-operativen Intervention siehe unten) die perkutane Nephrolitholapaxie. Hierbei wird in Intubationsnarkose in Bauchlage sonografisch gesteuert die Niere vorzugsweise über eine untere Kelchgruppe anpunktiert. Nach radiologischer Lagekontrolle mittels antegrader Pyelographie wird der Punktionstrakt über einen Führungsdraht in SeldingerTechnik aufbougiert. In der Standard-PCNL muss dabei ein Punktionstrakt von 26 - 30 Charr. etabliert werden. Gerätetechnische Weiterentwicklungen erlauben heute aber auch die sogenannte Mini-PCNL über einen Operationstrakt von 14 21 Charr. Über diesen Operationstrakt können dann verschiedene Lithotriptersysteme und Arbeitsgeräte wie flexible Endoskope in das Nierenbeckenkelchsystem eingebracht werden. Diese Geräte erlauben eine effektive Steinfragmentation und eine sichere Bergung der Steindesintegrate, so dass durch die PCNL auch bei großer Steinmasse in einem hohen Maße eine völlige Steinfreiheit erreicht werden kann. Zur Gewährleistung eines sicheren Harnabflusses erfolgt nach der Standard-PCNL die Einlage einer passageren Nephrostomie, nach der Mini-PCNL ist die Einlage einer passageren Ureterschiene ausreichend (www.urologenportal.de 2009).

\section{- Offen-operative Steinentfernung}

Offen-operative Steinentfernungen mittels Ureterolithotomie, Pyelolithotomie oder Nephrolithotomie werden heute kaum noch durchgeführt und kommen nur noch in Betracht, wenn simultan anatomische Pathologien wie z.B. eine Ureterabgangsenge operativ korrigiert werden müssen.

\subsubsection{Therapieleitlinien bei Urolithiasis}

Für die Behandlung von Harnsteinen wurden von den unterschiedlichen nationalen und internationalen urologischen Fachgesellschaften wie der Deutschen Gesellschaft für Urologie (DGU), der American Urologic Association (AUA) und der European Association of Urology (EAU) Leitlinien etabliert. Die Therapieleitlinien der DGU sind in Tabelle 1 zusammenfassend dargestellt (www.urologenportal.de). 


\section{Tabelle 1}

Leitlinien der Deutschen Gesellschaft für Urologie (DGU) zur Therapie der Urolithiasis (www.urologenportal.de).

\begin{tabular}{|c|c|c|c|}
\hline Steinlokalisation & Steingröße & $\begin{array}{c}\text { Therapie der } \\
\text { 1. Wahl }\end{array}$ & $\begin{array}{c}\text { Therapie der } \\
\text { 2. Wahl }\end{array}$ \\
\hline \multirow[t]{3}{*}{$\begin{array}{l}\text { Nierenbecken, obere und } \\
\text { mittlere Kelchgruppe }\end{array}$} & $<1 \mathrm{~cm}$ & $\begin{array}{c}\text { ESWL } \\
\text { Flexible URS }\end{array}$ & \\
\hline & $1-2 \mathrm{~cm}$ & ESWL & PCNL \\
\hline & $>2 \mathrm{~cm}$ & PCNL & ESWL \\
\hline \multirow[t]{3}{*}{ Untere Kelchgruppe } & $<1 \mathrm{~cm}$ & $\begin{array}{c}\text { ESWL } \\
\text { Flexible URS }\end{array}$ & \\
\hline & $1-2 \mathrm{~cm}$ & ESWL & PCNL \\
\hline & $>2 \mathrm{~cm}$ & PCNL & ESWL \\
\hline Nierenbeckenausgusssteine & & PCNL & $\begin{array}{c}\text { ESWL } \\
\text { Offene OP }\end{array}$ \\
\hline Proximaler Harnleiterstein & & ESWL & URS \\
\hline \multirow[t]{2}{*}{ Distaler Harnleiterstein } & $<1 \mathrm{~cm}$ & Konservativ & \\
\hline & $>1 \mathrm{~cm}$ & URS & ESWL \\
\hline
\end{tabular}




\subsection{Strahlenbiologie und Strahlenschutz in der Medizin}

\subsubsection{Strahlenexposition}

Menschen sind ständig einer natürlichen und einer künstlichen ionisierenden Strahlung ausgesetzt, die zu einer Strahlenexposition und somit auch zu einer Strahlenbelastung führt. Die natürliche Strahlenexposition, die sich aus terrestrischer und kosmischer Strahlung sowie aus der Strahlenbelastung durch Nahrungsaufnahme und durch Atmung (Radon) zusammensetzt, beträgt durchschnittlich 2,4 mSv/Jahr. Neben der natürlichen Strahlenexposition kommt es auch zur zivilisatorischen Strahlenbelastung. Sie wird verursacht durch Kernkraftwerke (maximale zulässige effektive Jahresdosis $=1 \mathrm{mSv}$ ) und durch medizinische Anwendung (Röntgendiagnostik, Strahlentherapie und Nuklearmedizin). Die zivilisatorische Strahlenexposition hat sich in den letzten Jahren deutlich erhöht. Sie wird vor allem durch die medizinischen Anwendungen bedingt. Spitzenreiter in der medizinischen Anwendung ist mit $90 \%$ die Röntgendiagnostik inklusive der interventionellen Radiologie, die Nuklearmedizin spielt mit ca. $4 \%$ ebenso wie die Strahlentherapie mit ca. $6 \%$ nur eine untergeordnete Rolle. Als Hauptursache der zunehmenden medizinischen Strahlenbelastung ist vor allem die stetig wachsende Anzahl der CT-Untersuchungen zu sehen, die im Vergleich mit konventionellen Röntgenaufnahmen mit einer höheren Strahlenbelastung assoziiert sind.

\subsubsection{Wirkung ionisierender Strahlung}

Strahlenwirkungen auf Zellen, Organe und ganze Menschen basieren überwiegend auf den direkten (Ionisationen) und indirekten (Wasserradiolyse und die Entstehung von reaktiven oxidativen Spezies) Strahlenwirkungen auf die DNA. Die DNA ist das Haupttarget. Geschädigte Lipide, RNAs und Proteine können mit Hilfe intakter DNA neu synthetisiert werden.

Betrachtet man die Strahlenwirkungen auf ganze Organe oder Menschen, kann man je nach der Strahlendosis zwischen deterministischen und stochastischen Strahlenwirkungen unterscheiden. 
Große Strahlendosen führen zur Schädigung bzw. Tötung vieler Zellen und damit zu deterministischen Wirkungen. Diese Wirkungen treten erst dann auf, wenn eine gewisse Mindestzahl an Zellen geschädigt oder getötet wird. Deshalb sind sie durch die Existenz eines Schwellenwertes charakterisiert, bei dessen Überschreiten eine deterministische Wirkung mit Sicherheit eintritt. Zu den bekannten deterministischen Effekten gehören z. B. die Augenlinsenkatarakt, die Strahlendermatitis, die Fibrose oder das Knochenmarks-Syndrom. Die Schwere der Erkrankung ist dosisabhängig.

Kleine Strahlendosen induzieren stochastische Wirkungen, also Wirkungen die mit einer, meist sehr kleinen Wahrscheinlichkeit auftreten. Zu den stochastischen Wirkungen gehören die genetischen Wirkungen (Wirkungen auf die Oozyten und Spermien, die sich erst in den nächsten Generationen manifestieren) und die somatischen Wirkungen (Wirkungen, die sich nach einer variablen Latenzzeit in der exponierten Person manifestieren, vor allem in Form einer Malignominduktion wie von Leukämien, von Lymphomen oder von solide Tumoren). Die Wahrscheinlichkeit einer Erkrankung nimmt linear mit der Dosis zu. Stochastische Wirkungen haben keinen Schwellenwert.

Um die Wirkung und Risiken einer Strahlenexposition auf biologisches Gewebe zu quantifizieren, werden unterschiedliche Größen verwendet, die mit Hilfe der Energiedosen in den einzelnen exponierten Organen berechnet werden (Shannoun et al. 2008).

Die Äquivalentdosis $\mathrm{H}$ wird berechnet als die Energiedosis $D(1 \mathrm{~J} / \mathrm{kg}=1 \mathrm{~Gy})$, die mit einem Strahlenwichtungsfaktor multipliziert wird, um den unterschiedlichen biologischen Wirkungen der unterschiedlichen Strahlenarten gerecht zu werden. Gamma- und Röntgenstrahlen als Referenzstrahlung haben eine Wichtungsfaktor von 1, Neutronen, Protonen und Alpha-Strahlung einen Wichtungsfaktor von 5 20. Die Einheit der Äquivalentdosis ist $1 \mathrm{~Sv}$. Die für ein bestimmtes Organ berechnete Äquivalentdosis (Organdosis) ermöglicht die Berechnung des jeweiligen Risikos.

Normalerweise führen röntgendiagnostische Untersuchungen nicht zu einer Ganzkörper- sondern nur zu partiellen Bestrahlungen. Für Strahlenschutzzwecke wird als Maß der Patientenbelastung die effektive Dosis, $D_{\text {eff, }}$ berechnet. 
Zur Berechnung der effektiven Dosis werden die einzelnen Organdosen mit Gewebewichtungsfaktoren multipliziert und aufsummiert. Die Gewebewichtungsfaktoren berücksichtigen die Organanteile und das unterschiedliche Krebsrisiko der verschiedenen Organe; bei Gonaden wird das genetische Risiko berücksichtigt. Die Einheit der effektiven Dosis ist ebenfalls 1 Sievert (Sv).

Die effektive Dosis kann nicht direkt gemessen werden. Sie kann aber in erster Näherung mit Hilfe des Dosis-Flächen-Produktes DFP (konventionelle Röntgendiagnostik) oder mit Hilfe des Dosis-Längen-Produktes DLP (Computertomografie) durch Multiplikation mit den entsprechenden untersuchungsspezifischen Konversionsfaktoren berechnet werden. Der untersuchungsspezifische Konversionsfaktor beträgt für Untersuchungen im Bereich des Abdomens 0,323.

Dabei wird das Dosis-Flächen-Produkt DFP $\left(\mathrm{cGy}^{*} \mathrm{~cm}^{2}\right.$ bzw. $\left.\mu \mathrm{Gy}^{*} \mathrm{~m}^{2}\right)$ von einem DFP-Messgerät (eine im Strahlengang des Röntgengerätes befindliche flache lonisationskammer) angezeigt. Das Dosis-Längen-Produkt DLP $\left(\mathrm{mGy}^{*} \mathrm{~cm}\right)$ wird

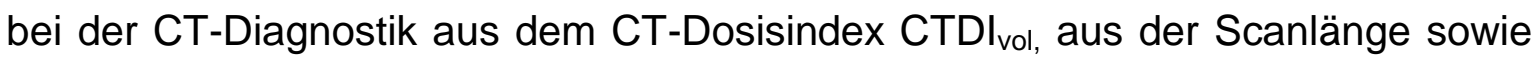
aus der Zahl der Rotationen durch Aufsummieren über die Zahl der Scanserien,

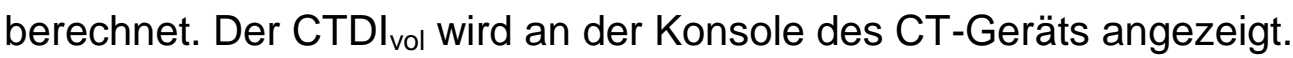

\subsubsection{Strahlenschutz in der Medizin}

Deterministische Schäden infolge diagnostischer Röntgenuntersuchungen sind wegen der geringen Strahlenbelastung in der Regel nicht zu erwarten. Dagegen können stochastische Wirkungen bei der diagnostischen Radiologie definitionsgemäß nicht ausgeschlossen werden. Zu diesen stochastischen Wirkungen zählen Veränderungen des Erbgutes und die strahleninduzierte Kanzerogenese.

Dieser Tatsache trägt die „Verordnung über den Schutz vor Schäden durch Röntgenstrahlen“ (RöV) Rechnung, die den optimalen Schutz Einzelner sowie der Allgemeinheit vor unnötigen und zu großen Röntgenstrahlung-Expositionen zum Ziel hat. Die neueste RöV in der Bundesrepublik Deutschland ist gültig seit dem 01.07.2002. Für jede Anwendung von Röntgenstrahlen muss von einem Arzt mit der entsprechenden Strahlenschutz-Fachkunde die rechtfertigende Indikation gestellt werden. Außerdem werden optimale Aufnahmebedingungen angestrebt, die durch die veröffentlichen Diagnostischen Referenzwerte vorgegeben werden. 
Dies wird durch eine korrekte Einstellungstechnik, durch einen einwandfreien Zustand der Geräte, durch eine korrekte Filmverarbeitung und durch eine auf das erforderliche Maß beschränkte Strahlenexposition gewährleistet (www.bmu.de 2002).

\subsubsection{Strahlenexposition bei urologischen Untersuchungen}

Wie in anderen Fachdisziplinen spielen auch in der urologischen Diagnostik röntgendiagnostische Untersuchungsmethoden eine herausragende Rolle. Die Strahlenbelastung der Patienten bei verschiedenen urologischen Untersuchungsmethoden ist in Tabelle 2 beispielhaft dargestellt. Bereits im Strahlenschutzbericht von 2004 wies das Bundesamt für Strahlenschutz darauf hin, dass die Strahlenbelastung in der Bundesrepublik Deutschland deutlich höher ist als in anderen europäischen Ländern. Im Jahr 1996 betrug die mittlere Anzahl der CT-Untersuchungen 0,06 / Einwohner, im Jahr 2006 betrug sie 0,11 / Einwohner (Bundesamt für Strahlenschutz). Sie nimmt weiterhin zu. Wie schon vorher erwähnt, ist in erster Linie der verstärkte Einsatz der Computertomographie für diese Entwicklung verantwortlich. Auch in der Urologie gewinnt dieses Verfahren bei der Steindiagnostik zunehmend an Bedeutung, da es bei der Diagnostik von Harnleitersteinen die höchste Sensitivität (95 - $100 \%$ ) und Spezifität (91 - 100\%) hat (Chowdhury et al. 2007, Knopfle et al. 2003).

\section{$\underline{\text { Tabelle } 2}$}

Beispiele für durchschnittliche effektive Dosis bei spezifischen radiologischen und nuklearmedizinischen Untersuchungsverfahren in der Urologie (Shannoun et al. 2008, Bundesamt für Strahlenschutz).

\begin{tabular}{|l|c|}
\hline Untersuchung & $D_{\text {eff }}(\mathrm{mSv})$ \\
\hline Nierenszintigramm & 0,8 \\
\hline Ausscheidungsurogramm & 2,5 \\
\hline Computertomographie (Abdomen/Becken) & 10 \\
\hline Nierenangiografie & 30 \\
\hline
\end{tabular}




\section{$1.3 \quad$ Fragestellung}

Unabhängig von den gültigen Leitlinien rücken bei der Therapiewahl zur Behandlung der Urolithiasis zunehmend die Aspekte von Therapieeffektivität und Therapiedauer in den Vordergrund. So wird heute in vielen Fällen von den Patienten ein konservativ abwartendes Verhalten aufgrund der dadurch entstehenden beruflichen Ausfallzeiten abgelehnt und eine zügige Therapie mit Erreichen einer Steinfreiheit gewünscht. Durch den Aspekt der erreichbaren Steinfreiheit wird auch die extrakorporale Stoßwellenlithotripsie (ESWL) als minimal invasives Therapieverfahren immer mehr in den Hintergrund gedrängt. Durch die ESWL kann zwar in 70 - 90 \% der Fälle eine Steindesintegration erreicht werden. Gehen aber diese Steinfragmente nicht spontan ab und verbleiben sie im Hohlsystem, so stellen sie Ausgangspunkte für ein erneutes Steinwachstum dar. Sind nach ca. 3 Monaten noch nicht alle Restkonkremente spontan abgegangen, so ist mit einer erneuten Ausbildung signifikanter Steine zu rechnen (Abb.4). Deshalb nimmt die Invasivität der Steintherapie in jüngster Zeit unter dem Gesichtspunkt der Steinfreiheit wieder zu. Insbesondere durch die technische Weiterentwicklung der semirigiden und flexiblen Ureterorenoskope kommt dieser Form der Steintherapie immer mehr Bedeutung zu. In der Klinik für Urologie und Kinderurologie des Klinikums Bad Hersfeld ist die Ureterorenoskopie in der Zwischenzeit Therapie der Wahl bei allen kleineren Steinen in Niere und Harnleiter. Dies spiegelt sich auch im Zahlenverhältnis der Steineingriffe wider.

So wurde an der Klinik für Urologie und Kinderurologie des Klinikum Bad Hersfeld zwischen dem 01.07.2008 und dem 01.07.2010 der überwiegende Anteil der Patienten mit Harnleiter- oder Nierensteinen mittels ureterorenoskopischer Steinentfernung behandelt (215 von 268 Patienten $=80,2 \%$ der Patienten). Zweithäufigster Eingriff war die perkutane Nephrolitholapaxie bei 45 Patienten (= $16,8 \%$ der Patienten). Die extrakorporale Stoßwellenlithotripsie kam nur noch bei 8 Patienten $(=3,0 \%$ der Patienten) zur Anwendung, offen operative Steinentfernungen wurden nicht durchgeführt (Tab.3). 


\section{Abbildung 4}

Signifikante Rezidivsteinbildung nach ESWL eines Nierenbeckenausgusssteines.

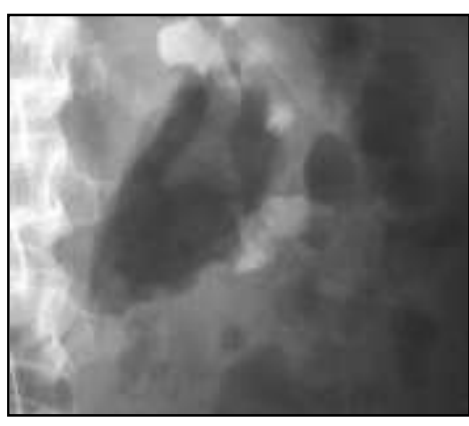

Prä-ESWL

Nierenbeckenausgussstein

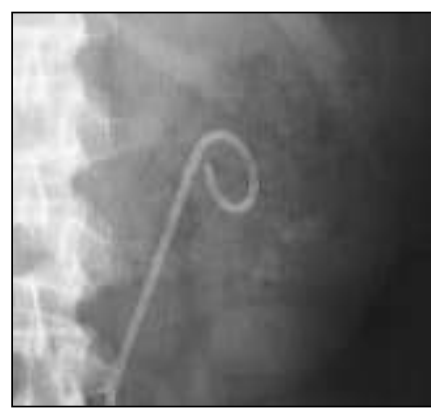

Post-ESWL mit Restkonkrementen

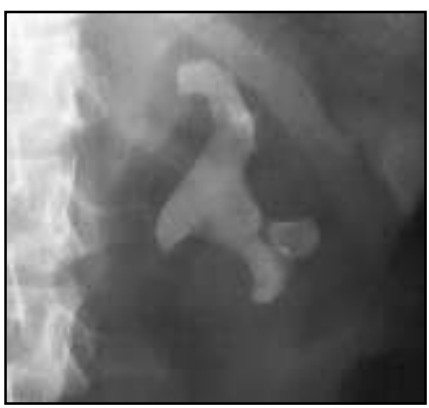

6 Jahre nach ESWL erneuter kompletter Nierenbeckenausgussstein.

\section{$\underline{\text { Tabelle } 3}$}

Steinbehandlungen in der Klinik für Urologie und Kinderurologie Bad Hersfeld im Untersuchungszeitraum 01.07.2008-01.07.2010.

\begin{tabular}{|l|r|}
\hline Extrakorporale Stoßwellenlithotripsie & 8 Patienten \\
\hline Ureterorenoskopische Steinentfernung & 215 Patienten \\
\hline Perkutane Nephrolitholapaxie & 45 Patienten \\
\hline Offen-operative Steinentfernung & 0 Patienten \\
\hline
\end{tabular}


Unter dem Aspekt der zunehmenden Verwendung der Ureterorenoskopie muss die Strahlenbelastung während dieses Eingriffes als mögliche Ursache einer therapieassoziierten Morbidität durch die stochastische Strahlenwirkung kritisch betrachtet werden. Aus diesem Grund werden in dieser Arbeit folgende Fragen hinsichtlich der Strahlenbelastung bei der Ureterorenoskopie untersucht:

Wie hoch ist die Strahlenbelastung bei der Ureterorenoskopie differenziert nach

1. Form der Ureterorenoskopie (semirigide versus flexible URS)?

2. Lage der Steine (Harnleiter versus Nierenbeckenkelchsystem)?

3. Größe der Steine bzw. daraus resultierender Art der Steinentfernung (einfache Steinextraktion versus LASER-Lithotripsie)?

4. Länge der Durchleuchtungszeit?

5. Anzahl der Röntgenaufnahmen zur Dokumentation während des Eingriffes?

6. Notwendigkeit der postoperativen Harnleiterschienung?

Darüber hinaus wird untersucht, in wie weit die gefundenen Ergebnisse sich bei unterschiedlichen Operateuren (mit und ohne Facharztstatus) unterscheiden, wie weit also die intraoperative Strahlenbelastung des Patienten auch von der individuellen Vorgehensweise unterschiedlicher Operateure beeinflusst wird. 


\section{Material und Methode}

\subsection{Patientenkollektiv}

Im Rahmen der Arbeit wurden alle Patienten erfasst, bei denen zwischen dem 01.07.2008 und dem 01.07.2010 eine Ureterorenoskopie zur Harnsteinbehandlung in der Klinik für Urologie und Kinderurologie des Klinikum Bad Hersfeld durchgeführt wurde. Der Startpunkt am 01.07.2008 wurde gewählt, da zu diesem Zeitpunkt der alte Röntgenarbeitsplatz der Klinik durch eine neue digitale Röntgeneinheit (Uroskop Access der Firma Siemens) ersetzt wurde (siehe 2.2. Röntgengerät und Dosismessung).

Bei den Patienten handelte es sich zum einen um Patienten, die mit einer akuten Nierenkolik notfallmäßig aufgenommen wurden, und bei denen im Verlauf wegen therapieresistenter Schmerzen, wegen persistierender Nierenstauung, wegen erhöhter Nierenretentionsparameter oder wegen ansteigender Entzündungswerte (Leukozytose) die Einlage einer Harnleiterschiene notwendig wurde. Zum anderen handelte es sich um Patienten, die von niedergelassenen Urologen gezielt zur Steintherapie zugewiesen wurden. Bei diesen Patienten wurde zur Vorbereitung der Ureterorenoskopie eine Harnleiterschiene eingelegt. Diese im Vorfeld eingelegte Harnleiterschiene führt zur Relaxation des Harnleiters und erleichtert damit wesentlich die Durchführung der Ureterorenoskopie.

Insgesamt wurden 215 Patienten behandelt. Die Patienten waren zwischen 17 und 88 Jahren alt, und das Durchschnittsalter betrug 53,3 Jahre. Frauen waren durchschnittlich etwas jünger als die Männer. Das Durchschnittsalter der Männer betrug 54,9 Jahre (18 - 88 Jahre), das der Frauen im Durchschnitt 49,4 Jahre (17 88 Jahre) (Abb.5). Männer waren mit einer Verteilung von 155:60 deutlich häufiger betroffen.

Es handelte sich sowohl um Patienten mit Harnleiter- als auch mit Nierensteinen. 70 Patienten hatten einen linksseitigen Harnleiterstein, 28 Patienten einen linksseitigen Nierenstein. Der rechte Harnleiter war bei 60 Patienten betroffen, die rechte Niere bei 33 Patienten. Steine sowohl im Harnleiter als auch in der Niere lagen bei 21 Patienten vor, bei 15 Patienten im linken Harntrakt, bei 6 Patienten im rechten Harntrakt. Drei Patienten hatten bilaterale Nierensteine (Tab.4). 


\section{Abbildung 5}

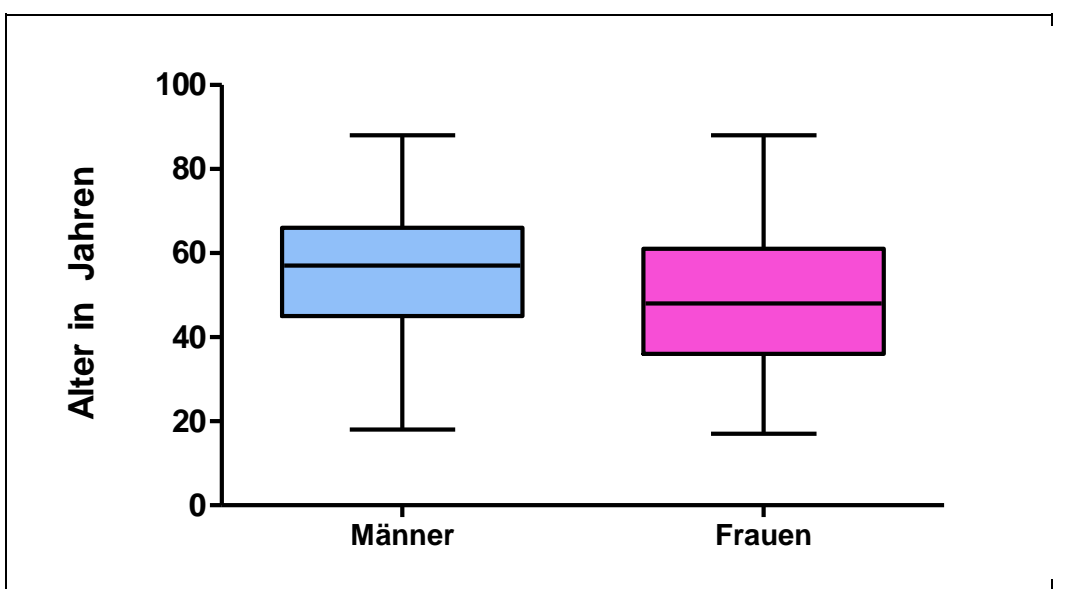

Altersverteilung der Patienten mit ureterorenoskopischer Steinentfernung.

$\underline{\text { Tabelle } 4}$

Steinlokalisation bei 215 behandelten Patienten.

\begin{tabular}{|l|c|c|c|}
\hline & Harnleiter & Niere & Harnleiter und Niere \\
\hline Rechtsseitig & 60 Patienten & 33 Patienten & 6 Patienten \\
\hline Linksseitig & 70 Patienten & 28 Patienten & 15 Patienten \\
\hline Beidseitig & 0 Patienten & 3 Patienten & 0 Patienten \\
\hline Zusammen & 130 Patienten & 64 Patienten & 21 Patienten \\
\hline
\end{tabular}


Die Anzahl der Steine pro Patient betrug zwischen einem und vier Steinen. Insgesamt wurden bei den 215 Patienten 305 Steine ureterorenoskopisch geborgen. Aus dem linken Harnleiter wurden 89 Steine entfernt, aus der linken Niere 65 Steine. 78 Steine wurden aus dem rechten Harnleiter geborgen, 62 Steine aus der rechten Niere. Die Zahl der bilateralen Nierensteine lag bei 11.

Die im Röntgenbild gemessene Steingröße variierte bei den Harnleitersteinen im maximalen Durchmesser zwischen 0,5 mm und 16,4 mm (im Mittel 6,3 mm) und bei den Nierensteinen zwischen $0,5 \mathrm{~mm}$ und $11,7 \mathrm{~mm}$ (im Mittel ebenfalls 6,3 $\mathrm{mm})$.

Bei allen Patienten erfolgte zunächst eine starre Ureterorenoskopie, bei 159 Patienten wurde zusätzlich eine flexible Ureterorenoskopie erforderlich. Bei 38 Patienten war eine primäre Steinbergung mittels Dormiakörbchen nicht möglich, so dass eine LASER-Lithotripsie zur Steindesintegration notwendig wurde. Bei 185 Patienten konnten die liegenden Ureterschienen im Rahmen der Ureterorenoskopie entfernt werden. Bei 30 Patienten wurden wegen komplexer intraoperativer Situationen wie Schleimhautläsionen oder Ureterwandperforationen erneut Ureterschienen eingelegt, die in der Regel ca. 7 Tage nach der Operation wieder entfernt werden konnten.

Insgesamt wurden die Operationen von drei erfahrenen Operateuren und zwei in der Ausbildung befindlichen Operateuren unter fachärztlicher Aufsicht durchgeführt. 


\subsection{Röntgenarbeitsplatz und Dosisdokumentation}

Die Ureterorenoskopie wird in der Regel unter röntgenologischer Bildwandlerkontrolle durchgeführt. Wichtige Operationsschritte werden dabei als Röntgenbild oder als Last-image-hold-Aufnahme des Bildwandlerbildes dokumentiert. Dabei bieten die Röntgenbilder eine sehr gute Bildauflösung. Die Röntgenaufnahme bedeutet jedoch für den Patienten eine zusätzliche Strahlenbelastung. Die Lastimage-hold-Aufnahme speichert das letzte Durchleuchtungsbild ohne zusätzliche Strahlenbelastung, allerdings ist die Bildauflösung der Last-image-hold-Aufnahme deutlich schlechter (Abb.6).

In der Klinik für Urologie und Kinderurologie Bad Hersfeld steht seit dem 01.07.2008 das Uroskop Access der Fa. Siemens, Erlangen, Deutschland, als Röntgenarbeitsplatz zur Verfügung. Das Uroskop Access besteht aus einem Obertischröntgenstrahler und einer Patientenlagerungsplatte, die einen freien Zugang zum Patienten und eine adäquate Lagerung des Patienten (z. B. modifizierte Steinschnittlagerung bei der Ureterorenoskopie) erlaubt (Abb.7). Das Röntgengerät und die motorgetriebene Tischbewegung können über eine Bedienkonsole am Röntgengerät selbst, eine Bedienkonsole an einem im Raum befindlichen Schreibtisch sowie für die intraoperative Bedienung über einen Multifunktions-Fußschalter gesteuert werden.

Als Röntgengenerator ist in das Uroskop Access-System ein HochfrequenzRöntgengenerator POLYDOROS SX 65/80 mit Belichtungsautomatik integriert. Für die Durchleuchtung und für die Röntgenaufnahme sind dabei angepasst an die verschiedenen diagnostischen und therapeutischen radiologischen Verfahren in der Urologie unterschiedliche Betriebsarten vorprogrammiert (Organprogramme). Die Ureterorenoskopie erfolgt dabei mit dem Organprogramm „Retrograde Pyelographie“. Dieses Organprogramm beinhaltet eine Röhrenspannung von $79 \mathrm{kV}$, einen Kupferfilter für die Röntgendurchleuchtung von 0,2 $\mathrm{mm}$ und für die Röntgenaufnahme von 0,1 $\mathrm{mm}$. Bei der Durchleuchtung werden dabei 25 Bilder/Sekunde erzeugt. Die Bildeinblendung mittels Irisblenden erfolgt manuell an der Bedienungskonsole am Röntgenstativ. Der Fokusabstand beträgt standardmäßig $115 \mathrm{~cm}$. 
Abbildung 6
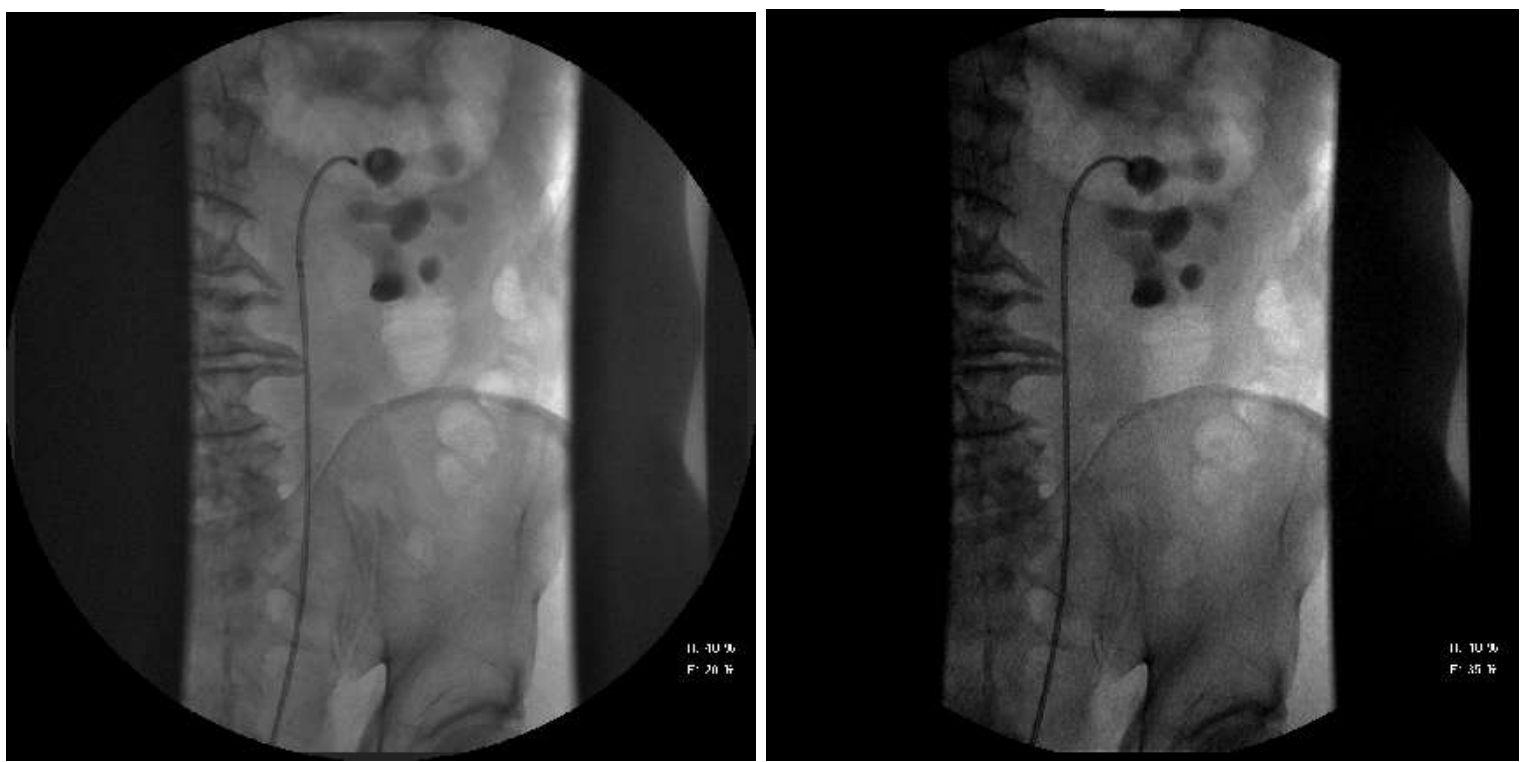

Konventionelle Röntgenaufnahme (links) und Last-image-hold-Aufnahme (rechts) des gleichen Motivs während einer flexiblen Ureterorenoskopie mit Kontrastmittelaussparung im oberen Nierenkelch. Der Vergleich zeigt deutlich die schlechtere Bildauflösung der Last-image-hold-Aufnahme.

\section{Abbildung 7}

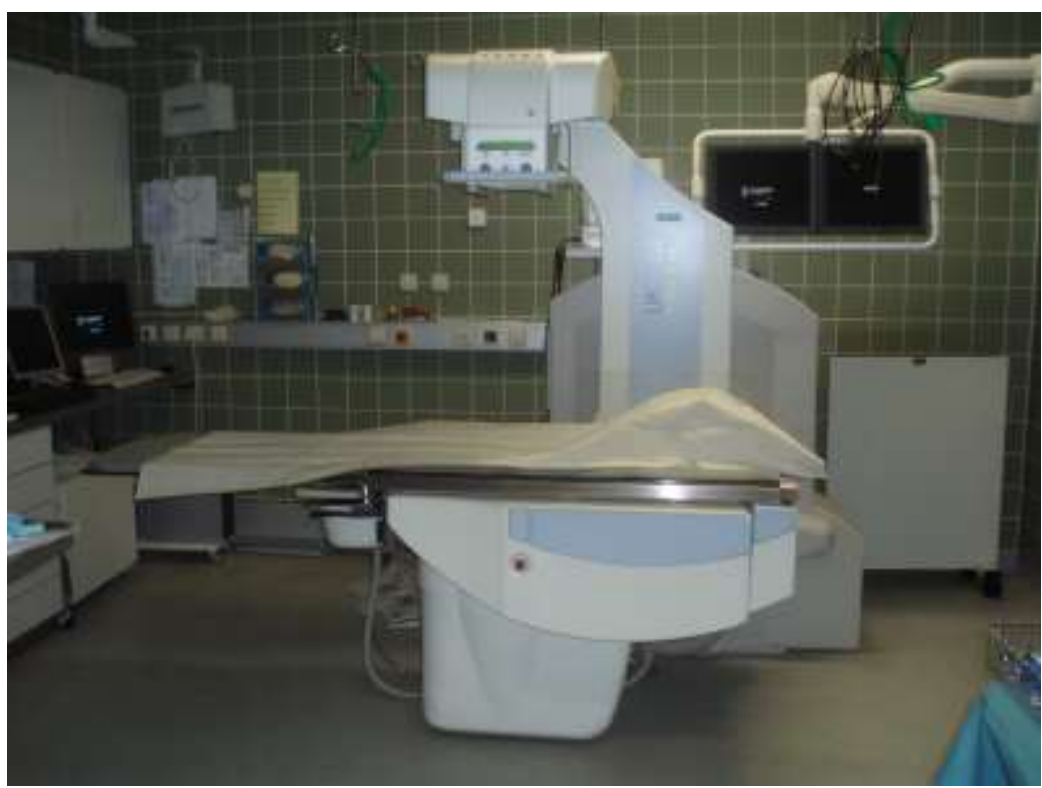

Röntgenarbeitsplatz Uroskop Access an der Klinik für Urologie und Kinderurologie des Klinikums Bad Hersfeld. 
In den Strahlengang fest eingebracht ist eine Dosismesskammer zur Messung des Dosis-Flächen-Produktes, gemessen in $\mu \mathrm{Gy}^{*} \mathrm{~m}^{2}$.

Die Durchleuchtungsbilder sowie die Röntgenaufnahmen werden auf TFT-Bildschirmen an einem Bewegungsarm des Uroskop Access sowie auf TFTBildschirmen an der Befundungs- und Nachverarbeitungsstation angezeigt.

Während der Durchleuchtung und der Anfertigung einer Röntgenaufnahme werden die notwendigen Parameter zur Berechnung der Strahlenbelastung wie Durchleuchtungszeit, Röhrenspannung, Anzahl der Röntgenbilder und Lastimage-hold-Aufnahmen sowie das resultierende Dosis-Flächen-Produkt (gemessen in $\left.\mu G y^{*} m^{2}\right)$ kontinuierlich registriert und am Ende der Operation zusammen mit den Röntgenbildern im Bildverarbeitungs- und -dokumentationssystem Flurospot Compact der Fa. Siemens, im krankenhausinternen DICOM-System (Medos 8.42) und zusätzlich auf dem Operationsprotokoll dokumentiert.

Die Bildbefundung und eine mögliche digitale Bildnachverarbeitung wie Helligkeits- und Kontrastveränderungen, Kantenanhebung oder Bildbeschriftungen erfolgen an der Arbeits- und Dokumentationstation mit dem Bildverarbeitungssystem FLUOROSPOT Compact der Fa. Siemens. 


\subsection{Ureterorenoskopische Steinentfernung}

\subsubsection{Prinzip der Ureterorenoskopie}

Die erste Ureterorenoskopie wurde bereits 1912 von Young beschrieben (Knoll und Alken 2006). Die Ureterorenoskopie wurde seither immer weiter verbessert und hat insbesondere in den letzten Jahren eine rasante gerätetechnische Weiterentwicklung erlebt. Durch die ständige Verkleinerung und Verbesserung der optischen Systeme sowie durch die Weiterentwicklung von flexiblen Endoskopen können heute alle Bereiche des Harnleiters und des Nierenbeckenkelchsystems retrograd endoskopisch erreicht werden. Dadurch gewann die Ureterorenoskopie in den letzten Jahren zunehmend an Bedeutung. Neben der diagnostischen Inspektion von Harnleiter, Nierenbecken und Nierenkelchen erlaubt die Ureterorenoskopie auch endoskopisch operative Eingriffe am oberen Harntrakt. Hierzu gehören die endoskopischen Probenentnahmen aus Harnleiter und Nierenbeckenkelchsystem (bei Verdacht auf ein Urothelkarzinom des oberen Harntraktes) und die endoskopische Inzision von Harnleiterengen. Zahlenmäßig weitaus am häufigsten wird die Ureterorenoskopie aber zur Steintherapie im oberen Harntrakt eingesetzt.

Bei der Ureterorenoskopie müssen semirigide oder flexible Endoskope retrograd in den Harnleiter eingeführt werden. Dieser Schritt wird durch eine ca. 3 Tage bis 2 Wochen zuvor eingelegte Ureterschiene (DJ-Schiene) deutlich vereinfacht, da die Harnleiterschiene zu einer Relaxierung der Harnleiterwand mit Weitstellung des Harnleiterlumens führt. In der Klinik für Urologie und Kinderurologie Bad Hersfeld erfolgt deshalb in Vorbereitung einer ureterorenoskopischen Steinentfernung im Vorfeld elektiv die Einlage einer Ureterschiene, sofern dies nicht bereits im Rahmen der Akuttherapie (z.B. bei therapierefraktären Koliken) notwendig gewesen ist.

Die Ureterorenoskopie selbst wird in der Regel in einer Allgemeinanästhesie mit Lagerung des Patienten in einer modifizierten Steinschnittlagerung durchgeführt. Bei der Ureterorenoskopie werden Endoskopien mit semirigiden Instrumenten (semirigide Ureterorenoskopie) und Endoskopien mit flexiblen Geräten (flexible Ureterorenoskopie) unterschieden. 


\section{Semirigide Ureterorenoskope}

Für die semirigide Ureterorenoskopie stehen an der Klinik für Urologie und Kinderurologie des Klinikum Bad Hersfeld Ureterorenoskope der Firma Storz, Tuttlingen, Deutschland zur Verfügung. Die Ureterorenoskope (Gerätebezeichnung $27002 \mathrm{~K}$ ) haben eine Länge von $43 \mathrm{~cm}$ und einen zunehmend konischen Durchmesser von 9,5 Charr. an der Endoskopspitze bis 13,5 Charr. am Schaftende. Sie verfügen über ein Stablinsensystem (Hopkins-Stablinsen-System), das in einem begrenzten Umfang eine Biegung des Geräts erlaubt (semirigides Ureterorenoskop). In die Endoskope integriert sind ein Spülkanal zur kontinuierlichen Spülung des Harntrakts während der Endoskopie und ein separater Arbeitskanal von 5 Charr. Durchmesser, der das Einführen von Arbeitsgeräten (PE-Zangen, Dormiakörbchen, LASER-Fasern) erlaubt (Abb.8).

\section{Flexible Ureterorenoskope}

Zur flexiblen Ureterorenoskopie verfügt die Klinik für Urologie und Kinderurologie des Klinikums Bad Hersfeld ebenfalls über Geräte der Fa. Karl Storz, Tuttlingen, Deutschland (Gerätebezeichnung Flex- $X^{2}$ ). Es handelt sich um Geräte mit Fiberglasoptiken, die eine aktive Biegung der Endoskope an der Gerätespitze in 2 Ebenen (positiv und kontrapositiv) von mehr als $270^{\circ}$ erlauben. Der Gerätedurchmesser beträgt 7,5 Charr., die Arbeitslänge $67 \mathrm{~cm}$. Integriert in die Geräte sind ein separater Spülkanal zur kontinuierlichen Spülung des Harntraktes sowie ein Arbeitskanal von 3,6 Charr., über den dünne Arbeitsgeräte wie Dormiakörbchen oder LASER-Fasern vorgeführt werden können (Abb.9).

Die Ureterorenoskopie erfolgt unter endoskopischer und radiologischer Kontrolle (siehe 2.2 Radiologischer Arbeitsplatz und Dosisdokumentation). Die radiologische Kontrolle dient der Orientierung und der Lagekontrolle der Ureterorenoskope im Harnleiter und Nierenbeckenkelchsystem. Gleichzeitig können pathologische Befunde wie röntgendichte Steine oder Tumoren (nach Kontrastmittelgabe) radiologisch dokumentiert werden. Diese radiologische Dokumentation der verschiedenen operativen Schritte bei einer Ureterorenoskopie dient auch der justiziablen Dokumentation des operativen Eingriffes. 


\section{Abbildung 8}

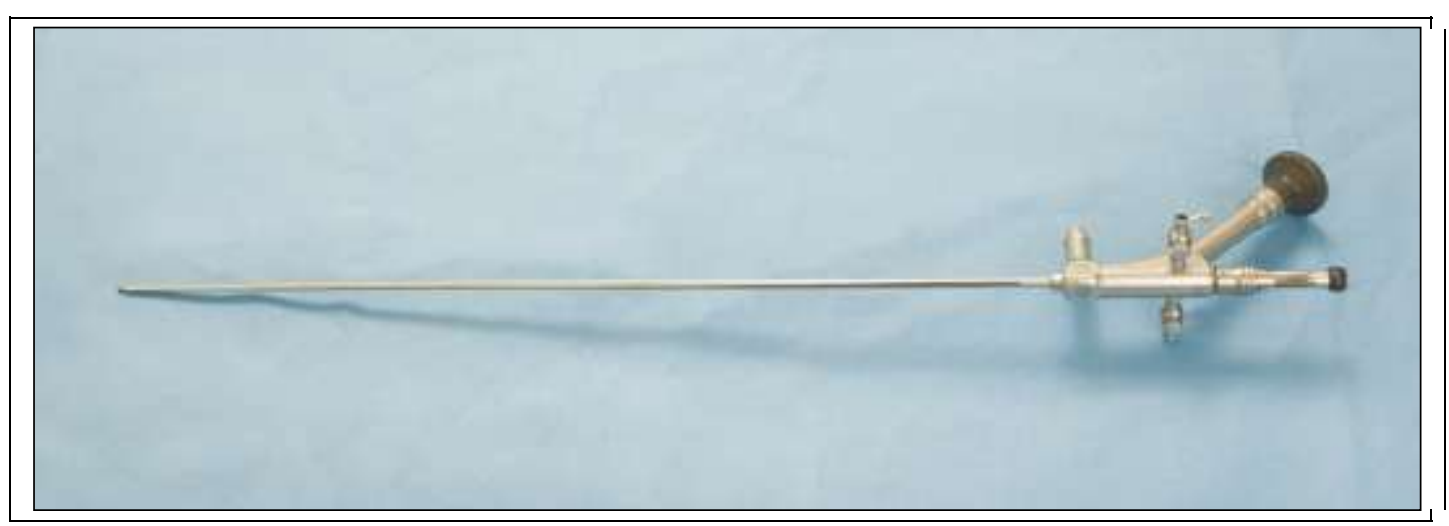

Semirigides Ureterorenoskope 27011 K, Fa. Storz, Tuttlingen, Deutschland.

Abbildung 9

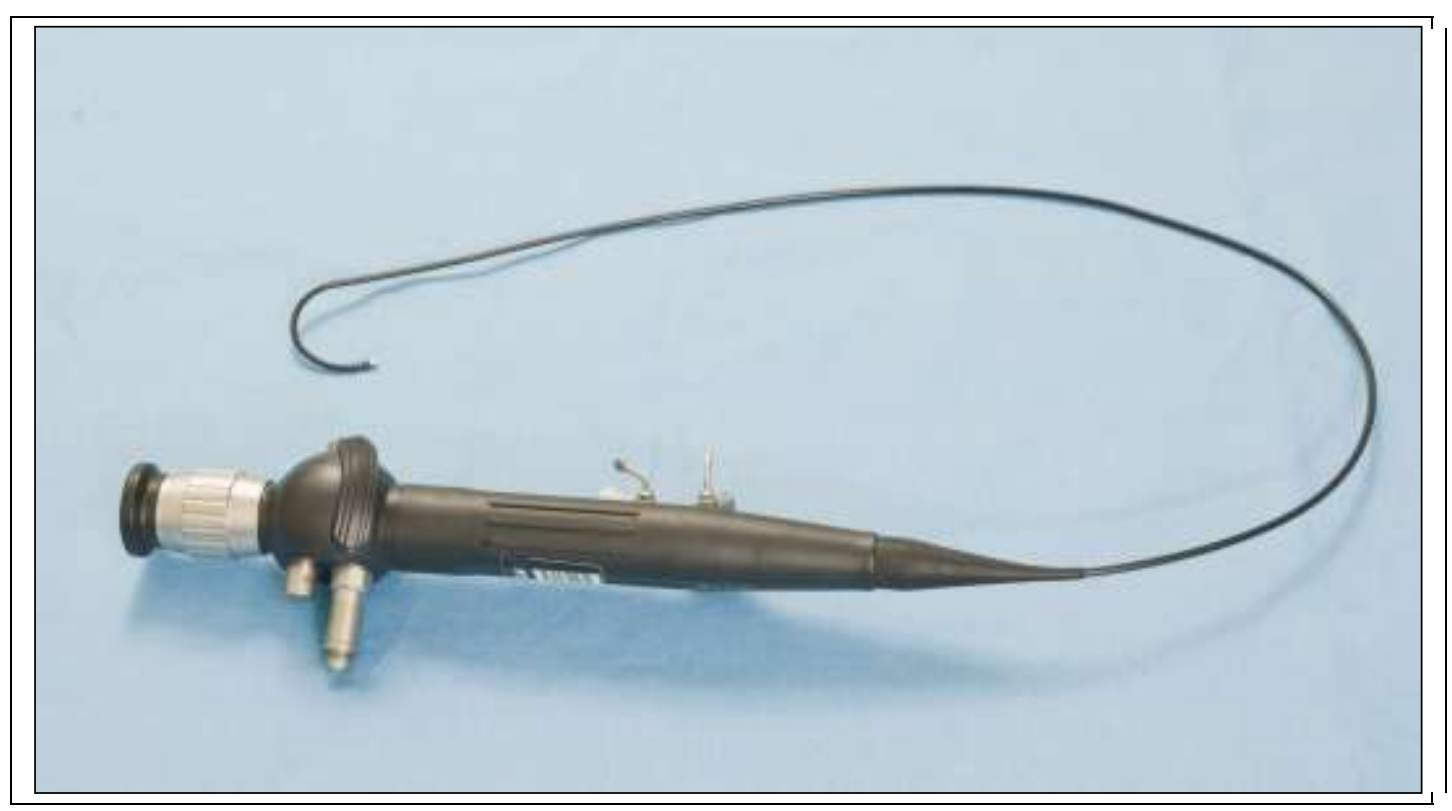

Flexibles Ureterorenoskop Flex- $X^{2}$ der Firma Karl Storz, Tuttlingen, Deutschland. 
Das endoskopische Bild wird heute in der Regel über Kamerasysteme auf einen Monitor (Videokette) übertragen, was die Durchführung des Eingriffes für den Operateur vereinfacht und dem assistierenden Personal eine gezielte Mitarbeit bei den einzelnen Operationsschritten ermöglicht. An der Klinik für Urologie und Kinderurologie des Klinikums Bad Hersfeld steht hierfür ein Videosystem der Firma Storz, bestehend aus der Xenon-Lichtquelle XENON 300, aus der Kameraeinheit SCB image 1, aus dem Bilddokumentationssystems AIDA und aus zwei TFT-Bildschirmen, zur Verfügung.

Neben der Übertragung des endoskopischen Bildes auf die TFT-Monitore des Videoturmes kann das Bild zudem auf den zweiten Monitor des digitalen Bildwandlersystems Siemens Uroskop Access eingeblendet werden, so dass endoskopisches Bild und radiologisches Bild für den Operateur in Blickrichtung nebeneinander stehen (Abb.10).

Die Ureterorenoskopie wird normalerweise mit semirigiden Endoskopen begonnen. Diese werden wie bei einer Zystoskopie über die Harnröhre in die Harnblase vorgeführt. Nach einer Übersichtsendoskopie der Harnblase wird dann die liegende Harnleiterschiene (Doppel-J-Katheter) mit einer Fasszange gefasst und entfernt. Danach kann das semirigide Ureterorenoskop meist problemlos über das Ureterostium in den Harnleiter hochgeführt werden. Bei Passageschwierigkeiten wird das Hochführen des Ureterorenoskopes durch Vorschieben eines hydrophilen Führungsdrahtes erleichtert. Um eine Endoskopie des Harnleiters zu ermöglichen, muss der Harnleiter während der Ureterorenoskopie durch einen kontinuierlichen Spülstrom (physiologische Kochsalzlösung) aufgeweitet werden. Der Spülkanal erlaubt auch die Kontrastmittelgabe (retrograde Pyelographie) zur radiologischen Markierung des Hohlsytems und zur radiologischen Höhendokumentation pathologischer Befunde (z.B. von Kontrastmittelaussparungen bei intraluminalem Harnleitertumor).

Es erfolgt dann unter simultaner endoskopischer Sicht und radiologischer Bildwandlerkontrolle der ureterorenoskopische Eingriff, z. B. die Biopsie von Harnleitertumoren oder die ureterorenoskopische Steinentfernung (siehe 2.3.2) (Abb.11). 


\section{Abbildung 10}

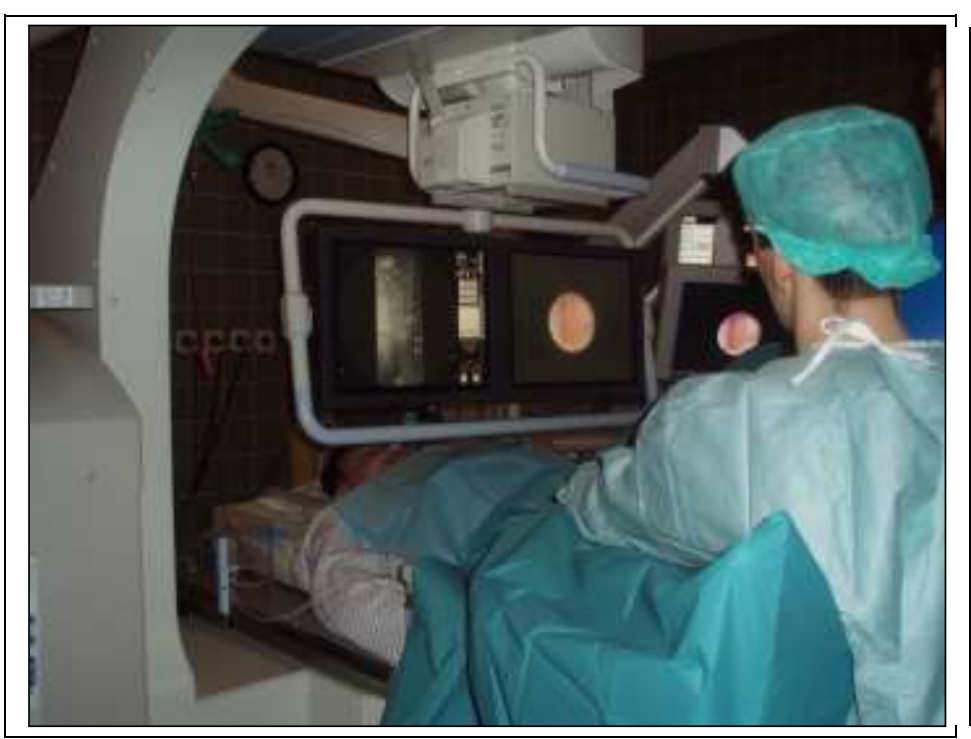

Parallele Darstellung von radiologischem Bild und endoskopischem Bild auf den beiden Monitoren des digitalen Röntgenarbeitsplatzes (Access Fa. Siemens).

\section{Abbildung 11}

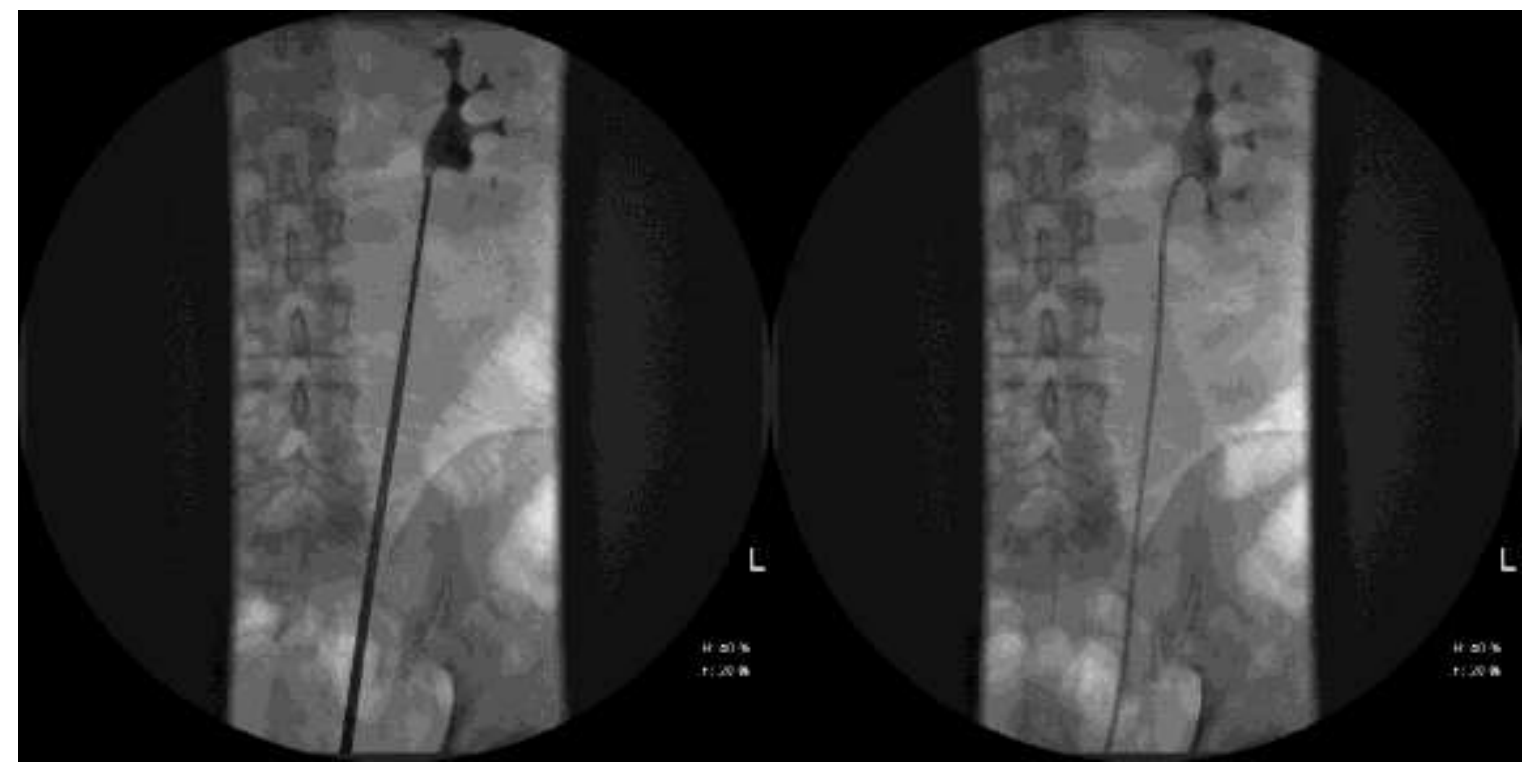

Radiologische Kontrolle bei semirigider und flexibler Ureterorenoskopie mit Lage des semirigiden Ureterorenoskopes unterhalb des Nierenbeckenabgangs (linkes Bild) und der Spitze des flexiblen Ureterorenoskopes in einer unteren Kelchgruppe (rechtes Bild). 
Kann mit den semirigiden Ureterorenoskopen keine ausreichende Darstellung des Harnleiters erreicht werden (z. B. wegen nicht passierbarer Harnleiterbiegungen) oder liegen die pathologischen Prozesse ungünstig in einer nicht erreichbaren mittleren oder unteren Kelchgruppe der Niere, so muss die semirigide Ureterorenoskopie durch eine flexible Ureterorenoskopie ergänzt werden.

Die Orientierung mit den dünnlumigen flexiblen Ureterorenoskopen in der Harnblase ist schwierig, und ein primäres Einführen des Ureterorenoskopes über das Ostium in den Harnleiter gelingt meist nicht. Deshalb erfolgt das Einführen des Geräts in den Harnleiter und das Hochführen des Gerätes zur Niere über hydrophile Führungsdrähte, die im Rahmen der semirigiden Ureterorenoskopie vorgelegt werden müssen. Zur besseren Orientierung im verzweigten Nierenbeckenkelchsystem wird das Nierenhohlsystem mit Kontrastmittel gefüllt, so dass die Lage des flexiblen Ureterorenoskopes im Kelchsystem jederzeit radiologisch kontrolliert werden kann. Auch die flexible Ureterorenoskopie erfolgt unter kontinuierlichem Spülstrom zur Aufweitung des Hohlsystems. Operative Eingriffe über das flexible Ureterorenoskop sind aufgrund der kleineren Arbeitskanäle und den daraus resultierenden kleineren Arbeitsgeräten technisch schwieriger als endoskopische Eingriffe über die großlumigeren semirigiden Ureterorenoskope.

Am Ende des operativen Eingriffes muss entschieden werden, ob zur Sicherung des Harnabflusses aus der Niere die erneute Einlage einer Ureterschiene notwendig ist (z. B. bei Schleimhautverletzungen oder Blutungen aus dem oberen Harntrakt), oder ob auf eine Harnleiterschiene verzichtet werden kann (z. B. nach problemloser Steinentfernung). 


\subsubsection{Prinzip der ureterorenoskopischen Steinentfernung}

Nachdem der Harnleiter- oder Nierenstein ureterorenoskopisch lokalisiert und dargestellt worden ist, muss der Harnstein aus dem Harntrakt entfernt werden. Prinzipiell lassen sich dabei kleinere Konkremente aus dem Harntrakt ohne weitere Desintegration in toto bergen. Hierfür werden entweder Steinfasszangen oder Steinfasskörbchen (Dormiakörbchen) verwendet (Abb.12).

Mit den Steinfasszangen werden die Harnsteine direkt gefasst und anschließend unter endoskopischer Sicht aus dem Harnleiter gezogen. Sind mehrere Konkremente zu erwarten, so können die Steine in der Harnblase zwischengelagert und am Ende der Operation ausgespült werden. Bei solitärem Stein wird der Stein sofort weiter durch die Harnblase und durch die Urethra nach außen gezogen. Nachteil der Steinfasszange ist, dass der Stein leicht aus den Branchen der Fasszange abrutscht; fasst man dagegen mit der Fasszange fester zu, so besteht die Gefahr, dass Teile des Steines abbrechen und Steinfragmente im Harnleiter zurückbleiben.

Aus diesem Grund erfolgt die Steinbergung an der Klinik für Urologie und Kinderurologie des Klinikum Bad Hersfeld in der Regel mittels sogenannter Dormiakörbchen. Es handelt sich dabei um Steinfasskörbchen, die im geschlossenen Zustand am Stein vorbeigeführt werden können. Bei Öffnen des Dormiakörbchens entfaltet sich ein Steinkörbchen aus spiralförmig oder längs angeordneten Metalldrähten. Das Körbchen wird um den Stein gelegt und anschließend zugezogen. Dabei umschließen die Metalldrähte den Harnstein fest, so dass er sicher aus dem Harntrakt gezogen werden kann (Abb.13).

Für die semirigide und flexible Ureterorenoskopie stehen in der Klinik für Urologie und Kinderurologie des Klinikums Bad Hersfeld Steinfasszangen der Fa. Storz in einer Größe von 3 - 5 Charr. und Dormiakörbchen in einer Größe von 2,2 - 2,5 Charr. zur Verfügung (Fa. Coloplast, Hamburg, Deutschland; Fa. Uromed, Oststeinbek, Deutschland). Als Besonderheit kommen im räumlich engen Nierenkelchsystem spitzenlose (tipless) Dormiakörbchen aus einer Nickel-TitanLegierung (Nitinol) der Fa. Uromed, Oststeinbek, Deutschland zum Einsatz. 


\section{Abbildung 12}

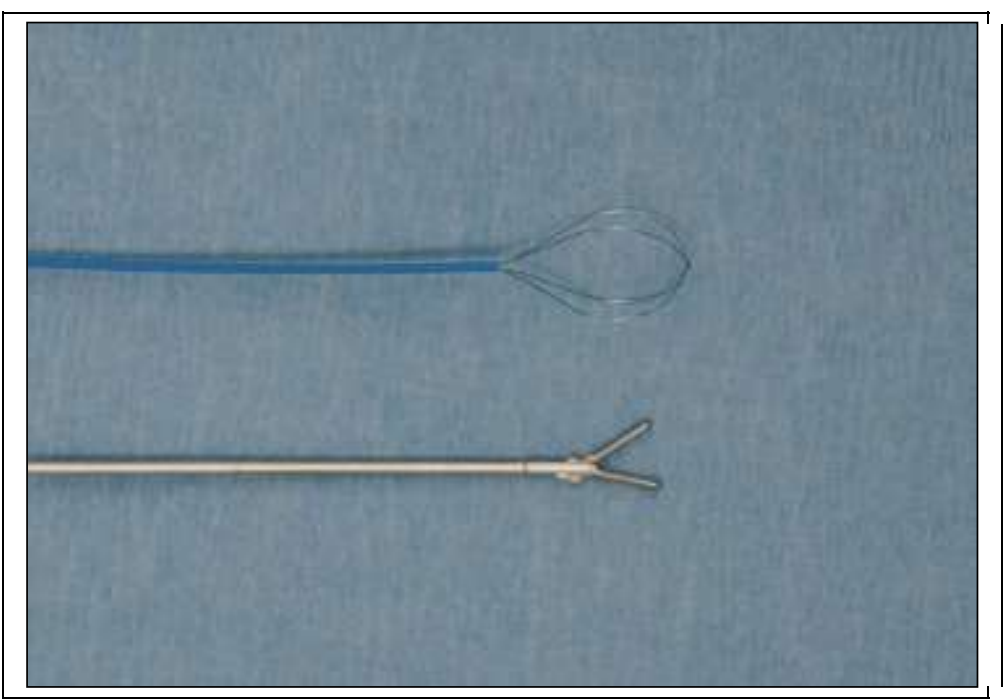

Dormiakörbchen (oben) und Steinfasszange zur Steinbergung.

\section{$\underline{\text { Abbildung } 13}$}

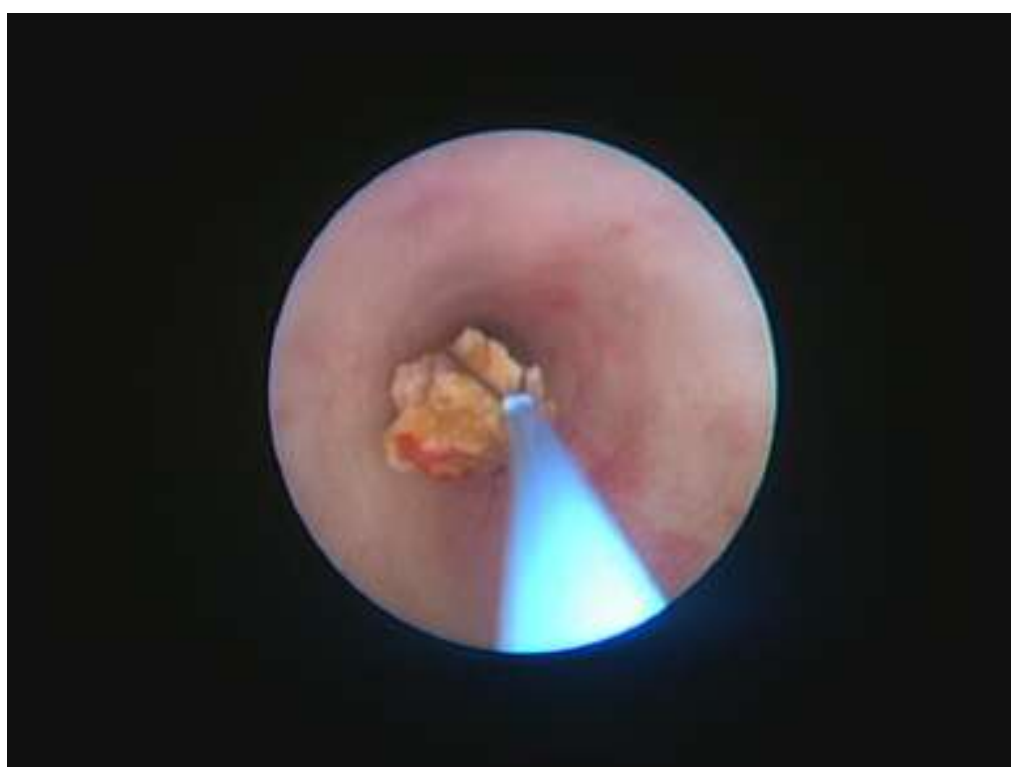

Endoskopischer Blick auf einen Harnleiterstein, der sicher in einem Dormiakörbchen gefasst ist. 
Größere Harnleiter- und Nierensteine, die nicht primär mit einem Dormiakörbchen gefasst werden können, die aufgrund ihrer Größe nicht durch den relativ engen Harnleiter entfernt werden können, oder die sich bei der Steinbergung an physiologischen Engstellen des oberen Harntraktes (Nierenbeckenabgang, Gefäßkreuzung des Harnleiters über die lliaca-communis-Gefäße, Ureterostium) verklemmen, müssen zunächst vor Ort im Körper desintegriert werden (intrakorporale Lithotripsie). Zur intrakorporalen Lithotripsie stehen unterschiedliche Gerätesystem zur Verfügung (www.urologenportal.de 2009).

\section{Ballistische Lithotripsie}

Bei der ballistischen Lithotripsie wird druckluftgesteuert im Gerätehandgriff ein Metallschlegel gegen ein Metallrohr geschlagen. Wie bei einem Presslufthammer entstehen dabei an der Spitze des Metallrohres Bewegungsauslenkungen in Längsrichtung, durch die der Stein mechanisch fragmentiert wird. Aufgrund des starren Metallrohrs ist die ballistische Lithotripsie nur in semirigiden Ureterorenoskopen im Harnleiter einsetzbar.

\section{Ultraschallvermittelte Lithotripsie}

Bei der ultraschallvermittelten Lithotripsie werden über ein piezoelektrisches Element hochfrequente Vibrationen (Ultraschall) erzeugt. Über ein starres Hohlrohr wird die Energie auf die Hohlrohrspitze übertragen. Bei direktem Steinkontakt führen die Vibrationen zu einer mechanischen Zerkleinerung des Harnsteines. Über das hohle Rohr kann dabei der entstehende Steinstaub kontinuierlich abgesaugt werden. Aufgrund des starren Metallrohrs ist auch die ultraschallvermittelte Lithotripsie nur bei der semirigiden Ureterorenoskopie im Harnleiter einsetzbar.

\section{Elektrohydraulische Lithotripsie}

Bei der elektrohydraulischen Lithotripsie werden durch Entladung einer Kondensatorenspannung ein Lichtbogen und nachfolgend eine Stoßwelle erzeugt. Diese Stoßwellen sprengen bei direktem Steinkontakt den Harnstein. Das System ist für die Therapie großer Blasensteine etabliert. 
Die Sonden für die elektrohydraulische Lithotripsie sind flexibel und können prinzipiell auch in den oberen Harntrakt vorgeführt werden. Die enorme Sprengkraft der Stoßwelle birgt aber das Risiko schwerwiegender Harnleiterwandverletzungen in sich, so dass diese Methode im oberen Harntrakt kaum angewendet wird.

\section{LASER-Lithotripsie}

Bei der LASER-Lithotripsie wird durch einen LASER-Impuls am Stein ein Kavitationsplasma erzeugt, das zur Zerstörung des Steinmaterials und zur Fragmentierung des Steins führt. Wesentlicher Vorteil der LASER-Lithotripsie ist, dass der LASER-Impuls über extrem dünne LASER-Fasern mit einem Durchmesser von ca. $200 \mu \mathrm{m}$ übertragen werden kann. Damit kann die LASER-Faser problemlos durch alle Ureterorenoskope inklusive der flexiblen Geräte vorgeschoben werden. Die LASER-Lithotripsie ist damit an jedem endoskopisch erreichbaren Ort im oberen Harntrakt möglich. Als LASER-Medien kommen Farbstoff-LASER, Neodym:YAG-LASER, Alexandrit-LASER und Holmium:YAGLASER zum Einsatz.

An der Klinik für Urologie und Kinderurologie des Klinikum Bad Hersfeld steht zur Lithotripsie ein Holmium:YAG-Laser $\left(\right.$ Sphin $x^{\circledR}$ ) der Firma LisaLaser, KatlenburgLindau, Deutschland, zur Verfügung (Abb.14). Das Sphin ${ }^{\circledR}$-LASER-System ist ein chirurgischer Laser, der neben der Steinlithotripsie auch im Kontakt- oder NichtKontakt-Betrieb für die chirurgische Koagulation von Weich- und Hartgewebe benutzt werden kann. Den Kern des Festkörper-LASER-Systems bilden 3-wertige Holmium-Ionen in einer Kristallmatrix aus synthetischem Yttrium-Aluminium (Holium:Yttrium-Aluminium-Garnet $=$ Holmium:YAG). Unter optischer Anregung durch das Licht einer Blitzlampe emittieren die Holmium-Ionen ein gepulstes LASER-Licht mit der Wellenlänge von 2,1 $\mu \mathrm{m}$. Der entstehende gepulste LASER Strahl wird über ein Glasfaserkabel aus Quarzglas aus dem LASER-System über das Ureterorenoskop zum Harnstein weitergeführt. Das Auftreffen des gepulsten LASER-Lichts auf den Stein und das daraus resultierende Kavitationsplasma führt in Abhängigkeit von der Steinzusammensetzung zur teilweisen oder völligen Fragmentierung des Steines. 
Abbildung 14

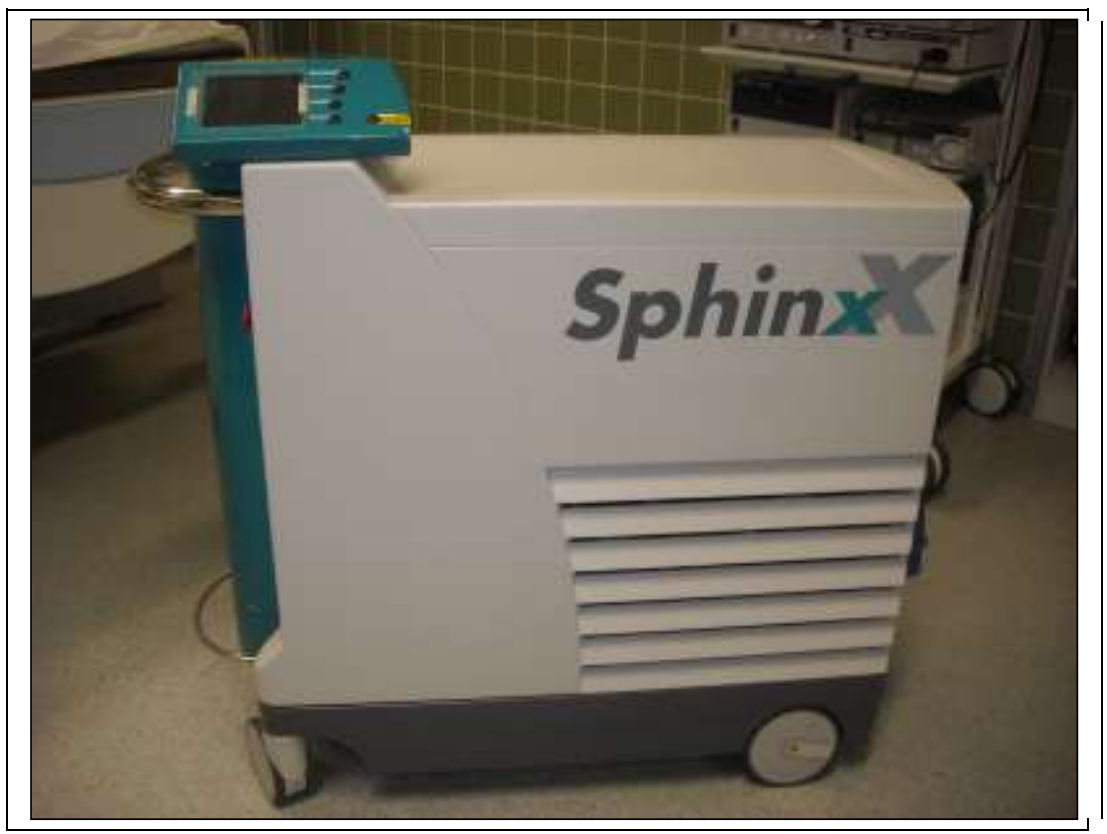

Sphinx ${ }^{\circledR}$ Holmium:YAG - Laser der Firma LisaLaser, Katlenburg-Lindau, Deutschland. 
Nach der erfolgreichen Steindesintegration müssen die entstandenen Steinfragmente mit den bereits oben dargestellten Methoden (Steinfasszange oder Dormiakörbchen) geborgen werden. Ziel ist die vollständige Steinfreiheit des betroffenen Harntraktes. Die Steinfreiheit wird mittels ureterorenoskopischer Ausspiegelung des gesamten oberen Harntraktes überprüft. Ist eine Steinfreiheit nicht sicher zu dokumentieren (z. B. aufgrund eingeschränkter endoskopischer Sicht bedingt durch Blutung im Nierenbeckenkelchsystem), so sollte eine Secondlook-Ureterorenoskopie im Abstand von wenigen Tagen erfolgen.

Bei der abschließenden Endoskopie des oberen Harntraktes wird auch überprüft, ob auf eine postoperative Ureterschiene verzichtet werden kann. Lassen Blutungen, Schleimhautödeme (Steinbett) oder Schleimhautverletzungen vermuten, dass der Abfluss aus dem betroffenen oberen Harntrakt postoperativ gestört sein könnte, so erfolgt zur Sicherstellung des Harnabflusses die erneute Einlage einer Ureterschiene, die dann nach ca. 7 Tagen entfernt werden kann.

Das entfernte Steinmaterial wird hinsichtlich der Steinzusammensetzung analysiert (in der Klinik für Urologie und Kinderurologie des Klinikum Bad Hersfeld mittels infrarotspektrometrischer Steinanalyse durch Labor Limbach, Heidelberg, Deutschland), um gegebenenfalls aus der Steinzusammensetzung Rückschlüsse für eine mögliche Steinmetaphylaxe gewinnen zu können. 


\subsection{Datenerhebung und statistische Methoden}

Zur Untersuchung wurden im Dokumentationssystem für operative Eingriffe an der Klinik für Urologie und Kinderurologie des Klinikum Bad Hersfeld alle Patienten ermittelt, bei denen zwischen dem 01.07.2008 und dem 01.07.2010 eine ureterorenoskopische Steinentfernung durchgeführt wurde. Aus den Krankenakten wurden dann Name, Geburtsdatum und Geschlecht, Diagnose, Datum des Eingriffs und der Operateur erfasst. Aus dem Operationsprotokoll waren Art der Operationsdurchführung (semirigide bzw. flexiblen Ureterorenoskopie, LASERLithotripsie, postoperative Harnleiterschienung) sowie die relevanten Daten zur Strahlenbelastung (Durchleuchtungszeit, Zahl der Röntgenaufnahmen, Lastimage-hold-Aufnahmen, Röhrenspannung und Dosis-Flächen-Produkt) ersichtlich.

Die Daten wurden in einer Datenbank unter Verwendung des Tabellenkalkulationsprogramms EXCEL 2003 for Windows (Fa. Microsoft, Redmond, USA) gespeichert. Die statistischen Angaben zu Mittelwert, Maximalwert und Minimalwert wurden im Tabellenkalkulationsprogramm ermittelt. Für die weiteren statistischen Auswertungen (one-way-Anova, t-Test- und Korrelations- bzw. Regressionsanalysen) wurden die Daten in das Statistikprogramm GraphPad Prims 5 (Firma GraphPad Inc., La Jolla, USA) überspielt und die statistischen Berechnungen mit diesem Programm durchgeführt. Das jeweils angewendete statistische Verfahren ist bei der Darstellung der Ergebnisse angegeben. $p$-Werte $<0,05$ wurden dabei als statistisch signifikant angesehen, $p$-Werte $<0,0001$ als statistisch hochsignifikant.

Die grafischen Darstellungen in Balkengrafiken wurden mit dem Tabellenkalkulationsprogramm EXCEL 2003 vorgenommen. Die Boxplot-Grafiken (Darstellung: minimaler Wert, maximaler Wert, Mittelwert, Boxplot 25 - $75 \%$ Perzentil) und die Punktwolkengrafiken (mit Darstellung von Korrelations- bzw. Regressionsgeraden) wurden mit Hilfe des Statistikprogramms GraphPad Prism5 erstellt. 


\section{Ergebnisse}

\subsection{Strahlenbelastung und Steinlokalisation}

Die Strahlenbelastung bei den 126 Patienten mit Harnleitersteinen, charakterisiert durch DFP, betrug minimal 10,7 $\mu \mathrm{Gy}^{*} \mathrm{~m}^{2}$, maximal 1983,4 $\mu \mathrm{Gy}^{*} \mathrm{~m}^{2}$, im Mittel 270,9 $\mu G y^{*} m^{2}$. Bei der ureterorenoskopischen Entfernung von Nierensteinen wurden bei 64 Patienten DFP-Werte von minimal 8,5 $\mu \mathrm{Gy}^{*} \mathrm{~m}^{2}$, maximal von $1733,0 \mu \mathrm{Gy}^{*} \mathrm{~m}^{2}$, im Mittel von 340,1 $\mu \mathrm{Gy}^{*} \mathrm{~m}^{2}$ angezeigt. Eine höhere Strahlenbelastung wurde angezeigt, wenn Steine aus Niere und Harnleiter geborgen werden mussten. Bei diesen 25 Patienten betrug der DFP-Wert minimal $57,5 \mu \mathrm{Gy}^{*} \mathrm{~m}^{2}$, maximal 3352,0 $\mu G y^{*} m^{2}$, im Mittel 744,2 $\mu \mathrm{Gy}^{*} \mathrm{~m}^{2}$ (Tab.5).

Beim Vergleich der Patientenkollektive fanden sich statistisch hochsignifikante Unterschiede in den einzelnen Behandlungsgruppen $(p<0,0001$, one-wayANOVA, hochsignifikant). Bei der Einzelanalyse zeigte sich dabei eine statistisch signifikant höhere Strahlenbelastung bei der Behandlung von Nierensteinen gegenüber der Behandlung von Harnleitersteinen ( $p=0,0304$, t-Test, signifikant). Eine hochsignifikant höhere Strahlenbelastung fand sich bei der simultanen Behandlung von Harnleiter- und Nierensteinen verglichen mit der Behandlung von Harnleitersteinen ( $p<0,0001$, t-Test, hochsignifikant), bzw. eine signifikant höhere Strahlenbelastung bei der simultanen Behandlung von Harnleiter- und Nierensteinen verglichen mit der alleinigen Behandlung von Nierensteinen $(p<0,0068$, tTest, hochsignifikant) (Abb.15). 


\section{Tabelle 5}

DFP $\left(\mu G y^{*} m^{2}\right)$ in Abhängigkeit von der Steinlokalisation.

\begin{tabular}{|l|c|c|c|}
\hline Steinlokalisation & Minimum & Maximum & Mittelwert \\
\hline Harnleiter & 10,7 & 1983,4 & 270,9 \\
\hline Niere & 8,5 & 1733,0 & 340,1 \\
\hline Harnleiter und Niere & 57,5 & 3352,0 & 744,2 \\
\hline
\end{tabular}

Abbildung 15

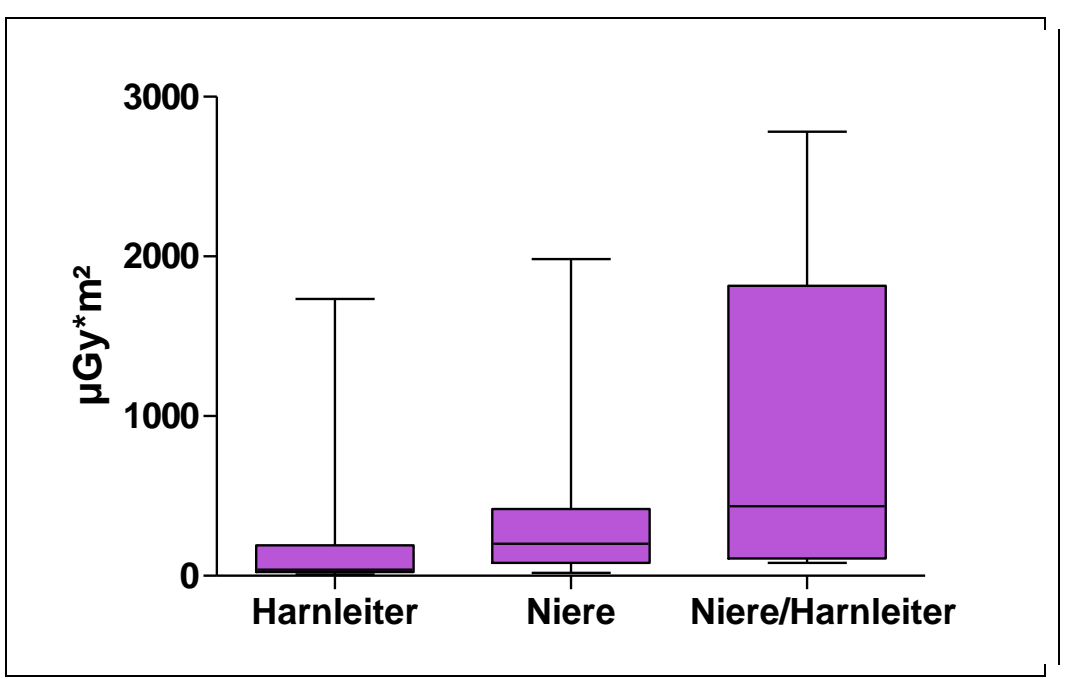

Strahlenbelastung $\left(\mu \mathrm{Gy}^{*} \mathrm{~m}^{2}\right)$ in Abhängigkeit von der primären Steinlokalisation im Harnleiter, in der Niere bzw. simultan sowohl in Niere wie auch im Harnleiter (Niere/Harnleiter). 


\subsection{Strahlenbelastung und Form der Ureterorenoskopie}

Wie bereits dargestellt beginnt die Ureterorenoskopie in der Regel mit semirigiden Ureterorenoskopen. Diese Ureterorenoskopie mit den semirigiden Endoskopen wurde dementsprechend bei allen 215 Patienten durchgeführt. Bei 159 Patienten musste aufgrund technischer Probleme, aufgrund der ungünstigen Steinlage in Nierenkelchen oder auch zur sicheren Dokumentation der Steinfreiheit eine Ureterorenoskopie mit einem flexiblen Ureterorenoskop angeschlossen werden.

Wurde eine alleinige semirigide Ureterorenoskopie durchgeführt (159 Patienten), so betrug das mittlere DFP $221,9 \mu \mathrm{Gy}^{*} \mathrm{~m}^{2}$ (minimal 8,5 $\mu \mathrm{Gy}^{*} \mathrm{~m}^{2}$, maximal 1304,1 $\mu G y^{*} m^{2}$ ). Musste zusätzlich ein flexibles Ureterorenoskop eingesetzt werden (56 Patienten), so lag das mittlere DFP mit 390,4 $\mu \mathrm{Gy}^{*} \mathrm{~m}^{2}$ (minimal $10,7 \mu \mathrm{Gy}^{*} \mathrm{~m}^{2}$, maximal 3352,0 $\mu \mathrm{Gy}^{*} \mathrm{~m}^{2}$ ) erwartungsgemäß deutlich höher. Dieser Unterschied war statistisch signifikant ( $p=0,0217$, t-Test, signifikant) (Abb.16).

Abbildung 16

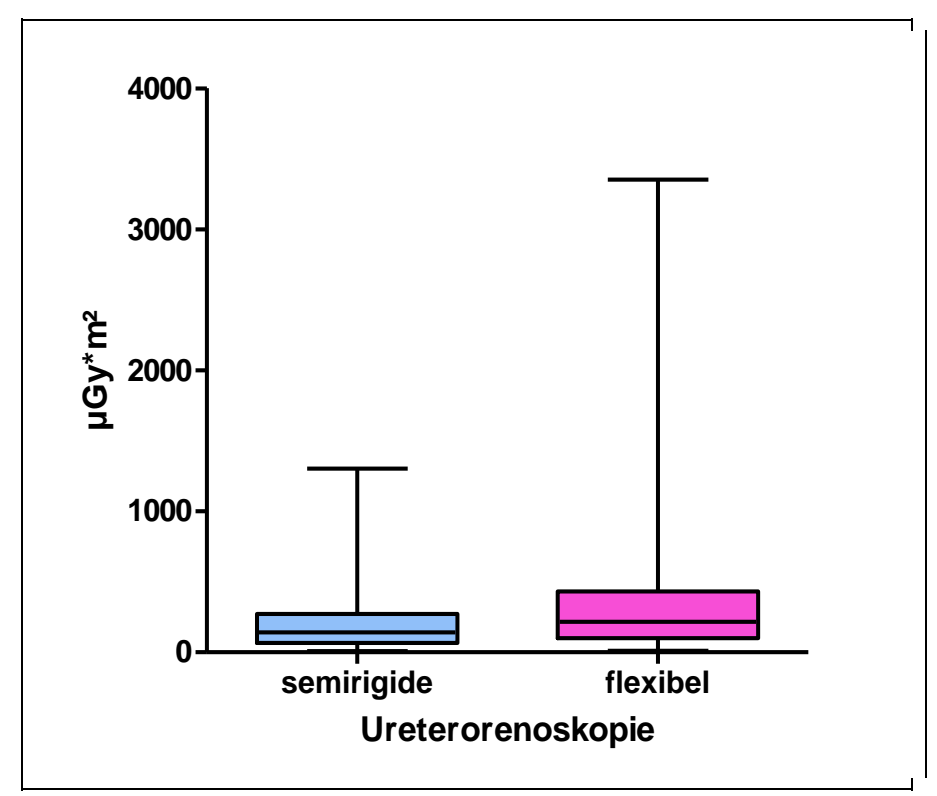

DFP in Abhängigkeit von der Form der Ureterorenoskopie (semirigide vs. flexibel). Die Unterschiede sind statistisch signifikant ( $p=0,0217$, $t$-Test). 


\subsection{Strahlenbelastung und Steingröße}

Die Steingröße im Röntgenbild (maximaler Steindurchmesser) variierte bei den Harnleitersteinen zwischen minimal 0,5 mm und maximal 16,4 $\mathrm{mm}$ (im Mittel 6,3 $\mathrm{mm}$ ) und bei den Nierensteinen zwischen minimal 0,5 mm und maximal 11,7 mm (im Mittel ebenfalls 6,3 mm). In der Regressionsanalyse ergab sich dabei keine enge Korrelation von Steingröße und Strahlenbelastung (Korrelationskoeffizient nach Pearson $r=0,233$; lineare Regression $r^{2}=0,054$ ) (Abb.17).

\section{Abbildung 17}

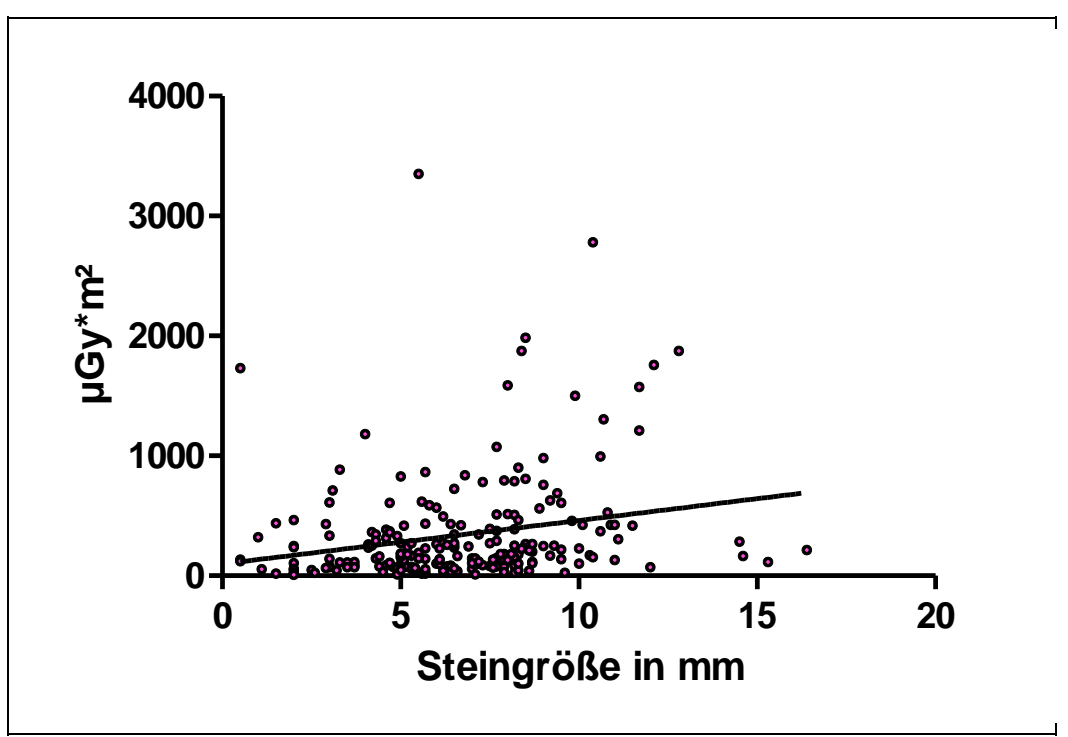

Lediglich schwache Korrelation zwischen der Steingröße und DFP (Pearsons Korrelationskoeffizient $r=0,233$; lineare Regression $r^{2}=0,054$ ). 


\subsection{Strahlenbelastung und LASER-Lithotripsie}

Bei 38 Patienten musste zur ureterorenoskopischen Steinentfernung eine LASERLithotripsie durchgeführt werden, bei 16 Patienten mit Harnleitersteinen, bei 13 Patienten mit Nierensteinen und bei 9 Patienten, die sowohl Harnleiter- als auch Nierensteine hatten. Die Steingröße lag zwischen minimal $1,1 \mathrm{~mm}$ und maximal 15,3 mm, im Mittel bei 8,4 mm.

Ohne den Einsatz der LASER-Lithotripsie betrug das durchschnittliche DFP 297,4 $\mu G y^{*} m^{2}$ (minimal 8,5 $\mu G y^{*} m^{2}$, maximal 1983,4 $\mu G y^{*} m^{2}$ ). Musste der Sphinx ${ }^{\circledR}$ Holmium:YAG-LASER zur Steindesintegration eingesetzt werden, stieg das durchschnittliche DFP auf 575,5 $\mu \mathrm{Gy}^{*} \mathrm{~m}^{2}$ (minimal 26,3 $\mu \mathrm{Gy}^{*} \mathrm{~m}^{2}$, maximal 3352,0 $\left.\mu G y^{*} m^{2}\right)$. Diese Unterschiede waren statistisch signifikant $(p=0,0007$, t-Test, signifikant) (Abb.18).

Abbildung 18

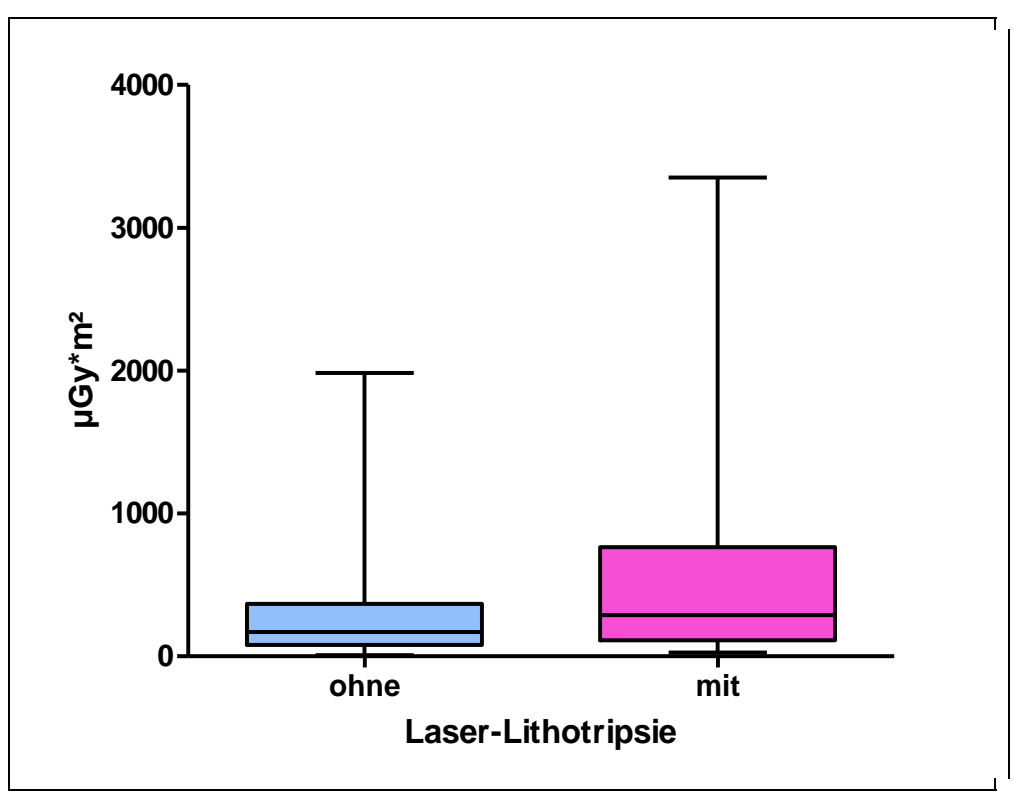

Signifikant erhöhtes DFP und damit erhöhte Strahlenbelastung der Patienten bei Notwendigkeit einer LASER - Lithotripsie ( $p=0,0007$, $t$-Test). 


\subsection{Strahlenbelastung und Durchleuchtungszeit}

Während der Ureterorenoskopie erfolgen einzelne technisch schwierige Operationsschritte oder radiologische Lagekontrollen des Ureterorenoskopes unter simultaner Röntgendurchleuchtung. Die notwendigen Durchleuchtungszeiten sind dabei unterschiedlich lang. Insgesamt ergaben sich Durchleuchtungszeiten zwischen 1 und 424 Sekunden, im Mittel betrug die Durchleuchtungszeit 61,0 Sekunden. Abbildung 19 zeigt eine enge Korrelation zwischen Durchleuchtungszeit und DFP (Korrelationskoeffizient nach Pearson $r=0,681$; lineare Regression $\left.r^{2}=0,464\right)$.

\section{$\underline{\text { Abbildung } 19}$}

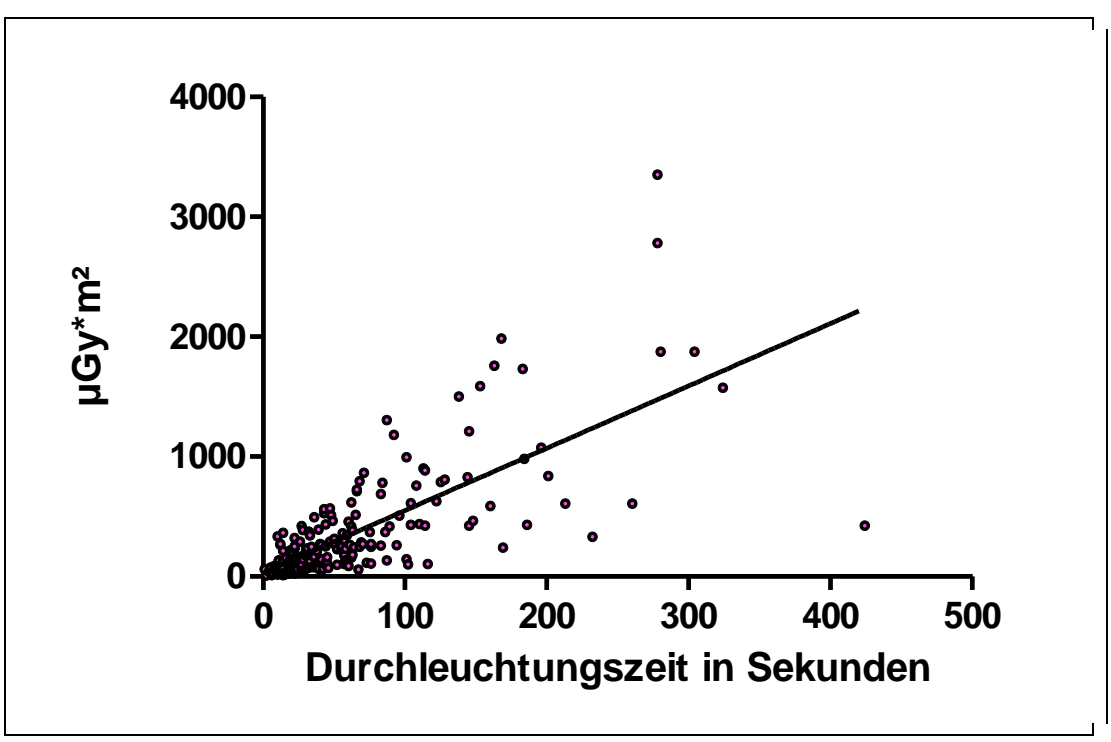

Enge Korrelation zwischen Durchleuchtungszeit und DFP (Korrelationskoeffizient nach Pearson $r=0,681$; lineare Regression $r^{2}=0,464$ ). 


\subsection{Strahlenbelastung und Anzahl der Röntgenaufnahmen}

Wichtige Operationsschritte wie die Dokumentation der Steinfreiheit oder die Neueinlage einer Harnleiterschiene am Operationsende sowie kritische Situationen wie eine Kontrastmittelextravasation werden als Röntgenbild, gegebenenfalls als Last-image-hold-Aufnahme des Durchleuchtungsbildes dokumentiert. Vor- und Nachteile der Last-image-hold-Aufnahmen wurde bereits ausführlich diskutiert (siehe 2.2 Methode). Bei einem von 215 Patienten erfolgten während der Ureterorenoskopie nur Last-image-hold-Aufnahmen, bei 167 Patienten ausschließlich Röntgenaufnahmen. Bei 47 Patienten wurden intraoperative Schritte sowohl mittels Röntgenbild als auch mittles Last-image-holdAufnahmen dokumentiert. Im Mittel erfolgten bei diesen 47 Patienten 4,9 Röntgenaufnahmen (minimal 1, maximal 16 Röntgenaufnahmen) und 3,8 Lastimage-hold-Aufnahmen (minimal 1, maximal 12 Last-image-hold-Aufnahmen). Die genaue Verteilung bei den 47 Patienten ist in Abb. 20 dargestellt.

Insgesamt erfolgten bei den 215 Patienten zwischen 0 und 16 Röntgenaufnahmen, im Mittel 4,3 Röntgenaufnahmen pro Untersuchung. In der Mehrzahl der Fälle (142 von 215 Patienten $=66,0 \%$ der Patienten) wurden intraoperativ zwischen 2 und 5 Röntgenaufnahmen erstellt. Die genaue Häufigkeitsverteilung der Anzahl der Röntgenaufnahmen ist in Abbildung 21 dargestellt.

Wie bereits dargelegt führen Last-image-hold-Aufnahmen im Gegensatz zur Dokumentation mittels Röntgenbild nicht zu einer zusätzlichen Strahlenbelastung. Allerdings fand sich in unserer Untersuchung nur eine mäßige Korrelation von zusätzlicher Dokumentation mittels Röntgenbild und Strahlenbelastung (Abb. 22) (Korrelationskoeffizient nach Pearson $r=0,5359$; lineare Regression $r^{2}=0,2872$ ). 


\section{Abbildung 20}

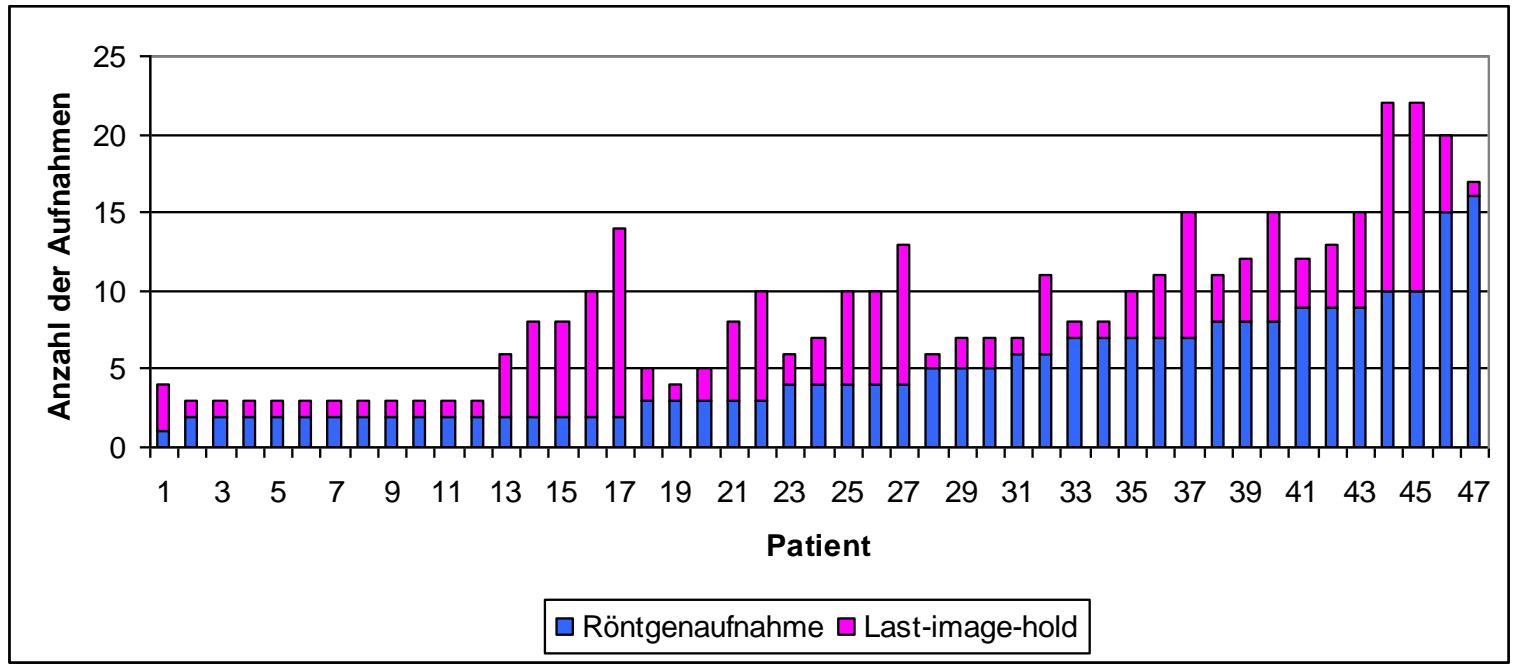

Verteilung der Anzahl von Röntgenaufnahmen und Last-image-hold-Aufnahmen bei den 47 Patienten, bei denen neben Röntgenaufnahmen auch Last-image-holdAufnahmen abgespeichert wurden.

Abbildung 21

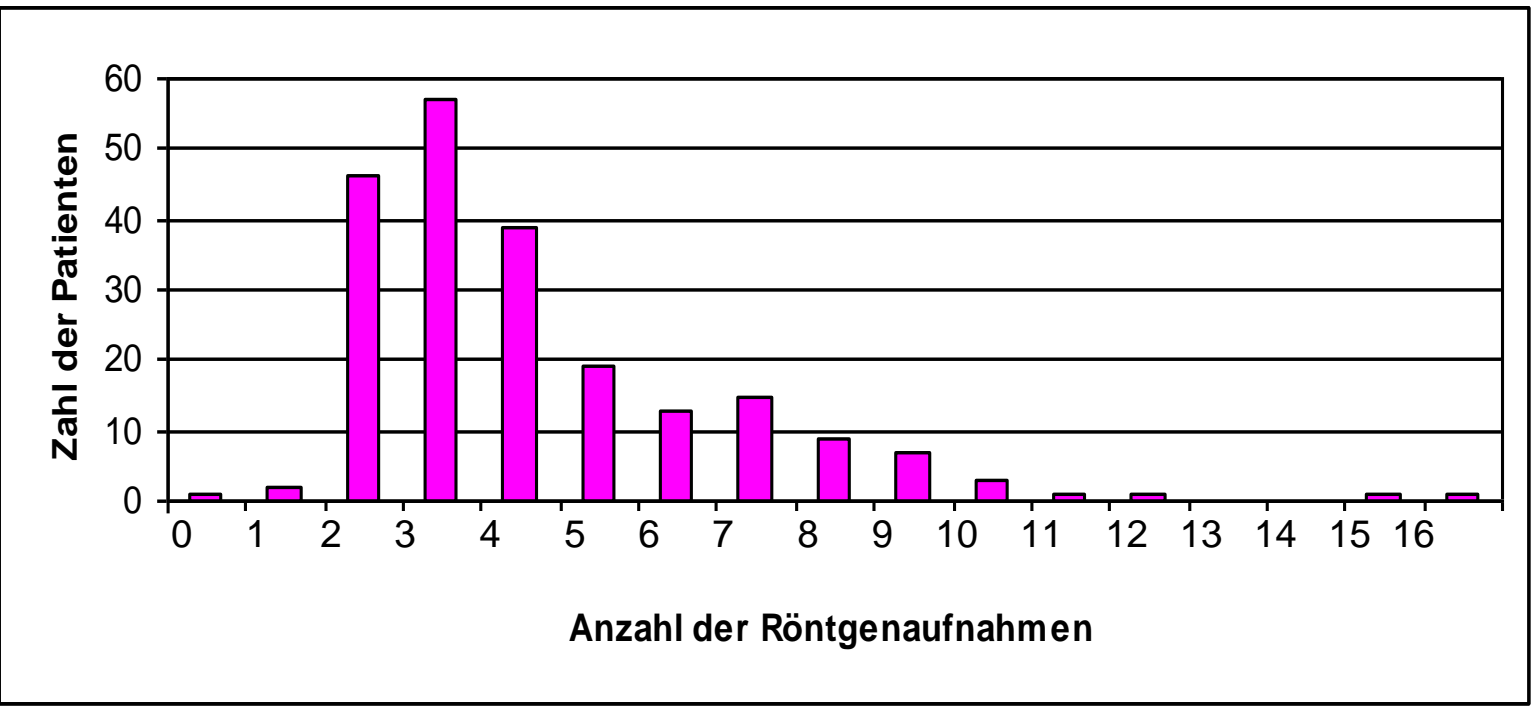

Verteilung der Anzahl an intraoperativen Röntgenaufnahmen pro Patient. 
Abbildung 22

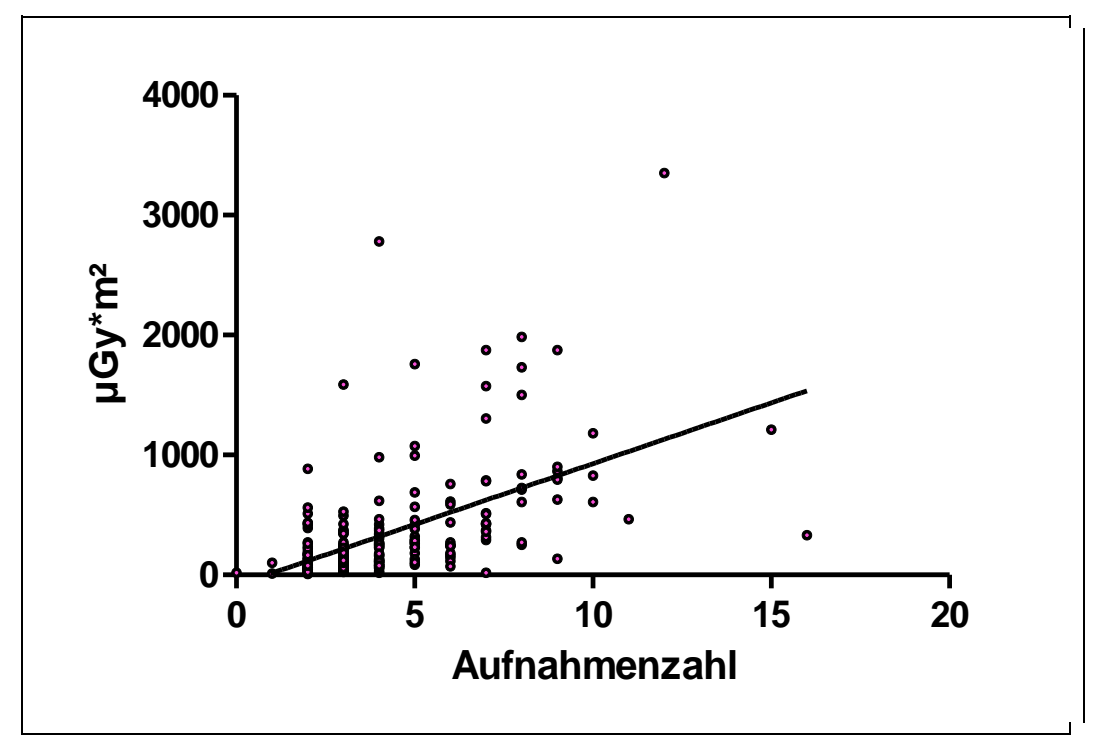

Mäßige Korrelation von intraoperativen Röntgenaufnahmen und DFP (Korrelationskoeffizient nach Pearson $r=0,5359$; lineare Regression $r^{2}=0,2872$ ). 


\subsection{Strahlenbelastung und Einlage einer postoperativen Harnleiterschiene}

Am Ende der ureterorenoskopischen Steinentfernung wurde der obere Harntrakt endoskopisch überprüft. Ließen Blutungen, Schleimhautödeme (Steinbett) oder Schleimhautverletzungen vermuten, dass postoperativ der Harnabfluss aus dem betroffenen oberen Harntrakt gestört sein könnte, so erfolgt zur Sicherstellung des Harnabflusses die Einlage einer postoperativen Ureterschiene. Die Entscheidung über die Neueinlage einer Harnleiterschiene oblag dem verantwortlichen Operateur. Eine postoperative Ureterschiene wurde bei 30 von 215 Patienten (= $13,9 \%$ der Patienten) eingelegt.

Ohne postoperative Harnleiterschienung betrug das DFP im Mittel 267,9 $\mu \mathrm{Gy}^{*} \mathrm{~m}^{2}$ (minimal 8,5 $\mu \mathrm{Gy}^{*} \mathrm{~m}^{2}$, maximal $1983,4 \mu \mathrm{Gy}^{*} \mathrm{~m}^{2}$ ). Musste dagegen eine postoperative Harnleiterschiene gelegt werden, so betrug das mittlere DFP 831,4 $\mu G y^{*} m^{2}$ (minimal 72,2 $\mu \mathrm{Gy}^{*} \mathrm{~m}^{2}$, maximal 3352,0 $\mu \mathrm{Gy}^{*} \mathrm{~m}^{2}$ ). Die Unterschiede waren statistisch hochsignifikant ( $p<0,0001$, t-Test, hochsignifikant) (Abb.23).

Abbildung 23

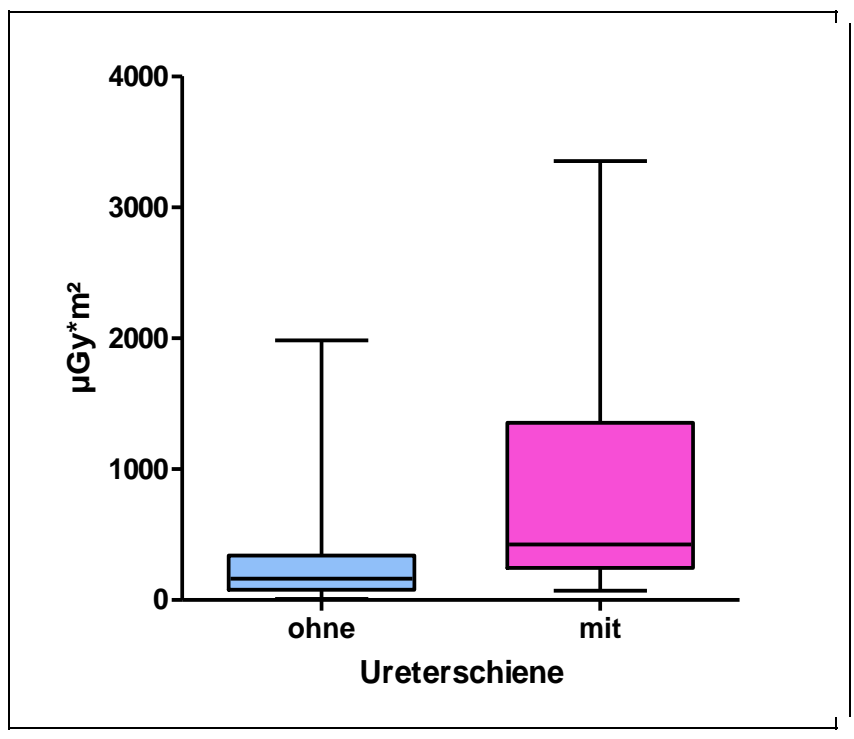

Hochsignifikant erhöhtes DFP und damit erhöhte Strahlenbelastung der Patienten bei Notwendigkeit zur Einlage einer postoperativen Ureterschiene $(p<0,0001$, tTest). 


\subsection{Strahlenbelastung und Operateure}

Insgesamt wurden die Patienten von drei erfahrenen Operateuren (ChA, OA1, OA2) und von zwei in der Ausbildung befindlichen Operateuren (WBA1, WBA2) unter fachärztlicher Aufsicht durchgeführt. Die Zahl der jeweils durchgeführten Operationen, die durchschnittliche Strahlenbelastung sowie minimal und maximal gemessene Strahlenbelastung pro Operation sind in Tabelle 6 detailliert dargestellt. Es zeigten sich dabei in der Gesamtbetrachtung statistisch signifikante Unterschiede ( $p=0,0010$, one-way-Anova, signifikant). Die insgesamt günstigste Strahlenbelastung wurde bei Operationen durch den Operateur OA1 gemessen. Diese Unterschiede waren gegenüber den Operateuren ChA und OA2 hochsignifikant (jeweils $p<0,0001$, t-Test, hochsignifikant), gegenüber dem sich in Facharztweiterbildung befindlichen Operateur WBA2 signifikant $(p=0,0425, t-$ Test, signifikant). Die weiteren Einzelanalysen mit dem Vergleich der Operateure untereinander ergaben keine statistisch signifikanten Unterschiede (Abb.24).

Für die drei Operateure mit Facharztstatus (ChA, OA1, OA2) wurde die intraoperative Strahlenbelastung weiter analysiert. Dabei zeigte sich, dass die Strahlenbelastung signifikant mit der intraoperativen Durchleuchtungszeit korrelierte (Tab.7 und Abb.25), dass aber auch hinsichtlich der intraoperativen Röntgenaufnahmen signifikante Unterschiede bestanden (Tab.8 und Abb.26). Interessant ist dabei die Analyse der Last-image-hold-Aufnahmen. Hier findet sich eine statistisch signifikant höhere Anzahl von Last-image-hold-Aufnahmen (ohne zusätzliche Strahlenbelastung) bei Operationen durch den Operateur ChA (Tab.9 und Abb.27).

Für die Analyse von intraoperativer Durchleuchtungszeit, Zahl der Röntgenaufnahmen und Zahl der Last-image-hold-Aufnahmen wurden die Operationen durch Weiterbildungsassistenten nicht berücksichtigt, da hier die Durchleuchtungszeit, das Durchführung von Röntgenaufnahmen und das Speichern von Lastimage-hold-Aufnahmen wesentlich durch den assistierenden und OP-verantwortlichen Facharzt beeinflusst wurde. 
Tabelle 6

Strahlenbelastung $\left(\mu G y^{*} m^{2}\right)$ bei ureterorenoskopischer Steinentfernung, aufgeschlüsselt nach Operateur.

\begin{tabular}{|l|l|c|c|c|c|}
\hline Name & Arztstatus & OP-Zahl & Mittelwert & Minimum & Maximum \\
\hline ChA & Chefarzt & 53 & 432,4 & 19,7 & 1983,4 \\
\hline OA1 & Oberarzt & 78 & 194,1 & 10,7 & 980,2 \\
\hline OA2 & Oberarzt & 43 & 538,1 & 20,3 & 3352,0 \\
\hline WBA1 & Weiterbildungsassistent & 17 & 295,0 & 8,5 & 1876,0 \\
\hline WBA2 & Weiterbildungsassistent & 24 & 345,8 & 26,1 & 2781,0 \\
\hline
\end{tabular}

\section{Abbildung 24}

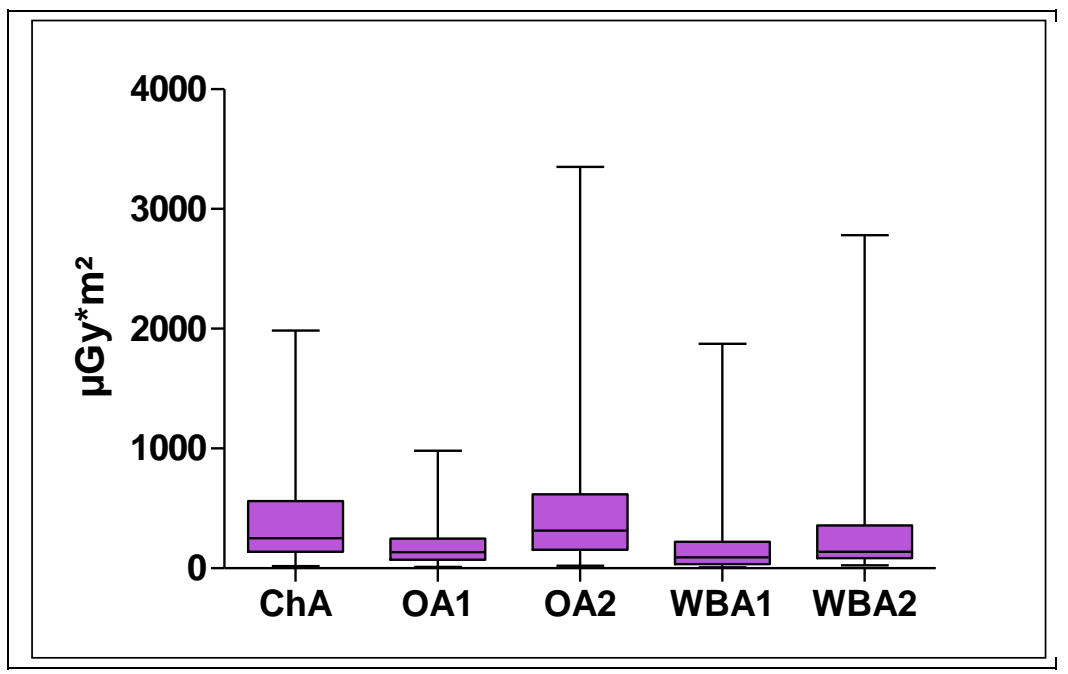

Strahlenbelastung bei der ureterorenoskopischen Steinentfernung, aufgeschlüsselt nach Operateur. Die statistische Analyse zeigte insbesondere eine günstig niedrige Strahlenbelastung bei Operationen durch den Operateur OA1 (statistisch hochsigifikant gegenüber ChA und OA2 ( $p<0,0001$, t-Test) und statistisch signifikant gegenüber WBA2 $(p=0,0425$, $t$-Test). 


\section{Tabelle 7}

Durchleuchtungszeit (in Sekunden) aufgeschlüsselt nach Operateuren mit Facharztstatus

\begin{tabular}{|l|l|c|c|c|}
\hline Name & Arztstatus & Mittelwert & Minimum & Maximum \\
\hline ChA & Chefarzt & 79,4 & 10 & 424 \\
\hline OA1 & Oberarzt & 43,2 & 5 & 232 \\
\hline OA2 & Oberarzt & 73,5 & 1 & 280 \\
\hline
\end{tabular}

Abbildung 25

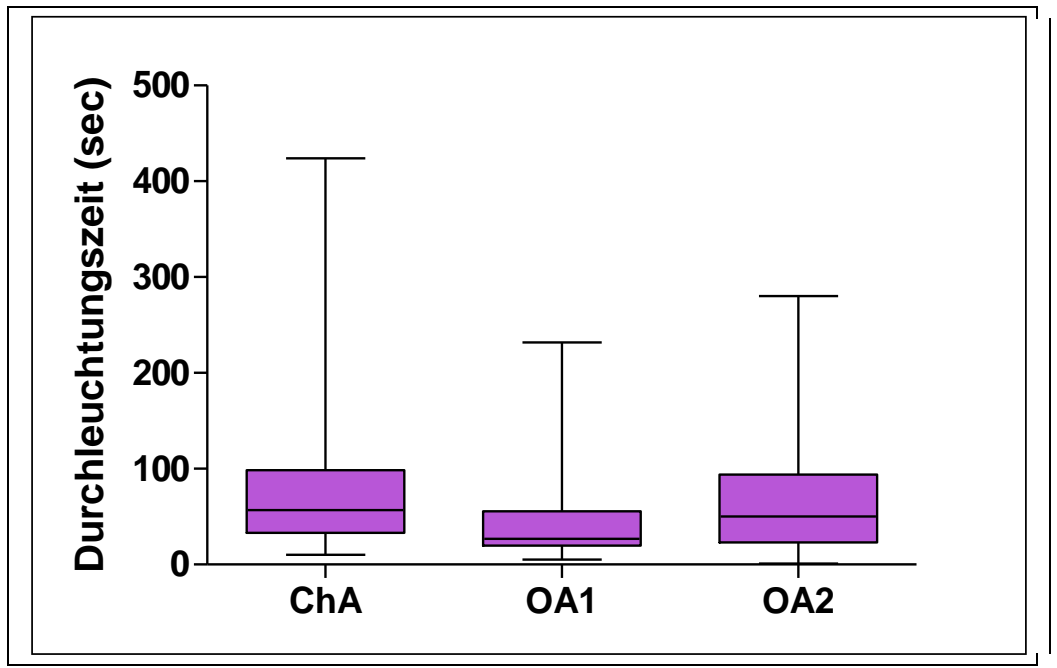

Durchleuchtungszeit bei der ureterorenoskopischen Steinentfernung, aufgeschlüsselt nach Operateuren mit Facharztstatus. Die statistische Analyse zeigte insbesondere eine günstig niedrige Durchleuchtungszeit bei Operationen durch den Operateur OA1 (statistisch signifikant gegenüber ChA und OA2 $(p=0,0004$ bzw. $p=0,0029$, $t$-Test). Der Vergleich von ChA und OA2 ergibt keine statistisch signifikanten Unterschiede ( $p=0,6919$, $t$-Test). 


\section{Tabelle 8}

Zahl der intraoperativen Röntgenaufnahmen aufgeschlüsselt nach Operateuren mit Facharztstatus

\begin{tabular}{|l|l|c|c|c|}
\hline Name & Arztstatus & Mittelwert & Minimum & Maximum \\
\hline ChA & Chefarzt & 5,2 & 0 & 15 \\
\hline OA1 & Oberarzt & 3,7 & 1 & 16 \\
\hline OA2 & Oberarzt & 5,0 & 2 & 12 \\
\hline
\end{tabular}

Abbildung 26

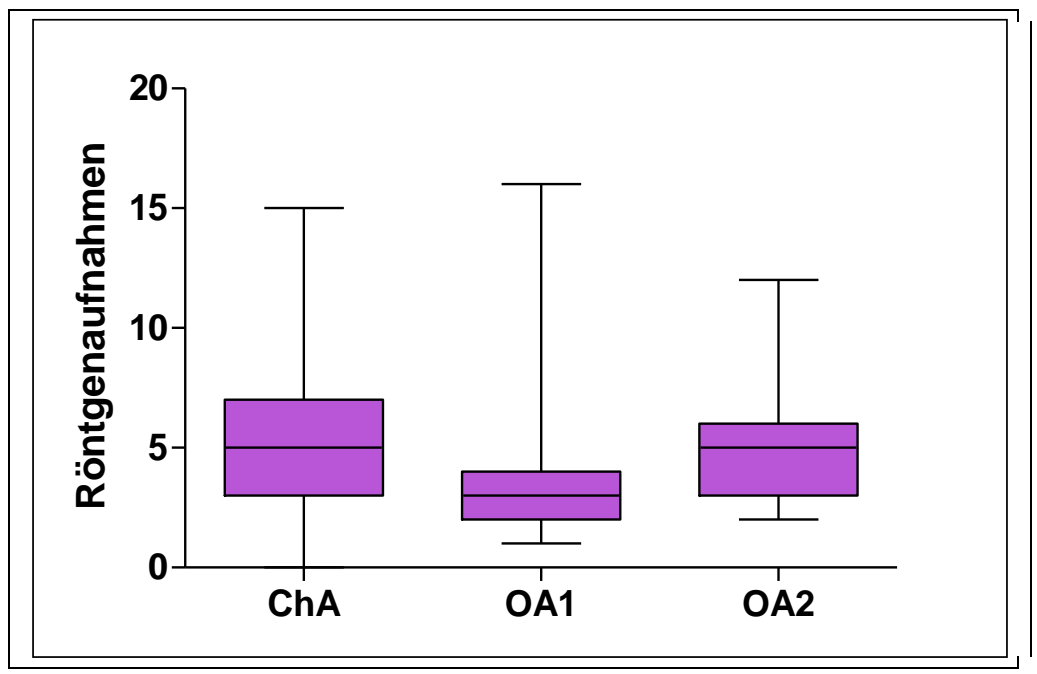

Zahl der intraoperativen Röntgenaufnahmen bei der ureterorenoskopischen Steinentfernung, aufgeschlüsselt nach Operateuren mit Facharztstatus. Die statistische Analyse zeigte eine niedrige Zahl von Röntgenaufnahmen bei Operationen durch den Operateur OA1, statistisch signifikant gegenüber ChA und OA2 ( $p=0,0008$ bzw. $p=0,0020$, t-Test). Der Vergleich von ChA und OA2 ergibt keine statistisch signifikanten Unterschiede ( $p=0,7479$, $t-$ Test). 


\section{Tabelle 9}

Zahl der intraoperativen Last-image-hold-Aufnahmen aufgeschlüsselt nach Operateuren mit Facharztstatus.

\begin{tabular}{|l|l|c|c|c|}
\hline Name & Arztstatus & Mittelwert & Minimum & Maximum \\
\hline ChA & Chefarzt & 2,2 & 0 & 12 \\
\hline OA1 & Oberarzt & 0,2 & 0 & 4 \\
\hline OA2 & Oberarzt & 0,4 & 0 & 12 \\
\hline
\end{tabular}

Abbildung 27

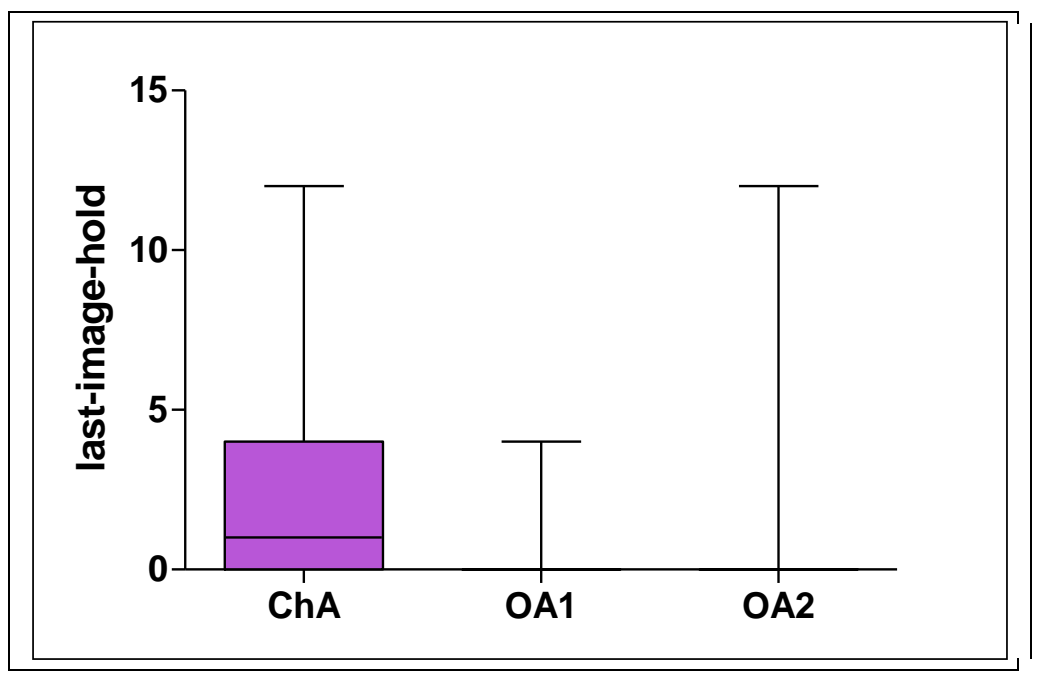

Zahl der intraoperativen Last-image-hold-Bilder bei der ureterorenoskopischen Steinentfernung, aufgeschlüsselt nach Operateuren mit Facharztstatus. Die statistische Analyse zeigte eine deutlich höhere Zahl von Last-image-hold-Bildern bei Operationen durch ChA (statistisch hochsignifikant gegenüber OA1 $(p=<$ 0,0001 , t-Test) und statistisch signifikant gegenüber OA2 ( $p=0,0004$, t-Test). Der Vergleich von OA1 und OA2 ergibt keine statistisch signifikanten Unterschiede ( $p$ $=0,4081, \mathrm{t}-$ Test $)$. 


\section{Diskussion}

Nachdem über zwei Jahrzehnte die extrakorporale Stoßwellenlithotripsie (ESWL) den Therapiestandard bei Nieren- und Harnleitersteinen darstellte, sind in den letzten Jahren zunehmend minimal-invasive endoskopische Therapieverfahren in den Vordergrund gerückt. Ein Grund hierfür ist vor allem die enorme technische Weiterentwicklung der Operationsendoskope, allen voran die Entwicklung kleinlumiger flexibler Ureterorenoskope, mit denen man inzwischen annähernd jeden Bereich des Nierenbeckenkelchsystems erreichen kann (Honeck et al. 2008). Parallel zur technischen Weiterentwicklung der Operationsendoskope kam es auch zur technischen Verfeinerung der Instrumente zur Steinbergung, die inzwischen durch die Flexibilität bis zu Nierenkelchsteinen vorgeführt werden können. Für größere Konkremente wurden die technischen Verfahren zur intrakorporalen Steindesintegration weiterentwickelt. Hervorzuheben ist hier die Entwicklung der LASER-Lithotripsie, bei der die LASER-Energie zur Steindesintegration über flexible Glasfasersonden mit geringem Sondendurchmesser im Mikrometerbereich auch durch flexible Ureterorenoskope bis zum Stein übertragen werden kann.

Der Weg der Ureterorenoskopie bis zur heutigen technischen Perfektion war dabei lang. Die erste dokumentierte Ureterorenoskopie wurde bereits 1912 durch Young bei einem 2jährigen Kind durchgeführt, bei dem er ein starres Kinderzystoskop mit einem Durchmesser von 9,5 Charr. durch einen stark erweiterten Harnleiter bis ins Nierenbecken führen konnte (Knoll und Alken 2006). 1960 stellte Marshall ein flexibles Fiberskop mit einem Durchmesser von 9 Charr vor (Marshall 1963, Reuter 2004). Takayasu et al. entwickelten 1968 dieses flexible Fiberskop so weiter, dass es neben der Fiberoptik auch über einen Operationskanal verfügte (Takagi et al. 1971). Goodman und Lyon führten 1975 die ersten ureterorenoskopischen Behandlungen von Tumoren im unteren Harnleiter durch (Goodman 1977, Lyon et al. 1979). Die Endoskopie des gesamten oberen Harntraktes bis zum Nierenbecken wurde aber erst durch die Entwicklung von langen Ureterorenoskopen durch Perez-Castro und Martinez-Pineiro (in Zusammenarbeit mit der Firma Karl Storz, Tuttlingen, Deutschland) ermöglicht (Perez-Castro und Martinez-Pineiro 1980). 
Nachdem zunächst durch die Ureterorenoskopie Steine im Harntrakt nur lokalisiert werden konnten, begann man schnell mit der Entfernung kleinerer Steine mittels Steinfasszangen und Steinfasskörbchen. Ein Problem stellten allerdings die größeren Harnleitersteine dar, die vor einer Steinbergung desintegriert werden mussten. Dies gelang zunächst mit Ultraschallsonden, die blind zum Stein geführt werden mussten, da sie nicht durch die Endoskope passten (Huffman et al. 1983). Durch die Entwicklung von Laserverfahren und dünneren Sonden war dann später eine visuell kontrollierte Lithotripsie möglich (Grasso 1996). Die Weiterentwicklung von Fiberoptiken mit dünnen Arbeits- und Spülkanälen ermöglichte schließlich die Herstellung von qualitativ deutlich besseren flexiblen Ureterorenoskopen. 1992 berichteten Abdel-Razzak und Bagley über die erfolgreiche flexible Ureterorenoskopie mit aktiv deflektierbaren Instrumenten (Abdel-Razzak und Bagley 1992). Technisch hochwertige, miniaturisierte, semirigide und flexible Ureterorenoskope mit einem Außendurchmesser von 7 - 9,5 Charr (in der Klinik für Urologie und Kinderurologie Bad Hersfeld: Außendurchmesser der semirigiden Instrumente 8 bzw. 9,5 Charr, Außendurchmesser der flexiblen Ureterorenoskope 7,5 Charr) sind heute Standard bei der Endoskopie des oberen Harntrakts und ermöglichen in Kombination mit Steinfasskörbchen und intrakorporalen Lithotripsietechniken wie der LASERLithotripsie die endoskopische Behandlung von Harnleiter- und Nierensteinen im gesamten oberen Harntrakt.

Die ureterorenoskopische Steinentfernung im Harnleiter- und Nierenbereich konkurriert dabei heute mit anderen Formen der minimal-invasiven Steinbehandlung wie der extrakorporalen Stoßwellenlithotripsie und der perkutanen Steinentfernung. Bei der Wahl der Therapieverfahren spielen dabei nicht nur Lage und Größe der Steine eine entscheidende Rolle, sondern zunehmend durch den Kostendruck im Gesundheitswesen und durch den Druck am Arbeitsmarkt auch Fragen der Behandlungsdauer und der rasch erzielbaren Steinfreiheitsraten. Eine medizinische Behandlung orientiert sich häufig an aktuellen Therapieleitlinien der unterschiedlichen medizinischen Fachgesellschaften. Nach der aktuellen S2-Leitlinie der Deutschen Gesellschaft für Urologie (www.urologenportal.de 2009) stellt die ureterorenoskopische Steinentfernung die Therapie der ersten Wahl bei distalen Harnleitersteinen $>1 \mathrm{~cm}$ dar. Im Bereich des proximalen Harnleiters ist die ESWL nach der noch gültigen Leitlinie Therapie der ersten Wahl, die Ureterorenoskopie die Therapie der zweiten Wahl. 
Bei den Nierenbeckensteinen und den Nierenkelchsteinen $<1 \mathrm{~cm}$ werden Ureterorenoskopie und extrakorporale Stoßwellenlithotripsie wieder als gleichwertig eingestuft, während für größere Steine die perkutane Litholapaxie und die ESWL als Therapie der ersten Wahl gelten (Tab.2).

Medizinische Leitlinien werden aufgrund der vorhandenen Datenlage erstellt und berücksichtigen so unterschiedliche Faktoren wie Verfügbarkeit von Therapieverfahren und Therapiemorbidität. Primäres Ziel der Steinbehandlung ist in erster Linie die Steinfreiheit bzw. die Rezidivfreiheit auch unter Berücksichtigung der notwendigen Therapiedauer und der erforderlichen Therapiewiederholungen (Therapieeffizienz).

Unter diesen Aspekten ist die ureterorenoskopische Steinentfernung als Therapie der Wahl bei distalen Harnleitersteinen unumstritten. Hier liegen die Steinfreiheitsraten bei einer Steingröße unter $5 \mathrm{~mm}$ zwischen $89 \%$ und $100 \%$, bei Steinen größer als $10 \mathrm{~mm}$ zwischen $73 \%$ und $98 \%$ (Hofmann 2006). Die ESWL weist hier deutlich geringere Steinfreiheitsraten zwischen $45 \%$ und $98 \%$ auf. Eine erneute Behandlung ist bei der Ureterorenoskopie in 0 - $4 \%$ aller distalen Harnleitersteine erforderlich, bei der ESWL bei ca. $20 \%$ der Patienten (Peschel et al. 1999).

Unterschiedlich ist die Bewertung der Ureterorenoskopie zur Therapie der proximalen Harnleitersteine. In älteren Arbeiten werden Steinfreiheitsraten bei der Ureterorenoskopie von 62 - 98 \% beschrieben (Mobley et al. 1993). Differenziert man nach Steingröße, so zeigen aber neuere Arbeiten für Steine unter $1 \mathrm{~cm}$ Größe Steinfreiheitsraten von bis zu $100 \%$, für Steine über $1 \mathrm{~cm}$ Größe von immer noch 88 $\%$, während die vergleichbaren Steinfreiheitsraten für die ESWL mit 80 \% für Steine unter $1 \mathrm{~cm}$ und mit $60 \%$ für Steine über $1 \mathrm{~cm}$ angegeben werden (Salem 2009). Ähnliche Steinfreiheitsraten wurden von Tawfick im Jahr 2010 beschrieben, mit Steinfreiheitsraten im oberen Harnleiter für die URS von $94 \%$ verglichen mit $58 \%$ bei der ESWL (Tawfick 2010).

Diese verbesserten Therapieergebnisse der Ureterorenoskopie sind auf die gerätetechnische Weiterentwicklung der letzten Jahre zurückzuführen. Allerdings haben diese jüngsten Entwicklungen und die daraus resultierende Therapieverbesserung noch nicht Eingang in die jeweiligen Therapieleitlinien der urologischen Fachgesellschaften gefunden. 
Es ist aber damit zu rechnen, dass unter dem Eindruck der verbesserten Therapieergebnisse die Ureterorenoskopie auch Leitlinienstandard in der Therapie des proximalen Harnleitersteines werden wird, ein Standard, der an der Klinik für Urologie und Kinderurologie des Klinikum Bad Hersfeld aufgrund der verfügbaren Ureterorenoskope und der Möglichkeit der intrakorporalen LASER-Lithotripsie bereits jetzt erreicht ist.

Für Harnsteine im Bereich des Nierenbeckens und der Nierenkelche steht neben der Ureterorenoskopie und der ESWL auch die perkutane Litholapaxie als mögliche Therapieform zur Verfügung. Für Nierenkelch- und Nierenbeckensteine $<2 \mathrm{~cm}$ Größe zeigen jüngste Publikationen für die Ureterorenoskopie, ggf. in Kombination mit der LASER-Lithotripsie, Steinfreiheitsraten von $77 \%$ bis $90 \%$ (Hussain et al. 2010, Cansino Alcaide et al. 2010). Vergleichbare Therapieergebnisse werden mit der perkutanen Litholapaxie (PCNL) erreicht (Chung et al. 2008). In den letzten Jahren wurde die PCNL zur minimal-invasiven perkutanen Nephrolitholapaxie (MiniPCNL) mit kleineren perkutanen Ports weiterentwickelt. Mit der Mini-PCNL wird, in Kombination mit der intrakorporalen Lithotripsie z.B. mit der LASER-Lithotripsie, über Steinfreiheitsraten zwischen $90 \%$ und 92,9 \% berichtet (Nagele et al. 2008b, Lahme et al. 2008).

Bei der extrakorporalen Stoßwellenlithotripsie (ESWL) variiert die Steinfreiheitsrate in Abhängigkeit von Steingröße und Lage des Steines in der Niere. Bei Steinlage im Nierenbecken sowie in mittleren und oberen Nierenkelchgruppen können Steinfreiheitsraten von $70 \%$ bis $90 \%$ erzielt werden (Schmidt und Pflüger 2006). Für untere Nierenkelchsteine sind die Steinfreiheitsraten durch ESWL deutlich schlechter. Hier werden Steinfreiheitsrates je nach Steingröße zwischen $14 \%$ und $74 \%$ erreicht, wobei bei größeren Steinen die Steinfreiheitsrate noch einmal deutlich abfällt (Nagele et al. 2008a). Das Problem der unteren Kelchsteine liegt darin, dass nach erfolgreicher Desintegration die Steinfragmente gegen die Schwerkraft aus der unteren Kelchgruppe über das Nierenbecken in den Harnleiter eintreten müssen, was nur bedingt der Fall ist.

Für größere Nierensteine $>2 \mathrm{~cm}$ Größe und Nierenbeckenausgusssteine gilt die (Mini-)PCNL als Therapie der ersten Wahl. 
Aufgrund der jüngsten Publikationen und aufgrund der eigenen Erfahrungen stellt in der Klinik für Urologie und Kinderurologie des Klinikums Bad Hersfeld - anders als in den noch gültigen Leitlinien der Deutschen Gesellschaft für Urologie aus dem Jahr 2009 - die Ureterorenoskopie die Therapie der Wahl bei weitgehend allen Steinen im Harnleiter und bei Steinen im Nierenbecken und in den Nierenkelchen bis zu einer Größe von $2 \mathrm{~cm}$ dar. Bei Nierensteinen größer als $2 \mathrm{~cm}$ gilt bei uns die Mini-PCNL als Therapie der ersten Wahl. Die ESWL wird nur noch in seltenen ausgesuchten Fällen, zum Beispiel bei erhöhtem Narkoserisiko, bei Lagerungsproblemen oder auf ausdrücklichen Wunsch des Patienten durchgeführt.

Die Therapieeffizienz muss in Relation zu den therapieimmanenten Komplikationsmöglichkeiten gesehen werden. Für die ureterorenoskopische Steinentfernung werden in der Literatur Komplikationsraten von bis $9-11 \%$ angegeben (Tab.11). Die wichtigsten Komplikationen stellen dabei Schleimhautverletzungen oder penetrierende Wandverletzungen im Harnleiter dar. Harnleiterverletzungen können zur postoperativen Harnstauung mit entsprechender obstruktiver Symptomatik mit Schmerzen oder Fieber führen. Intraoperative Harnleiterverletzungen machen deshalb die vorübergehende Einlage einer inneren Harnleiterschiene für die postoperative Phase erforderlich. Die Gefahr von Harnleiterverletzungen nimmt mit der Zahl der Gerätepassagen durch den Harnleiter zu, z.B. wenn multiple, scharfkantige Konkremente geborgen werden müssen. Vorbeugend kann deshalb bei häufiger Passage des Endoskopes z.B. durch das Harnleiterostium eine intraoperative Ureterschutzhülse („Schleuse“) eingelegt werden. Eine traumatisch bedingte Harnleiterstriktur als Langzeitfolge ist heute bei Verwendung moderner Ureterorenoskope mit einer Häufigkeit von ca. 1 \% selten (Hofmann 2006). Die Miniaturisierung der Ureterorenoskope wird zu einer weiteren Senkung der gerätebedingten Harnleiterverletzungen führen. Eingriffsbedingt sind auch Komplikationen wie eine postoperative Hämaturie oder ein iatrogener Harnwegsinfekt. Als schwerste Komplikationen der Ureterorenoskopie sind als Einzelkasuistiken Harnleiterabrisse (Brandt et al. 2010), arteriovenöse Fistelbildungen (Tiplitzky et al. 2007) oder auch subkapsuläre Nierenhämatome (Bansal et al. 2010) beschrieben. 


\section{Tabelle 11}

Häufige Komplikationen bei ureterorenoskopischer Steinentfernung in Abhängigkeit von der Steinlage (Preminger et al. 2007).

\begin{tabular}{|l|c|c|c|}
\hline & $\begin{array}{c}\text { Unteres } \\
\text { Harnleiterdrittel }\end{array}$ & $\begin{array}{c}\text { Mittleres } \\
\text { Harnleiterdrittel }\end{array}$ & $\begin{array}{c}\text { Oberes } \\
\text { Harnleiterdrittel }\end{array}$ \\
\hline Harnwegsinfekt & $2-7 \%$ & $0-7 \%$ & $1-8 \%$ \\
\hline Sepsis & $1-11 \%$ & $1-11 \%$ & $2-6 \%$ \\
\hline Harnleiterverletzung & $3-9 \%$ & $3-8 \%$ & $3-9 \%$ \\
\hline Harnleiterstriktur & $1-2 \%$ & $2-7 \%$ & $1-5 \%$ \\
\hline
\end{tabular}

Während sich in der Literatur zahlreiche Publikationen zur Machbarkeit ureterorenoskopischer Eingriffe und den eingriffstypischen Komplikationen finden, gibt es kaum Arbeiten zur Strahlenbelastung während einer ureterorenokopischen Steinbehandlung.

Einige Untersuchungen befassten sich mit der Strahlenbelastung der Operateure und des Pflegepersonals bzw. des anästhesiologischen Personals während der Durchleuchtung bei ureterorenoskopischen Eingriffen. Bei diesen Untersuchungen wurden intraoperativ mobile Durchleuchtungseinheiten (C-Bögen) benutzt und die Strahlenbelastung mittels Dosimetern an unterschiedlichen Körperregionen des ärztlichen und assistierenden Personals gemessen. Die höchsten Dosen traten bei komplexen Eingriffen im Bereich der Nieren auf, wobei die Strahlenbelastung erwartungsgemäß mit der Durchleuchtungszeit korrelierte.

Hellawell et al. 2005 untersuchten die Strahlenbelastung im Bereich der Extremitäten und der Augen der Operateure und des assistierenden Personals während diagnostischer und therapeutischer Ureterorenoskopien am Harnleiter. Sie verglichen die erhobenen Daten mit der Strahlenbelastung bei der Durchführung einer PCNL. Die durchschnittliche Strahlenbelastung war bei der PCNL an allen gemessenen Körperpunkten deutlich höher als bei der URS. 
Bei der URS wurde für den Operateur die höchste Strahlenbelastung mit durchschnittlich 11,6 $\mu$ Gy im Bereich der Oberschenkels (167 $\mu$ Gy bei der PCNL), die niedrigste mit durchschnittlich 1,9 $\mu \mathrm{Gy}$ im Bereich des Kopfes (40 $\mu \mathrm{Gy}$ bei der PCNL) gemessen. Die instrumentierende Schwester, die weiter entfernt von der Strahlenquelle stand, war mit durchschnittlich 0,8 $\mu \mathrm{Gy}$ im Bereich der Oberschenkel (11,0 $\mu$ Gy bei der PCNL) und durchschnittlich 0,8 $\mu$ Gy im Bereich des Kopfes (16 $\mu G y$ bei der PCNL) einer deutlich geringeren Strahlenbelastung ausgesetzt (Hellawell et al. 2005). Die Schlussfolgerungen aus diesen Untersuchungen entsprechen den gängigen Strahlenschutzrichtlinien zum Schutz des Personals wie das Tragen von adäquater Schutzkleidung, die möglichst kurze Strahlenexposition und entsprechend des Abstand-Quadrat-Gesetzes die Einhaltung eines größtmöglichen Abstandes von der Strahlenquelle.

Die Strahlenbelastung für Patienten während eines ureterorenoskopischen Eingriffes wurde von Krupp et al. in Abhängigkeit von Geschlecht und Body-Mass-Index (BMI) gemessen. Diese Untersuchungen wurden an acht Leichen durchgeführt, bei denen eine linksseitige Ureterorenoskopie durchgeführt wurde, und die dabei über einen identischen Zeitraum hinweg geröntgt wurden. Neben der automatisch am Röntgengerät gemessenen Gesamtstrahlendosis wurde zusätzlich mittels Dosimeter die Ortsdosis an unterschiedlichen Körperregionen gemessen. Mit Ausnahme der Gonadenbelastung war die Strahlenbelastung nicht vom Geschlecht abhängig. Bezüglich der gonadalen Strahlenexposition betrug die durchschnittlichen Strahlenbelastung der linken Gonade beim Mann 0,36 mGy, bei der Frau 3,4 mGy und die Strahlenbelastung der rechten Gonade beim Mann 0,39 mGy, bei der Frau 1,88 mGy. Diese Unterschiede sind durch die unterschiedliche topographische Lage der Gonaden zum linken Harnleiter zu erklären. Eine tendenziell erhöhte Strahlenbelastung bestand abhängig vom Body-Mass-Index im Hautbereich hinter der behandelten Niere. Diese betrug bei einem BMI $<30$ durchschnittlich 8,39 mGy, bei einem BMI > 30 durchschnittlich 13,98 mGy; dieser Unterschied war allerdings statistisch nicht signifikant. Untersuchungen differenziert nach Steingröße, Steinlage, Operateur, Durchleuchtungszeit, Anfertigung von Röntgenaufnahmen oder Komplexizität des Eingriffes wurden nicht durchgeführt (Krupp et al. 2010). 
Untersuchungen zur Strahlenbelastung der Patienten während ureterorenoskopischer Eingriffe wurden 1990 von Bagley und Cubler-Goodman publiziert. Sie verglichen die Strahlenbelastung durch die Anwendung eines (zum damaligen Zeitpunkt konventionellen und nicht digitalisierten) Röntgen-C-Bogens mit kontinuierlichem Durchleuchtungsbild mit einem C-Bogen mit gepulster Durchleuchtung. Die Messung der Strahlenbelastung erfolgte dabei mittels Ortsdosimetern an unterschiedlichen Körperstellen der Patienten (z.B. Rippenbogen, Sakroiliakalbereich). Die durchschnittliche effektive Durchleuchtungszeit betrug bei kontinuierlicher Durchleuchtung 2,0 bis 4,7 Minuten, bei gepulster Durchleuchtung 0,30 bis 0,63 Minuten, mit den kürzesten Durchleuchtungszeiten bei der diagnostischen Ureterorenoskopie mit semirigiden Geräten und den längsten Durchleuchtungszeiten bei der ureterorenoskopischen Steinentfernung mittels flexiblen Ureterorenoskopen bzw. bei der Kombination aus semirigider und flexibler Ureterorenoskopie. Durch diese Durchleuchtungszeiten resultierte bei ureterorenoskopischen Eingriffen am distalen Ureter eine Ortsdosisbelastung sakroiliakal von ca. 3,0 mSv bei kontinuierlicher Durchleuchtung bzw. 0,39 mSv bei gepulster Durchleuchtung. Bei ureterorenoskopischen Eingriffen an der Niere oder im oberen Harnleiter betrug die gemessene Ortsdosis am Rippenbogen ca. 0,97 mSv bei kontinuierlicher Durchleuchtung und $0,29 \mathrm{mSv}$ bei gepulster Durchleuchtung. Die Untersuchung zeigte damit, dass durch eine gepulste Durchleuchtung während ureterorenoskopischer Eingriffe die Strahlenbelastung der Patienten signifikant gesenkt werden kann. Die gepulste Durchleuchtung ist heute Standard in digitalisierten Röntgenanlagen; so wird in der Röntgenanlage Uroskop Access das Durchleuchtungsbild aus 25 Einzelbilder / Sekunde generiert. Die Untersuchung zeigte aber auch, dass die Durchleuchtungszeit und damit die Strahlenbelastung wesentlich von der Art des Eingriffs (diagnostisch versus therapeutisch) und von der Art der verwendeten Endoskope (semirigide versus flexible Ureterorenoskope) abhängt (Bagley und Cubler-Goodman 1990).

In unserer Arbeit wurde die Höhe der Strahlenbelastung bei der Ureterorenoskopie mit modernstem Instrumentarium unter Verwendung digitaler Röntgenanlagen nach unterschiedlichen Kriterien wie Art der Ureterorenoskopie (semirigide versus flexible URS), Lage der Steine (Harnleiter versus Niere), Größe der Steine, Art der Steinentfernung (einfache Steinextraktion versus LASER-Lithotripsie), Länge der Durchleuchtungszeit, Anzahl der Röntgenaufnahmen während des Eingriffes und Notwendigkeit der erneuten Harnleiterschienung ermittelt. 
Das Ziel war, daraus Optimierungsmöglichkeiten, d.h. heißt Möglichkeiten zur Reduktion der Strahlenbelastung bei der ureterorenoskopischen Steinbehandlung, abzuleiten. Sekundärer Untersuchungsparameter war die Frage, ob Unterschiede zwischen einzelnen Operateuren bestehen. Dazu wurden in einer retrospektiven Analyse die Strahlenbelastung bei 215 Patienten ermittelt, die wegen eines oder wegen mehrerer Steine im Harnleiter und/oder in der Niere interventionell mittels ureterorenoskopischer Steinentfernung behandelt wurden.

Die Strahlenbelastung wurde dabei als Dosis-Flächen-Produkt ermittelt. Dieses Dosis-Flächen-Produkt bietet den Vorteil, dass es sofort am Röntgengerät gemessen bzw. abgelesen werden kann. Andere Dosisbegriffe wie die effektive Dosis, die Äquivalenzdosis oder die Energiedosis sind nur mittels komplexer Messverfahren und Konversionsfaktoren zu bestimmen und somit für den Routinebetrieb nicht gut geeignet. Die Messung des Dosis-Flächen-Produktes erfolgt mit einer dauernd im Strahlengang befindlichen flachen lonisationskammer und ist ein Produkt aus Dosisleistung (Gy/s), Zeit (s) und Feldgröße $\left(\mathrm{cm}^{2}\right)$. Diese Messkammer im Strahlengang ist heute aufgrund der nach §28 der Röntgenverordnung aus dem Jahr 2003 zwingend vorgeschriebenen Dokumentation der Strahlenexposition regelhaft in Röntgenanlagen integriert. Die Messung des Dosis-Flächen-Produkts durch lonisationskammern im Strahlengang bietet die Möglichkeit einer objektiven Vergleichbarkeit bei Untersuchungen zur Strahlenexposition, da die Strahlenbelastung unabhängig von möglicherweise unterschiedlich positionierten Ortsdosimetern mit der daraus resultierenden eingeschränkten Vergleichbarkeit der erhobenen Daten gemessen werden kann.

Zusammenfassend zeigen unsere Untersuchungen eine signifikant höhere Strahlenbelastung bei der Behandlung von Nierensteinen gegenüber der Behandlung von Harnleitersteinen, mit einer weiteren signifikanten bis hochsignifikanten Steigerung der Strahlenbelastung bei der simultanen Behandlung von Nieren- und Harnleitersteinen (durchschnittlich 270,9 $\mu \mathrm{Gy}^{*} \mathrm{~m}^{2}$ bei Harnleitersteinen, $340,1 \mu \mathrm{Gy}^{*} \mathrm{~m}^{2}$ bei Nierensteinen bzw. $744,2 \mu \mathrm{Gy}^{*} \mathrm{~m}^{2}$ bei simultaner Behandlung von Nieren- und Harnleitersteinen). 
Auch operationstechnische Unterschiede bei der Durchführung der Ureterorenoskopie hatten unterschiedliche Strahlenbelastungen zur Folge. Wie ebenfalls von anderen Autoren beobachtet ging die Verwendung der technisch etwas aufwändigeren flexiblen Ureterorenoskope (z.B. regelhaft verbunden mit der Verwendung von Führungsdrähten) mit einer signifikant höheren Strahlenbelastung einher (durchschnittlich $221,9 \mu \mathrm{Gy}{ }^{*} \mathrm{~m}^{2}$ bei semirigider Ureterorenoskopie und $390,4 \mu \mathrm{Gy}^{*} \mathrm{~m}^{2}$ bei flexibler Ureterorenoskopie). Erwartungsgemäß führte die Notwendigkeit einer LASER-Lithotripsie zu einer erhöhten Strahlenbelastung verglichen mit der einfachen Steinbergung mittels Dormiakörbchen $\left(575,5 \mu \mathrm{Gy}{ }^{*} \mathrm{~m}^{2}\right.$ versus $\left.297,4 \mu \mathrm{Gy}{ }^{*} \mathrm{~m}^{2}\right)$. Ebenso wie die LASER-Lithotripsie ist auch die Notwendigkeit einer postoperativen Harnleiterschienung Ausdruck eines technisch komplexeren Eingriffes und war damit erwartungsgemäß mit einer höheren Strahlenbelastung assoziiert $\left(267,9 \mu \mathrm{Gy}^{*} \mathrm{~m}^{2}\right.$ ohne Harnleiterschienung, $831,4 \mu \mathrm{Gy}^{*} \mathrm{~m}^{2}$ bei postoperativer Harnleiterschienung).

Dabei korrelierte die Höhe der Strahlenbelastung während eines Eingriffs erstaunlicher Weise nur gering mit der Anzahl der angefertigten Röntgenaufnahmen, aber deutlich mit der Durchleuchtungszeit.

Die mittels Dosis-Flächen-Produkt ermittelte Strahlenbelastung ist nur bedingt auf die effektive Dosis übertragbar, die in anderen Untersuchungen zur Dokumentation der Strahlenbelastung herangezogen wurde. Abhängig von der Körperregion kann aber über Konversionsfaktoren aus dem Dosis-Flächen-Produkt die effektive Dosis abgeleitet werden. Zur Berechnung der effektiven Dosis im Abdomen gilt ein Konversionsfaktor von $0,323 \mathrm{mSv} /\left(\mathrm{Gy}^{*} \mathrm{~cm}^{2}\right)$ (Bernhardt at al. 1995). Zur besseren Vergleichbarkeit unserer Daten mit den Daten der Literatur ist die von uns mittels Dosis-Flächen-Produkt gemessene Strahlenbelastung in Tabelle 12 als abdominelle effektive Dosis umgerechnet dargestellt.

Die Strahlenbelastung bei der ureterorenoskopischen Steinentfernung muss im Kontext der Strahlenbelastung bei Diagnostik und Therapie von Harnleiter- und Nierensteinen gesehen werden. Eine konventionelle Abdomenübersichtsaufnahme (Nierenleeraufnahme) in Form der klassischen Film-Folien-Kombination resultiert in einer Strahlenbelastung von ca. 0,5 mSv. 


\section{Tabelle 12}

Mittlere Strahlenbelastung gemessen als Dosis-Flächen-Produkt und errechnete effektive abdominelle Dosis (Konversionsfaktor 0,323 mSv / $\mathrm{Gy}^{*} \mathrm{~cm}^{2}=0,00323 \mathrm{mSv} /$ $\left.\mu \mathrm{Gy}^{*} \mathrm{~m}^{2}\right)$.

\begin{tabular}{|l|c|c|}
\hline & $\begin{array}{c}\text { Dosis-Flächen- } \\
\text { Produkt }\left(\mu \mathrm{Gy}^{*} \mathrm{~m}^{2}\right)\end{array}$ & $\begin{array}{c}\text { Effektive abdominelle } \\
\text { Dosis }(\mathrm{mSV})\end{array}$ \\
\hline URS bei Harnleitersteinen & 271,6 & 0,81 \\
\hline URS bei Nieresteinen & 340,1 & 1,02 \\
\hline URS bei Niere- und Harnleitersteinen & 628,0 & 1,88 \\
\hline Semirigide URS & 221,9 & 0,67 \\
\hline Flexible URS & 324,4 & 0,97 \\
\hline URS ohne Lithotripsie & 297,4 & 0,89 \\
\hline URS mit LASER-Lithotripsie & 575,5 & 1,73 \\
\hline
\end{tabular}

Das konventionelle Ausscheidungsurogramm in Film-Folien-Kombinationen, das bis vor kurzem den Standard in der Diagnostik von Harnsteinen darstellte, führt durch Leeraufnahme und in der Regel zwei Röntgenaufnahmen nach Kontrastmittelgabe zu einer Strahlenbelastung von ca. 1,4-1,5 mSv (Thomson et al. 2001). Die Strahlenbelastung erhöht sich entsprechend, wenn zur exakten Diagnostik weitere Aufnahmen wie Spätaufnahmen oder ein Tomogramm notwendig werden.

Die deutlich höhere Sensitivität und Spezifität der Computertomographie führte jedoch dazu, dass heute das Nativ-CT das Diagnostikverfahren der Wahl darstellt. Allerdings ist das Nativ-CT mit einer deutlich höheren Strahlenbelastung von 2,8 - 5,0 mSv assoziiert (Thomson et al. 2001). Durch technische Modifikationen wie der Durchführung eines Ultraniedrig-CTs kann bei gleich bleibender Sensitivität und Spezifität die Strahlenbelastung auf 1,0-2,2 mSv reduziert werden (Knopfle et al. 2003). Am Klinikum Bad Hersfeld sind Ultraniedrig-CT-Untersuchungen (low-doseCT) seit dem März 2010 möglich. 
Nach eigenen Messungen konnte durch diese Implementierung moderner CTTechniken eine Reduktion der Strahlenbelastung von durchschnittlich 5,0 - 6,0 mSv auf 1,8-2,2 mSv erreicht werden.

Die Strahlenbelastung bei den alternativen Therapieverfahren ESWL und PCNL ist in der Literatur mehrfach untersucht worden. Bei Anwendung der ESWL werden bei radiologischer Steinortung abhängig von der Steingröße, Steinposition und Dauer des Eingriffes Strahlenbelastungen von 0,87 bis 1,63 mSv beschrieben (Sandilos et al. 2006). Bei der sonografischen Steinortung fällt in der Regel keine intraoperative Strahlenbelastung an. Allerdings muss die Therapieeffektivität gerade bei der ESWL durch postoperative radiologische Kontrollen überprüft werden, um den suffizienten Steinabgang und die Steinfreiheit zu dokumentieren. Die unmittelbaren präoperativen und postoperativen radiologischen Kontrollen können die Gesamtstrahlenbelastung bei der ESWL verdoppeln (Talati et al. 2000). Untersuchungen zur Strahlenbelastung bis zum Erreichen der dokumentierten Steinfreiheit nach ESWL finden sich in der Literatur nicht. Es muss damit gerechnet werden, dass durch die Zahl der im postoperativen Verlauf notwendigen Nierenleeraufnahmen mit jeweils ca. 0,5 mSv/ Aufnahme die Strahlenbelastung bei der Steinbehandlung mittels ESWL letztendlich deutlich höher liegt als in den publizierten Daten zur unmittelbaren intraoperativen Strahlenbelastung.

Die perkutane Steinentfernung (PCNL bzw. Mini-PCNL), die vor allen Dingen bei größeren Nierenbeckensteinen als Standardtherapieverfahren gilt, geht mit einer deutlichen intraoperativen Strahlenbelastung für den Patienten einher. Auch bei der PCNL ist die Höhe der Strahlenbelastung von verschiedenen Faktoren wie BodyMass-Index, Steingröße und Komplexizität des Eingriffes abhängig (Mancini et al. 2010). Die durchschnittliche Strahlenbelastung beträgt in der Literatur zwischen 0,59 $\mathrm{mSv}$ und 10,2 mSv bzw. ca. $406 \mathrm{cGy} / \mathrm{cm}^{2}$. Alle Autoren berichten über eine deutliche Abhängigkeit der Strahlenbelastung von der Durchleuchtungszeit und der Länge des Eingriffes (Kumar 2008, Safak et al. 2009, Mancini et al. 2010). 
Unsere Untersuchungen zur intraoperativen Strahlenbelastung zeigen, dass es sich bei der ureterorenoskopischen Steinentfernung unter Verwendung moderner digitaler Röntgenarbeitsplätze um ein Operationsverfahren mit nur geringer intraoperativer und (bei unmittelbar erreichbarer Steinfreiheit) fehlender postoperativer Strahlenbelastung handelt, verglichen mit der Strahlenbelastung bei der Diagnostik von Harnleiter- und Nierensteinen und bei der Therapie mittels ESWL und PCNL.

Interessant war in unserer Untersuchung dabei die Auswertung der Strahlenbelastung hinsichtlich der einzelnen Operateure, die insbesondere bei erfahrenen Operateuren mit Facharztstatus deutliche Unterschiede zeigte. So betrug die durchschnittliche Strahlenbelastung 432,4 $\mu \mathrm{Gy}^{*} \mathrm{~m}^{2}$, $194,1 \mu \mathrm{Gy}^{*} \mathrm{~m}^{2}$ bzw. 538,1 $\mu G y^{*} m^{2}$. Diese Strahlenbelastung korrelierte mit Unterschieden bei der Durchleuchtungszeit und Zahl der intraoperativen Röntgendokumentation, sei es als Röntgenbild oder als Last-image-hold-Aufnahme. Hinter diesen Zahlen verbergen sich im Wesentlichen die unterschiedlichen Präferenzen der Operateure hinsichtlich der justiziablen Dokumentation der wichtigsten intraoperativen Schritte.

Der Aspekt des Operateurs war bisher nicht Gegenstand von Untersuchungen zur Strahlenbelastung bei der operativen Steinsanierung und wurde bisher nicht in der Literatur diskutiert. Unsere Ergebnisse zeigen aber, dass der Operateur wesentlichen Einfluss auf die intraoperative Strahlenbelastung hat. Neben der technischen Weiterentwicklung sowohl in der Gerätetechnik als auch im Strahlenschutz kommt deshalb dem Faktor Operateur eine wesentliche Rolle im Strahlenschutz von Patient aber auch des behandelnden Personals im Operationssaal zu.

Die Etablierung eines standardisierten operativen Vorgehens mit Festlegung der zu dokumentierenden OP-Schritte, die bewusste Reduktion der intraoperativen Durchleuchtungszeit und der Verzicht von Röntgenaufnahmen zu Gunsten der Last-imagehold-Aufnahmen (bei etwas schlechterer Bildqualität) können dazu beitragen, die intraoperative Strahlenbelastung bei der ureterorenoskopischen Steinentfernung noch weiter zu reduzieren.

Gemessen an der Therapieeffizienz und an der minimalen Morbidität auch hinsichtlich der intraoperativen Strahlenbelastung stellt damit die ureterorenoskopische Steinentfernung aus unserer Sicht die Therapie der Wahl bei Harnleitersteinen und bei Nierensteinen bis zu einer Größe von $2 \mathrm{~cm}$ dar. 


\section{Zusammenfassung}

Die Ureterorenoskopie zur Harnsteinbehandlung stell ein minimal-invasives Therapieverfahren mit hoher Steinfreiheitsrate, nicht nur im distalen Harnleiterdrittel, dar. Unseres Erachtens ist sie die Therapie der Wahl bei distalen und proximalen Harnleitersteinen sowie auch von Nierensteinen bis zu einer Größe von $2 \mathrm{~cm}$.

In dieser Arbeit wurde die Strahlenbelastung bei der Ureterorenoskopie hinsichtlich unterschiedlicher Kriterien untersucht. Es wurde dabei ein Kollektiv von 215 Patienten erfasst, die wegen eines oder mehrerer Steine im Zeitraum zwischen 01.07.2008 und 01.07.2010 interventionell mittels Ureterorenoskopie behandelt wurden.

Die Untersuchungen zeigten dabei zusammenfassend eine signifikante Zunahme der Strahlenbelastung mit zunehmender Komplexizität des Eingriffs (signifikant höhere Strahlenbelastung bei der Behandlung von Nierensteinen gegenüber der Behandlung von Harnleitersteinen; signifikant bis hochsignifikant höhere Strahlenbelastung bei der simultanen Behandlung von Nieren- und Harnleitersteinen; signifikant höhere Strahlenbelastung beim Einsatz flexibler Ureterorenoskope; signifikant höhere Strahlenbelastung bei der Notwendigkeit der intrakorporalen LASER-Lithotripsie und der postoperativen Harnleiterschienung).

Es bestand eine enge Korrelation von Durchleuchtungszeit und Strahlenbelastung, während die Anzahl der angefertigten Röntgenaufnahmen und die Steingröße nur gering mit der Strahlenbelastung korrelierten.

Es fanden sich statistisch signifikante Unterschiede in der Strahlenbelastung in Abhängigkeit vom Operateur, die sich in unterschiedlichen intraoperativen Durchleuchtungszeiten und intraoperativen Röntgendokumentationen mittels Röntgenaufnahmen oder Last-image-hold-Aufnahmen widerspiegeln. 
Unsere Daten zeigen damit, dass neben der Komplexizität des Eingriffes gemessen an der Art der Ureterorenoskopie (semirigide oder flexibel), der Art der Steinbergung (ohne und mit LASER-Lithotripsie) und der Notwendigkeit der postoperativen Harnleiterschienung der Operateur wesentlichen Einfluss auf die Strahlenbelastung bei der Ureterorenoskopie hat. Neben der Reduktion der intraoperativen Durchleuchtungszeiten und dem Verzicht von Röntgenaufnahmen zu Gunsten von Last-image-hold-Bildern kann aus unserer Sicht insbesondere die Etablierung abteilungseinheitlicher Vorgehensweisen mit Festlegung der justiziabel zu dokumentierenden Operationsschritte dazu beitragen, die Strahlenbelastung bei der ureterorenoskopischen Steinentfernung weiter zu senken. 


\section{Literaturverzeichnis}

Abdel-Razzak OM, Bagley DH (1992): Clinical experience with flexible ureterorenoscopy. J Urol $\underline{148}, 1788-1792$

Albala DM, Assimos DG, Clayman RV, Denstedt JD, Grasso M, Gutierrez-Aceves J, Kahn RI, Leveillee RJ, Lingeman JE, Macaluso JN Jr. (2001): Lower pole I: a prospective randomized trial of extracorporeal shock wave lithotripsy and percutaneous nephrolithotomy for lower pole nephrolithiasis - initial results. J Urol $\underline{166}, 2072-2080$

Bagley DH, Cubler-Goodman A (1990): Radiation exposure during ureteroscopy. J Urol 144, 1356-1358

Bansal U, Sawant A, Dhabalia J (2010): Subcapsular renal haematoma after ureterorenoscopy: an unknown complication of a known procedure. Urol Ann $\underline{2}$, 119121

Bernhardt JU, Veit R, Bauer, A (1995): Erhebung zur Strahlenexposition der Patienten bei der Röntgendiagnostik. Z Med Phys $\underline{5}$, 33-39

Brandt AS, von Rundstedt FC, Lazica DA, Roth S (2010): Harnleiterrekonstruktion nach ureterorenoskopischer Verletzung. Urologe 49, 812-821

Cansino Alcaide JR, Reinoso Elbers J, López Sánchez D, Perez Gonzales S, Agulera Bazan A, Rando Tous A, Hidolgo Togores L, De la Pena Barthel J (2010): Flexible ureterorenoscopy (URS): technique and results. Arch Esp Urol $\underline{63}, 862-870$

Chowdhury FU, Kotwal S, Raghunathan G, Wah TM, Joyce A, Irving HC (2007): Unenhanced multidetector CT (CT KUB) in the initial imaging of suspected acute renal colic: evaluating a new service. Clin Radiol $\underline{62}, 970-977$

Chung BI, Aron M, Hegarty NJ, Desai MM (2008): Ureterorenoscopic versus percutaneous treatment for medium-size $(1-2-\mathrm{cm})$ renal calculi. J Endourol $\underline{22}$, 343346

Esen T, Alken P: Harnsteinerkrankung; in: Kinderurologie in Klinik und Praxis; hrsg. v. Thüroff JW, Schulte Wissermann H; Thieme Verlag, Stuttgart 2000, 424-445 
Fahlenkamp D, Noack B, Lebentrau S, Belz H (2008): Urolithiasis bei Kindern. Urologe 47, 545-555

Goodman TM (1977): Ureteroscopy with pediatric cystoscope in adult. Urology $\underline{9}, 394$

Grasso M (1996): Experience with the holmium laser as an endoscopic lithotrite. Urology $\underline{48}, 199-206$

Hellawell GO, Mutch SJ, Thevendran G, Wells E, Morgan RJ (2005): Radiation exposure and the urologist: what are the risks? J Urol 174, 948-952

Hesse A (2002): Harnsteine Teil 1: Epidemiologie, Labordiagnostik, Genetik und Infekte. Urologe A $\underline{41}, 496-505$

Hofmann R(2006): Ureterorenoskopie (URS) bei Harnleitersteinen. Urologe $\underline{45}$, 637647

Honeck P, Nagele U, Michel MS (2008): Technische Innovationen in der endoskopischen Steintherapie. Urologe $\underline{47}, 587-590$

Huffman JL, Bagley DH, Schoenberg HW, Lyon ES (1983): Transurethral removal of large ureteral and renal pelvic calculi using ureteroscopic ultrasonic lithotripsy. J Urol $\underline{130}, 31-34$

Hussain M, Acher P, Penev B, Cynk M (2010): Redefining the limits of flexible ureterorenoscopy. J Endourol $\underline{25}$, 45-49

Knoll T, Alken P (2006): Ureterorenoskopie - gestern, heute und morgen. Urologe (Sonderheft) $\underline{45}, 185-186$

Knopfle E, Hamm M, Wartenberg S, Bohndorf K (2003): CT in ureterolithiasis with a radiation dose equal to intravenous urography: results in 209 patients. ROFO Fortschr Geb Rontgenstr Neuen Bildgeb Verfahr 175, 1667-1672

Krupp N, Bowman R, Tenggardjaja C, Jellison F, Hill B, Ebrahimi K, Smith J, Farley D, Baldwin DD (2010): Fluoroscopic organ and tissue-specific radiation exposure by sex and body mass index during ureteroscopy: editorial comment. J Endourol $\underline{24}$, 1067-1073 
Kumar P (2008): Radiation safety issues in fluoroscopy during percutaneous nephrolithotomy. Urol J $\underline{5}, 15-23$

Lahme S, Zimmermann V, Hochmuth A, Janitzki V (2008): Minimal-invasive PCNL (Miniperc). Urologe $\underline{47}, 563-568$

Lingeman JE, Newman DM, Siegel YI, Eichhorn T, Parr K (1995): Shock wave lithotripsy with the Dornier MFL 5000 lithotriptor using an external fixed rate signal. J Urol 154, 951-954

Lyon ES, Banno JJ, Schoenberg HW (1979): Transurethral ureteroscopy in men using juvenile cystoscopy equipment. J Urol $\underline{122}, 152-153$

Mancini JG, Raymundo EM, Lipkin M, Zilberman D, Yong D, Baňez LL, Miller MJ, Preminger GM, Ferrandino MN (2010): Factors affecting patient radiation exposure during percutaneous nephrolithotomy. J Urol 184, 2373-2377

Marshall VF (1963): Fiber optics in urology. J Urol $\underline{91}, 110-114$

Mobley TB, Myers DA, Grine WB, Jenkins JK, Jordan WR (1993): Low energy lithotripsy with the Lithostar: treatment results with 19962 renal and ureteral calculi. J Urol 149, 1419-1424

Nagele U, Knoll T, Schilling D, Michel MS, Stenzl A (2008a): Der untere Kelchstein. Urologe 47 , 875-884

Nagele U, Schilling D, Anastasiadis AG, Walcher U, Sievert KD, Merseburger AS, Kuczyk M, Stenzl A, (2008b): Minimal-invasive perkutane Nephrolitholapaxie (MIP) Urologe $\underline{47}, 1066-1073$

Obek C, Onal B, Kantay M, Yalcin V, Oner A, Solok V, Tansu N (2001): The efficiency of extracorporeal shock wave lithotripsy for isolated lower pole calculi compared with isolated middle and upper calceal calculi. J Urol 166, 2081-2084

Patlas M, Farkas A, Fischer D, Zaghal I, Hadas-Halpern I (2001): Ultrasound vs CT for the detection of ureteric stones in patients with renal colic. Br J Radiol $\underline{74}$, 901904 
Perez-Castro Ellendt E, Martinez-Pineiro JA (1980): Transurethral ureteroscopy. A current urology procedure. Arch Esp Urol $\underline{33}, 445-460$

Peschel R, Janetschek G, Bartsch G (1999): Extracorporeal shock wave lithotripsy vs ureteroscopy for distal calculi: a prospective randomized trial. J Urol $\underline{162}, 1909-1912$

Preminger GM, Tiselius HG, Assimos DG, Alken P, Buck C, Gallucci M, Knoll T, Lingeman JE, Nkada SY, Pearle MS (2007): Guideline for the management of ureteral calculi. J Urol $\underline{178}, 2418-2434$

Reuter MA: Entwicklung der Endourologie; in: Illustrierte Geschichte der Urologie; hrsg. v. Konert J, Dietrich HG unter Mitarbeit namhafter Autoren; Springer Verlag Berlin Heidelberg 2004, 139-194

Safak M, Olgar T, Bor D, Berkmen G, Gogus C (2009): Radiation doses of patients and urologists during percutaneous nepholithotomy. J Radiol. $\underline{29}$, 409-415

Salem HK (2009): A prospective randomized study comparing shock wave lithotripsy and semirigid ureteroscopy for the management of proximal ureteral calculi. Urology 74, 1221-1222

Sandilos P, Tsalafoutas I, Koutsokalis G, Georgious E, Yakoumakis E, Vlahos L (2006): Radiation doses to patients from extracorporeal shock wave lithotripsy. Health Phys $\underline{90}, 583-587$

Schmidt P, Pflüger H (2006): Extrakorporale Stoßwellenlithotrypsie (ESWL). Urologie Dezember 2005/Jänner 2006, 10-12

Schneider HJ (1982) What is an urolith and what is a recurent urolith? J Urol $\underline{127}, 72-$ 74

Schneider HJ (1985) Phytopharmaka in der Harnsteintherapie. Med Welt $\underline{36}, 1050-$ 1053 
Segura JW, Preminger GM, Assimos DG (1997): Ureteral stones clinical guidelines panel summary report on the management of ureteral calculi. J Urol $\underline{158}, 1915-1921$

Shannoun F, Blettner M, Schmidtberger H, Zeeb H (2008): Radiation protection in diagnostic radiology. Dtsch Ärztebl 105, 41-46

Strohmaier WL, Bartunek R (2008): Bildgebende Diagnostik - das Ende des AUG? Urologe $\underline{47}, 556-562$

Takagi T, Go T, Takayasu H, Aso Y (1971): Fiberoptic pyeloureteroscopy. Surgery. 70, $661-663$

Talati J, Khan S, Biyabani R, Khan RA, Naz I, Abbas F, Buchholz NP (2000): Reduction of radiation exposure to patients in the follow-up of skockwave lithotripsy. BJU Int $\underline{85}, 404-407$

Tamm EP, Silverman PM, Shuman WP (2003): Evaluation of the patient with flank pain and possible ureteral calculus. Radiology $\underline{228}$, 319-329

Tawfick ER (2010): Treatment of large proximal ureteral stones: extracorporeal shockwave lithotripsy versus semi-rigid ureteroscope with lithoclast. Int Arch $\underline{28}, 3-6$

Thomson JM, Glocer J, Abbott C, Maling TM, Mark S (2001): Computed tomography versus intravenous urography in diagnosis of acute flank pain from urolithiasis: a randomised study comparing imaging costs and radiation dose. Austr Radiol $\underline{45}, 291$ 297

Tiplitzky SI, Milhoua PM, Patel MB, Minsky L, Hoenig DM (2007): Case report: intrarenal arteriovenous fistula after ureteroscopic stone extraction with holmium laser lithotripsy. J Endourol 21, 530-532

Türk C, Knoll T, Köhrmann K.U (2008): Neue Leitlinien zur Harnsteintherapie. Urologe 47, 591-593

www.urologenportal.de/fileadmin/MDB/PDF/leitlinie-Harnsteine.pdf

S2-Leitlinien zur Diagnostik, Therapie und Metaphylaxe der Urolithiasis, Deutsche Gesellschaft für Urologie; Metabolische Harnsteinabklärung und Harnsteinmetaphylaxe, 4.7, 2009 
Danksagung

Mein ganz besonderer Dank gilt meinem Doktorvater, Prof. Dr. med. Gerhard M. Zöller, für die hervorragende Betreuung dieser Dissertation. Seine stete Ansprechbarkeit und vor allen Dingen seine fachliche und menschliche Kompetenz haben einen großen Beitrag zur Fertigstellung dieser Arbeit geleistet.

Zudem danke ich meinem Oberarztkollegen und Freund Dr. Holger Olfermann sowie Herrn Dr. Bernd Schulze-Brüggemann und meinem Mann Ulrich Theis für die Redigierung dieser Arbeit.

Ein „Dankeschön“ an die Mitarbeiter der urologischen Ambulanz für ihre Hilfe bei der Durchführung der ureterorenoskopischen Eingriffe und der Dokumentationen und an die Mitarbeiter der Urologischen Station für die Vorbereitung und Nachbetreuung der Patienten. 